\title{
UNIFIED BIJECTIONS FOR PLANAR HYPERMAPS WITH GENERAL CYCLE-LENGTH CONSTRAINTS
}

\author{
OLIVIER BERNARDI* AND ÉRIC FUSY ${ }^{\dagger}$
}

\begin{abstract}
We present a general bijective approach to planar hypermaps with two main results. First we obtain unified bijections for classes of maps or hypermaps defined by face-degree constraints and girth constraints. To any such class we associate bijectively a class of plane trees characterized by local constraints. This unifies and greatly generalizes several bijections for maps and hypermaps. Second, we present yet another level of generalization of the bijective approach by considering classes of maps with non-uniform girth constraints. More precisely, we consider well-charged maps, which are maps with an assignment of charges (real numbers) to vertices and faces, with the constraints that the length of any cycle of the map is at least equal to the sum of the charges of the vertices and faces enclosed by the cycle. We obtain a bijection between charged hypermaps and a class of plane trees characterized by local constraints.
\end{abstract}

\section{INTRODUCTION}

A planar map is an embedding of a connected planar graph in the sphere, considered up to orientation-preserving homeomorphism. A rich literature has been devoted to the enumerative combinatorics of planar maps by various approaches, such as Tutte's method 38 based on generating function equations, the matrix integral method initiated by Brézin et al. in [15, and the bijective approach initiated by Cori and Vauquelin [17] and popularized by Schaeffer [36].

Planar hypermaps are a natural generalization of planar maps. Precisely, a planar hypermap is a planar map in which faces are colored in two colors, say that there are dark faces and light faces, in such a way that every edge separates a light face from a dark face. The dark faces of the hypermap play the role of embedded hyperedges, and as such, hypermaps can be seen as embedded hypergraphs [16, and classical maps (embedded graphs) identify to hypermaps in which every edge has been replaced by a dark face of degree 2 ; see Figure 1(a).

Hypermaps have played a prominent role to tackle various problems: for instance an exact solution of the Ising model on random planar lattices has been obtained by a reduction to the enumeration of planar hypermaps with control on the face-degrees [8, 6]; and in a similar spirit different models of hard particles on random planar lattices have been exactly solved 8, 11. Hypermaps also encompass the notion of constellations, which are a convenient visual encoding of factorizations in the symmetric group [7, 25. In particular, the famous Hurwitz numbers 25, 22, 31, 18, (which count factorizations into transpositions, or equivalently certain branched coverings of the sphere) are naturally encoded by certain constellations. Bijective methods have played a crucial role in all these enumerative problems related to hypermaps.

In this article, we present a unified bijective approach for planar hypermaps. Our results generalize the bijective approach for maps presented in [4, 5] in two ways: first we deal with

Date: September 8, 2021.

*Department of Mathematics, Brandeis University, Waltham MA, USA, bernardi@brandeis.edu. Supported by NSF grant DMS-1308441 and DMS-1800681.

${ }^{\dagger}$ LIX, École Polytechnique, Palaiseau, France, fusy@lix.polytechnique.fr. Supported by the European project ExploreMaps (ERC StG 208471), the ANR grant "Cartaplus" 12-JS02-001-01, and the ANR grant "EGOS" 12-JS02-002-01. 


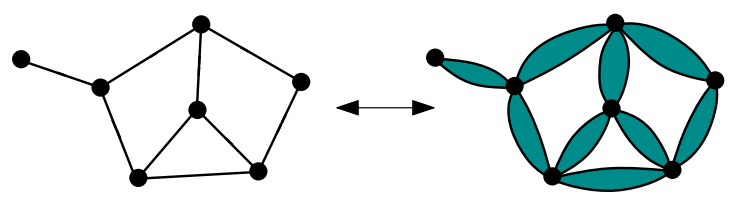

(a)

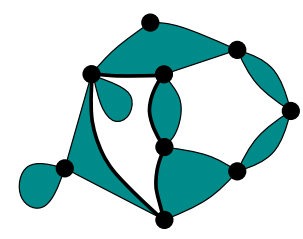

(b)

Figure 1. (a) A map and the corresponding hypermap (obtained by replacing every edge by a dark face of degree 2). (b) A general hypermap (with dark faces of arbitrary degrees), of ingirth 4 (due to the cycle indicated by bold lines).

the more general case of hypermaps, and second we consider more general cycle-length conditions via the new concept of charged maps. This approach also unifies and greatly generalizes several known bijections for hypermaps together with several known bijections for maps. We will discuss in details the relation between our approach and previously known bijections below (see Figure 3p and in Section 5. However, let us point out already that the bijections in $[8,10,11$ are recovered as special cases of our framework. These have applications to solving several statistical mechanics models on maps: Ising model, hard particle model, forest model, and blocked edge model. It is our hope that the toolbox we establish in the present article will find many more applications in the realm of statistical mechanics.

Our strategy is similar to the one developed in 4. Namely, we first establish a "master bijection" between a class of oriented hypermaps and a class of plane trees, that we call hypermobiles (see Figure 2(a) for an example). Then we specialize this master bijection to obtain our bijective results about classes of hypermaps defined in terms of face-degree conditions and girth conditions. This requires to exhibit canonical orientations characterizing these classes of maps, and then identifying the hypermobiles associated through the master bijection. To be precise, our canonical orientations and hypermobiles are actually weighted, that is, each edge carries a weight in $\mathbb{R}$; see Figure 2(a). In [4 we relied on the concept of minimal $\alpha$-orientations, that is, orientations such that the indegree at each vertex is fixed by a function $\alpha$, and containing no counterclockwise oriented cycle. We mention that Section 10.2 contains a generalizations of this framework to hypermaps which could be of independent interest.

In the first part of this article (Sections 35) we establish the master bijection and we use it to obtain bijections for classes of hypermaps defined by ingirth constraints. The ingirth for hypermaps is a generalization of the notion of girth for maps: it is defined as the smallest length of a cycle $C$ such that all faces adjacent to $C$ in the interior of $C$ are light (with the "interior" being defined with respect to a distinguished "outer face"). Similarly as in [5] (which deals with maps), we exhibit canonical orientations for hypermaps characterizing the ingirth constraints. Then, by applying the master bijection to canonically oriented (and weighted) hypermaps we obtain bijections between any class of hypermaps defined by face-degree constraints and ingirth constraints (with the sole restriction that the ingirth equals the degree of the outer face, which is dark), and a class of weighted hypermobiles (characterized by local degree and weight conditions). We show that the bijections for hypermaps in 17, 8, 10, 11 are special cases of our construction. In terms of counting, we obtain for any $d \geq 1$ an expression for the generating function of rooted hypermaps of ingirth $d$ and dark outer face of degree $d$, with control on the dark and light face degrees.

In a second part of the article (Sections 648), we consider charged hypermaps, which are a generalization of hypermaps well suited to study non-uniform cycle-length constraints. Roughly speaking, a fittingly charged hypermap is a hypermap together with an assignment of a real number, called charge, to each vertex and face such that 


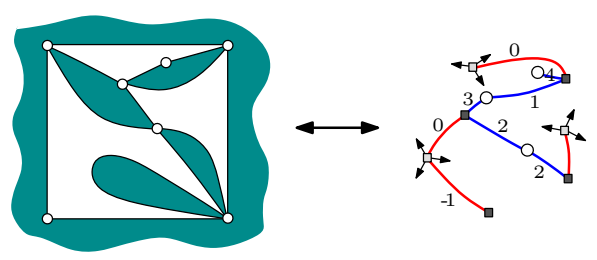

(a)

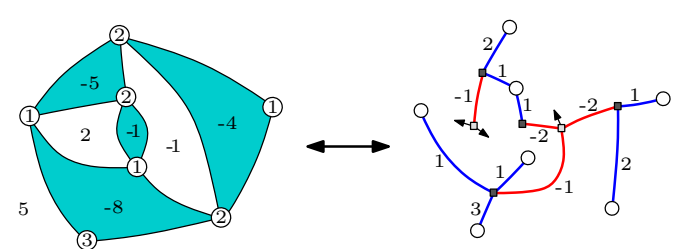

(b)

FiguRE 2. Left: example of correspondence between a planar hypermap of ingirth 4 and a (weighted) hypermobile. Right: example of correspondence between a charged hypermap and a (weighted) hypermobile.

- for any cycle $C$ enclosing a set $R$ of faces (possibly $R$ contains the outer face) such that $C$ is only incident to light faces of $R$, the sum of the charges of the vertices and faces enclosed by $R$ is at most the length of $C$,

- the charges of vertices are positive, and the sum of all charges is 0 .

See Figure 2(b) for an example of a fittingly charged hypermap, and Section 6 for more precise definitions. We show (again using the master bijection together with canonical orientations) that there is a bijection between the class of fittingly charged hypermaps and a class of weighted hypermobiles. This bijection keeps track of the face-degrees and of all the charges. An example is shown in the right-part of Figure 2, The bijections in the first part of the article are special cases of the bijection for charged hypermaps. We also show in Section 7 that the machinery of charged hypermaps can be used to get bijections for classes of annular hypermaps defined by face-degree conditions and two types of ingirth conditions (and we count these hypermaps in Section 8).

Let us mention that our master bijection comes in three "flavors" $\Phi_{+}, \Phi_{-}$and $\Phi_{0}$ (see Theorem 4). The flavor depends on the type of rooting of the hypermap: the hypermap has either a marked light face, a marked dark face or a marked vertex. Accordingly, our results for charged hypermaps come in three flavors (see Theorems 29, 30 and 31).

Charged hypermaps: a preview. The machinery of charged hypermaps proves well suited to establish unified bijections for hypermaps. We hope that this machinery will be useful to tackle new problems in the future, and in particular to prove isoperimetric inequalities for random maps in the spirit of [29, 28]. In order to give a preview of the notion of charged hypermaps, and illustrate its potential use, we now state a special case of our results about charged hypermaps. For simplicity, we will also restrict to the case of charged maps. Given a map $M$ with a distinguished root-vertex $v_{0}$, we call partial charge function a function $\sigma$ from the vertex set $V$ to $\mathbb{R}$. We say that $\sigma$ fits $M$ if the following conditions hold:

(a) for any subset $R$ of faces of $M$ defining a simply connected region of the sphere (after adding the edges and vertices incident only to faces in $R$ ), the set of edges $\partial R$ separating a face in $R$ and a face not in $R$ satisfies $|\partial R| \geq 2+\sum_{v}$ inside $R(\sigma(v)-2)$, with strict inequality if $v_{0}$ is inside $R$ (a vertex is said to be inside $R$ if all the incident faces are in $R$ ),

(b) $\sigma\left(v_{0}\right)=0, \sigma(v)>0$ for all $v \neq v_{0}$, and $\sum_{v \in V} \sigma(v)=2|V|-4$.

We call mobile a plane tree with two types of vertices - round and square - and with dangling half-edges - called buds - incident to square vertices. The excess of a mobile is the number of half-edges incident to round vertices minus the number of buds. We call suitably weighted a mobile with no edge joining two round vertices, where each edge joining a square vertex to a round vertex carries a positive weight, such that the sum of weights of edges incident to a square vertex $v$ is $\operatorname{deg}(v)-2$ (the weight is 0 for edges joining two square vertices). 
Theorem 1 (Special case of Theorem 31). There is a bijection between the set of pairs $(M, \sigma)$ where $M$ is a map with a distinguished root-vertex, and $\sigma$ is a partial charge function fitting $M$, and the set of suitably weighted mobiles of excess 0. Moreover, faces of degree $k$ of the map correspond bijectively to square vertices of degree $k$ in the mobile, and vertices of charge $w$ correspond bijectively to round vertices of weight $w$ (i.e., the incident edge weights sum to $w)$.

We hope that this type of bijections can be used to study cycle lengths in large random maps, and their scaling limit, the so-called Brownian map [26, 27, 30. In particular, since typical distances in random maps with $n$ edges scale like $n^{1 / 4}$, it would be interesting to look at a partial charge function $\sigma$ such that $\sigma(v)=2 \pm \frac{\alpha}{n^{1 / 4}}$ for all $v$ (for some constant $\alpha$, and with the signs being independent and uniformly random). In this case, Theorem 1 gives a way of counting maps such that the boundary of any simply connected set of faces $R$ satisfies $|\partial R| \geq 2+\sum_{v}$ inside $R=(v)-2$, which is asymptotically Gaussian of amplitude $\alpha \sqrt{\beta} n^{1 / 4}$ if $R$ contains $\beta n$ vertices. This may give a bijective method for proving isoperimetric inequalities in the spirit of [28].

Relation with other bijections for maps and hypermaps. As already said, the present article generalizes our previous work on maps (again this relies on the fact that maps are merely hypermaps with all dark faces of degree 2). The diagram in Figure 3 summarizes the relations between the bijections in the present article and previous ones. Precisely, the master bijection for hypermaps given in Section 2 generalizes the master bijection for maps given in 4, and the bijection for hypermaps of ingirth $d$, dark outer face of degree $d \geq 1$ and control on the face-degrees, generalizes the bijection for plane maps of outer degree $d$ and girth $d$ obtained in [5]. The case $d=1$ for hypermaps identifies to the bijection of Bousquet-Mélou and Schaeffer [8] (stated in terms of bipartite maps in [8]) with applications to the Ising model and the hard particle model. The case $d \geq 2$ admits a natural specialization to $d$-constellations, which coincides with the bijection of BousquetMélou and Schaeffer [7]. And we also provide a special formulation for the case $d=0$, from which we recover the bijection by Bouttier, Di Francesco and Guitter for vertex-rooted hypermaps [10] and for vertex-rooted hypermaps with blocked edges [1] (with applications to hard particle models, the Ising model, and forested maps enumeration).

Moreover, since we generalize the results for maps in [5], we also recover the various known bijections for maps obtained as specializations in [5]: in particular the case $d=1$ in [5] identifies to the bijection of Bouttier et al. in [9], the case $d=2$ includes the bijections of [35] for bipartite maps and of [32] for loopless triangulations, the case $d=3$ includes the bijection of $[21$ for simple triangulations, and the case $d=4$ includes the bijection of 36 . Sect. 2.3.3] for simple quadrangulations. Similarly the bijection for annular hypermaps (two marked faces) in Section 7 generalizes the bijection for annular maps obtained in [5. Sect. 5].

In contrast, the results in the second part of the article (bijection between hypermobiles and charged hypermaps, allowing to formulate non-uniform girth constraints) are totally new (the subcase of charged maps is not covered in [5, and in fact dealing directly with the more general case of charged hypermaps somehow simplifies the proofs).

We would like to mention two other general combinatorial methods for counting maps. Recall that our master bijection for hypermaps generalizes the master bijection for maps given in [4. In the recent article [1, Albenque and Poulalhon have presented another general bijective approach to maps. The two approaches are closely related and use essentially the same canonical orientations (exhibited in 44). The main difference between the approach in [4] and in [1] is that the master bijections between oriented maps and trees are different (one tree is a spanning tree of the map, while the other is a spanning tree of the quadrangulation of the map). Both master bijections are actually based on the two types of trees shown to be associated with "minimal accessible orientations" in the article 2] (which has been 


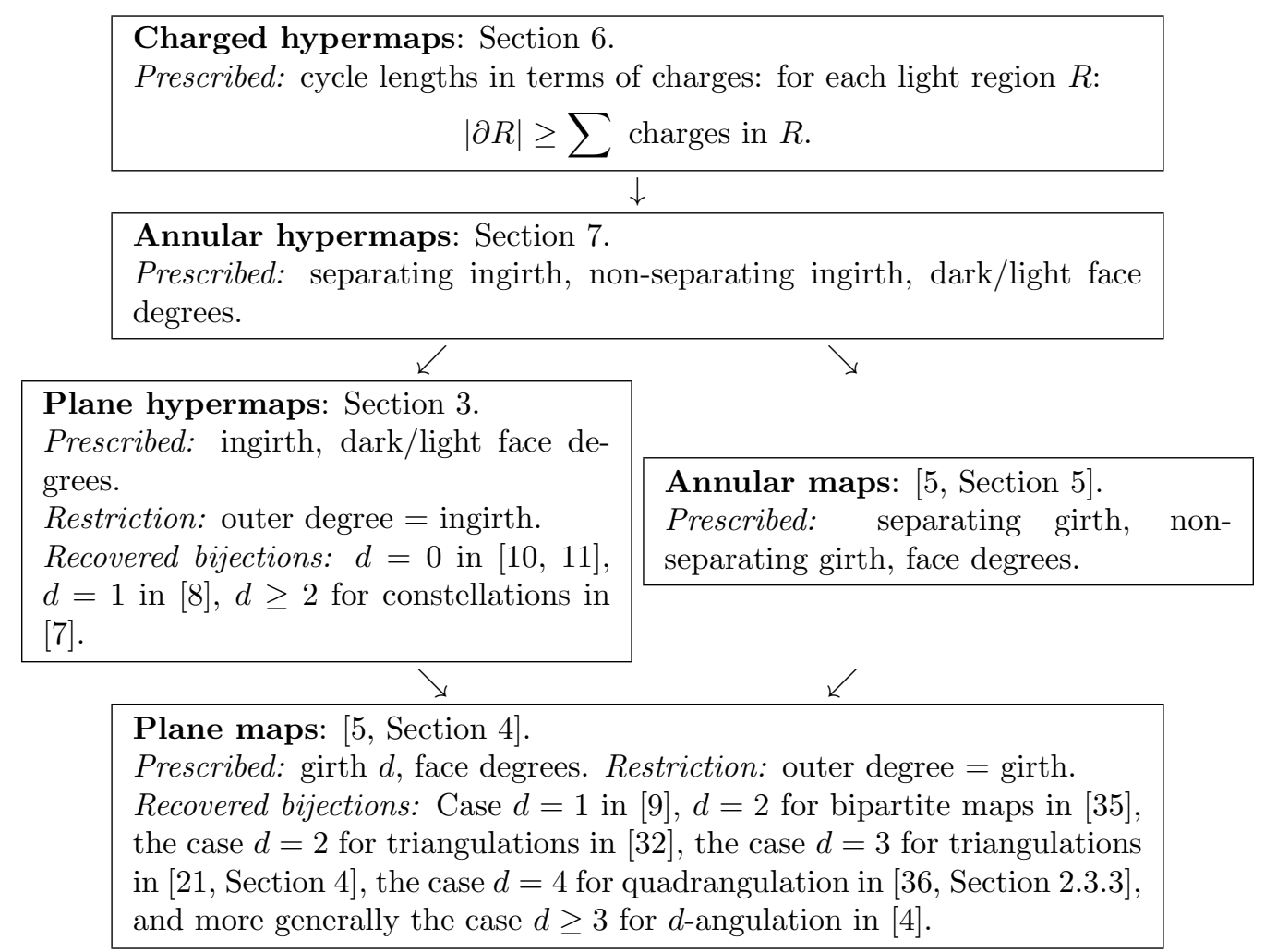

FiguRE 3. Relation between the bijections in this article and previous ones; arrows indicate specializations.

reformulated and extended to higher genus in [3]). The existence of these two "master bijections" explains why two different bijections have been found for several classes of maps, one being generalized in $[4$ and the other in [1. For instance, , 4] and 1] respectively generalize the bijections originally found in 21 and 33 for simple triangulations (i.e. triangulations of girth 3). It seems however that the master bijection in [4] is better suited to deal with classes of maps where several face degrees are allowed.

Another unified combinatorial approach to maps was developed by Bouttier and Guitter in [13] (building on [12]). They show that one of the desirable feature of trees, namely that they are easy to enumerate thanks to their natural recursive structure, could be directly achieved at the level of the maps themselves via so-called slice decomposition of maps. With this method, they obtain the generating function of maps of pseudo girth $d$ (maps in which cycles have length at least $d$, except for the contours of faces, which can be of length $d-1$ ) with control on the face-degrees, thereby generalizing the counting results of [4] (in which faces of degree $d-1$ were forbidden).

It is unclear if the methods used in 1, 13 can be generalized to hypermaps, and/or to charged maps.

Outline. The outline of the paper is as follows. In Section 2, we define hypermaps and hypermobiles, and we present the master bijection between a class of oriented hypermaps and a class of hypermobiles. In Section 3, we consider for each $d \geq 1$ the class $\mathcal{C}_{d}$ of hypermaps of ingirth $d$ with a dark outer face of degree $d$. By applying the master bijection to canonically oriented maps in $\mathcal{C}_{d}$ we obtain a bijection between $\mathcal{C}_{d}$ and a class of hypermobiles. In Section 4, we obtain the generating function of the class $\mathcal{C}_{d}$ of hypermaps counted according to the degree distribution of their faces (by recursively decomposing the associated hypermobiles). In Section 5 , we show that the bijections described in [7, 8, 10, 11] are special 
cases of the bijections obtained in Section 3. In Section 6, we obtain a general bijection for fittingly charged hypermaps. As before, this bijection is obtained by first characterizing fittingly charged hypermaps by suitable canonical orientations and then applying the master bijection. In Section 7, we use the framework of charged hypermaps to obtain bijections for classes of annular hypermaps characterized by separating and non separating girth constraint. In Section 8 we obtain the generating function of those classes. In Section 9 , we gather some proofs about the master bijection. In Section 10, we gather our proofs about canonical orientations.

\section{MASTER BIJECTION FOR HYPERMAPS}

2.1. Hypermaps and hyperorientations. A map is a connected graph embedded on the sphere, considered up to continuous deformation. An Eulerian map is a map such that all vertices have even degree. Such maps are also those whose faces can be bicolored - say there are dark faces and light faces - in such a way that every edge separates a dark face from a light face. Note that this bicoloration is unique up to the choice of the color of a given face. An hypermap is a face-bicolored Eulerian map; dark faces are also called hyperedges. The underlying map is the (Eulerian) map obtained from the hypermap by forgetting the face types. A corner of a map is the an angular section between two consecutive half-edges around a vertex. The degree of a vertex or face $a$, denoted by $\operatorname{deg}(a)$, is the number of incident corners.

A face-rooted hypermap is a hypermap with a marked face (either dark or light) called the outer face. The other faces are called inner faces. The vertices and edges are called outer if they are incident to the outer face and inner otherwise. The outer degree of a facerooted hypermap is the degree of the outer face. Observe that face-rooted hypermaps, can also be thought of as hypermaps embedded in the plane (with the outer face being infinite), and for this reason they are sometimes called plane hypermaps. A dark-rooted (resp. lightrooted) hypermap is a face-rooted hypermap such that the outer face is dark (resp. light). A vertex-rooted hypermap is a hypermap with a marked vertex called the root-vertex. A corner-rooted hypermap is a hypermap with a marked corner called the root-corner.
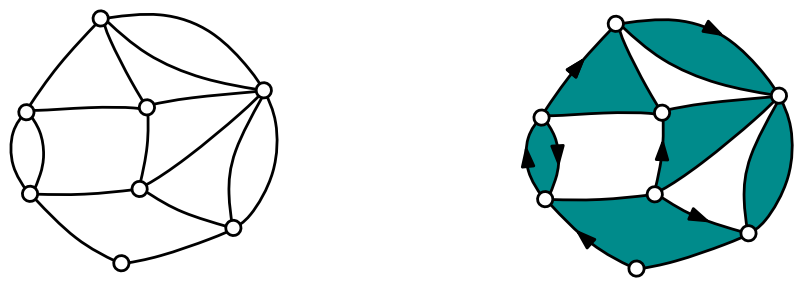

FiguRE 4. Left: an Eulerian map $M$ (all vertices of $M$ have even degree). Right: a hypermap (having $M$ as underlying Eulerian map) endowed with a hyperorientation.

A hyperorientation $O$ of a hypermap $H$ is a partial orientation (edges are either oriented or unoriented) of the edges of $H$ such that each oriented edge has a dark face on its right. Oriented edges are called 1-way, unoriented edges are called 0-way. Directed outer edges are called cw-outer or ccw-outer respectively, depending on whether they have the outer face on their left or on their right. A directed path from $u$ to $v$ is a sequence of 1-way edges $e_{1}, \ldots, e_{k}$ such that the origin of $e_{1}$ is $u$, the end of $e_{k}$ is $v$, and for all $i \in\{1, \ldots, k-1\}$ the end of $e_{i}$ is the origin of $e_{i+1}$. This directed path is a circuit if $u=v$. A circuit is called simple if the origins of $e_{1}, \ldots, e_{k}$ are all distinct. If $H$ is an hyperoriented face-rooted hypermap, a simple circuit $C$ is called clockwise if the outer face is in the region delimited by $C$ on the left of $C$, and counterclockwise otherwise. Similarly, if $H$ is a vertex-rooted hypermap, a simple circuit $C$ is said to be clockwise if the root-vertex is either on $C$ or 
in the region delimited by $C$ on the left of $C$; and $C$ is said to be counterclockwise if the root-vertex is either on $C$ or in the region delimited by $C$ on the right of $C$ (note that a circuit passing by the root-vertex is clockwise and counterclockwise at the same time). The hyperorientation is called minimal if it has no counterclockwise circuit, and is called accessible from a vertex $v$ if every vertex $u$ can be reached from $v$ by a directed path. By a slight abuse of terminology, we will often refer to a hyperoriented hypermap as a hyperorientation.

We now define three families of hyperorientions that will play a central role in the master bijections (see Figure 5). We call face-rooted hyperorientation a face-rooted hypermap endowed with a hyperorientation. Light-rooted, dark-rooted and vertex-rooted hyperorientations are defined similarly.

- We define $\mathcal{H}_{+}$as the family of light-rooted hyperorientations that are accessible from every outer vertex, minimal, and such that every outer edge is 1-way (the outer face contour is a clockwise circuit, not necessarily a simple circuit).

- We define $\mathcal{H}_{-}$as the family of dark-rooted hyperorientations that are accessible from every outer vertex, such that the outer face contour is a simple counterclockwise circuit, and it is the unique counterclockwise circuit in the hyperorientation.

- We define $\mathcal{H}_{0}$ as the family of vertex-rooted hyperorientations that are accessible from the root vertex $v_{0}$, and minimal.
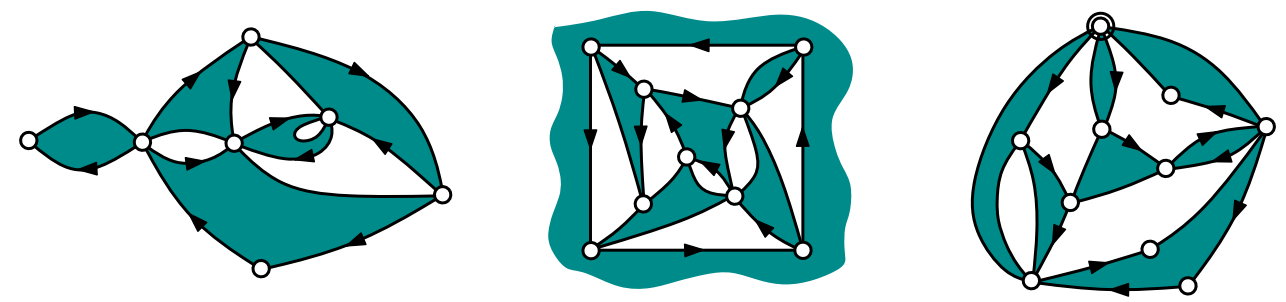

FiguRE 5. Left: a (light-rooted) hyperorientation in $\mathcal{H}_{+}$. Middle: a (darkrooted) hyperorientation in $\mathcal{H}_{-}$. Right: a (vertex-rooted) hyperorientation in $\mathcal{H}_{0}$.

Remark 2. We point out that if a hyperorientation $H$ is in $\mathcal{H}_{-}$, then there is no inner edge of $H$ incident to an outer-vertex and oriented 1-way toward that outer vertex. Indeed, if we suppose by contradiction that such an inner edge $e$ exists, then because $H$ is accessible, there is a path $P$ of inner edges starting at an outer vertex and ending with the edge $e$. However, this path $P$ together with the contour of the outer face creates a counterclockwise cycle; see Figure 6. This gives a contradiction. Similarly, if a hyperorientation is in $\mathcal{H}_{0}$, then every incidence of an edge $e$ with the root-vertex $v_{0}$ is such that $e$ is 0 -way or 1-way out of $v_{0}$.

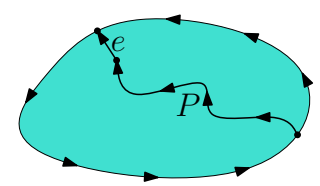

Figure 6 . The directed path $P$ of inner edges starting at an outer vertex and ending with the edge $e$. 
2.2. Master bijection $\Phi_{*}$. We now define the classes of planes trees which are in bijection with the classes of hyperorientations in $\mathcal{H}_{+}, \mathcal{H}_{-}$and $\mathcal{H}_{0}$. We consider plane trees with dangling half-edges called buds. An hypermobile is a plane tree with buds having 3 types of vertices - round, dark square, and light square - and such that

- buds are incident to light square vertices,

- every edge is incident to exactly one dark square vertex (hence the edge joins a dark square vertex to either a light square vertex or a round vertex).

The degree of a vertex in the hypermobile is the number of incident half-edges (including buds, for light square vertices). The excess of the hypermobile is the number of edges with a round extremity, minus the number of buds. We denote respectively by $\mathcal{T}_{+}, \mathcal{T}_{-}$, and $\mathcal{T}_{0}$ the families of hypermobiles of positive excess, negative excess, and zero excess.

We now describe the master bijection for hypermaps. Actually, there are 3 bijections denoted by $\Phi_{+}, \Phi_{-}$and $\Phi_{0}$, and mapping the classes of hyperorientations $\mathcal{H}_{+}, \mathcal{H}_{-}, \mathcal{H}_{0}$ respectively to the classes of hypermobiles having positive, negative, and zero excess.

Let $X$ be an hyperorientation in $\mathcal{H}_{*}$ with $* \in\{+,-, 0\}$. The hypermobile $\Phi_{*}(X)$ is obtained as follows:

- Place a dark (resp. light) square vertex of $\Phi_{*}(X)$ in each dark (resp. light) face of $X$; the vertices of $X$ will become the round vertices of $\Phi_{*}(X)$.

- Create the edges of $\Phi_{*}(X)$ by applying to each edge of $X$ the local rule indicated in Figure 7 (ignore the weights $w$ for the time being). Then erase all the edges of $X$.

- To complete the construction in the case $*=+$ delete the light square vertex in the outer face of $X$ (together with the incident buds). To complete the construction in the case $*=-$, delete the dark square vertex in the outer face of $X$, all the outer vertices of $X$ and the edges linking them. To complete the construction in the case $*=0$, simply delete the root-vertex of $X$.

The mappings $\Phi_{*}$, are illustrated in Figure 8 .

\begin{tabular}{|c|c|c|}
\hline & 1-way edge & 0 -way edge \\
\hline \multicolumn{3}{|l|}{ In the hypermap } \\
\hline & $w$ & $w$ \\
\hline In the hypermobile & & $w \quad$ \\
\hline & 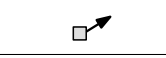 & 口 \\
\hline
\end{tabular}

Figure 7. Local rules applied in the bijections $\Phi_{+}, \Phi_{-}, \Phi_{0}$ to every edge of a hyperorientation. The rule for the transfer of a weight $w$ is also indicated (for the edge-weighted version of the bijections).

Remark 3. For $X \in \mathcal{H}_{+}$, all the outer edges are oriented 1-way with the root-face on their left, hence the local rules of Figure 7 do not create any edge incident to the light square vertex in the outer face of $X$ (only buds). Thus, the last step to complete $\Phi_{+}(X)$ only deletes an isolated vertex. Similarly, for $X \in \mathcal{H}_{0}$, the last step to complete $\Phi_{0}(X)$ only deletes an isolated vertex. Lastly, for $X \in \mathcal{H}_{-}$the local rules of Figure 7 do not create any edge incident to the outer vertices of $X$, except for the edges joining them to the dark square vertex in the outer face of $X$ (because by Remark 2 no inner edge is 1-way toward an outer vertex). Hence the last step to complete $\Phi_{-}(X)$ only deletes an isolated "star graph" made of these vertices and edges.

Theorem 4. For $* \in\{+,-, 0\}$ the mapping $\Phi_{*}$ is a bijection between $\mathcal{H}_{*}$ and $\mathcal{T}_{*}$. For $\Phi_{+}$ the outer degree of $\gamma \in \mathcal{H}_{+}$is equal to the excess of $\tau=\Phi_{+}(\gamma)$, for $\Phi_{-}$the outer degree of $\gamma \in \mathcal{H}_{-}$is equal to minus the excess of $\tau=\Phi_{-}(\gamma)$. 


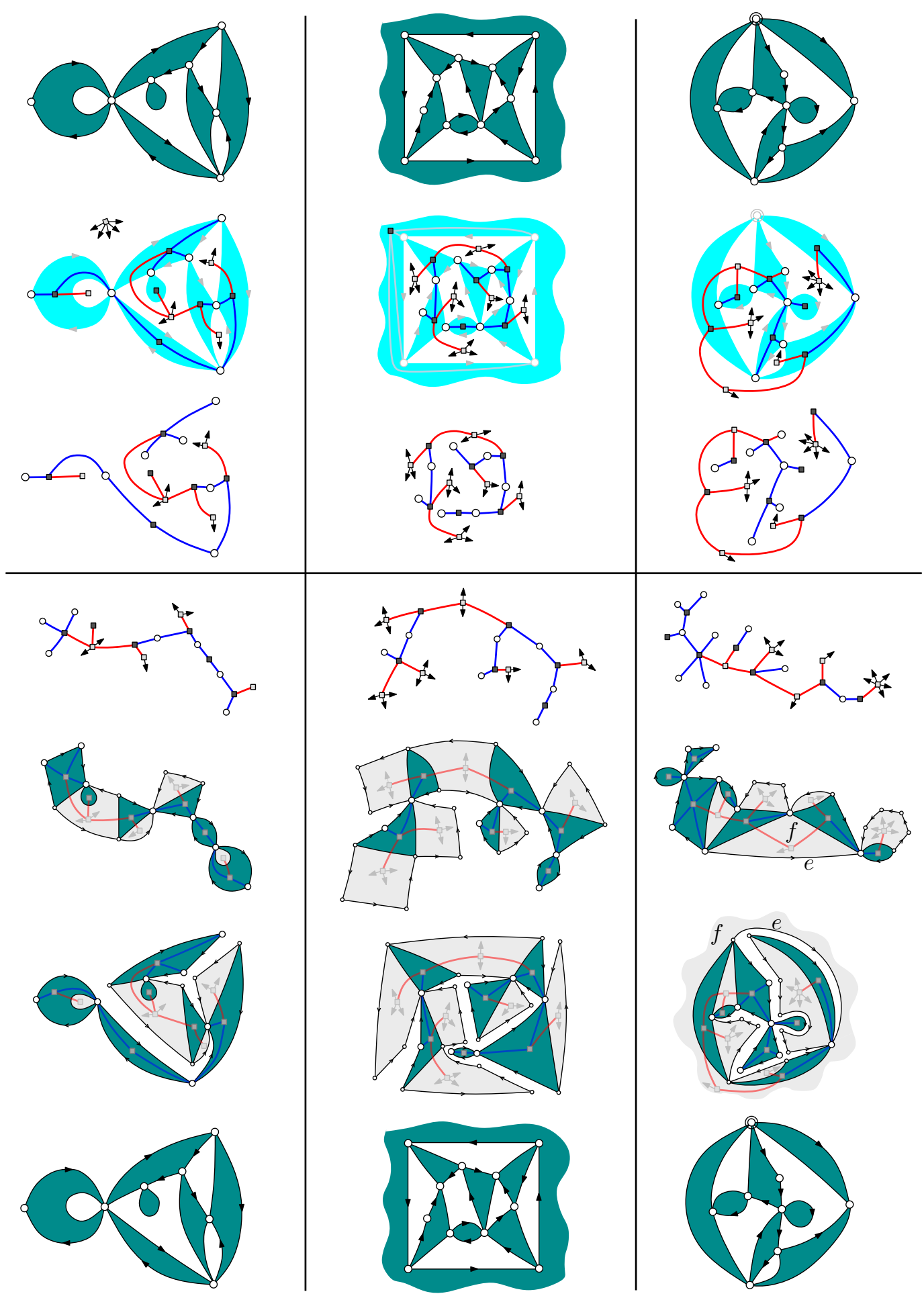

Figure 8 . The master bijection $\Phi_{*}$ from hyperorientations to hypermobiles (upper part: left $\Phi_{+}$, middle $\Phi_{-}$, right $\Phi_{0}$ ) and its inverse $\Psi_{*}$ (lower part: left $\Psi_{+}$, middle $\Psi_{-}$, right $\Psi_{0}$ ). Hypermobile edges are blue or red whether they have a round extremity or not. 
The proof of Theorem 4 is postponed to Section 9. We will now formulate a version of the bijections $\Phi_{*}$ for edge-weighted hyperorientations, and explain the parameter correspondences.

A hyperorientation is weighted by assigning a weight in $\mathbb{R}$ to each edge. In that case, the weight of a vertex is the total weight of its incident ingoing edges, the weight of a light face is the total weight of its incident 0-way edges, and the weight of a dark face is the total weight of its incident edges. For hyperorientations is in $\mathcal{H}_{-}$, we take the convention that all outer edges (which are 1-way) have weight 1. Similarly a hypermobile is weighted by assigning a weight in $\mathbb{R}$ to each of its (non-bud) edges. The weight of a vertex of a hypermobile $M$ is the total weight of its incident (non-bud) edges, and the degree of a vertex of $M$ is the number of incident half-edges (including buds, for light square vertices). The local rule of Figure 7 can directly be adapted so as to transfer the weight of an edge of the hypermap to the corresponding edge in the associated hypermobile, see Figure 7 Hence, Theorem 4 has the following corollary.

Corollary 5. The mapping $\Phi_{+}$(resp. $\left.\Phi_{-}, \Phi_{0}\right)$ is a bijection between weighted hyperorientations from $\mathcal{H}_{+}$(resp. $\mathcal{H}_{-}, \mathcal{H}_{0}$ ) and weighted hypermobiles of positive excess (resp. negative excess, zero excess).

We now formulate the parameter correspondences between hypermaps and hypermobiles. In order to make a formulation valid simultaneously for $\Phi_{+}, \Phi_{-}$and $\Phi_{0}$, we first define the frozen vertices, edges and faces of a hyperorientation $H$ in $\mathcal{H}_{+}, \mathcal{H}_{-}$and $\mathcal{H}_{0}$. For $H \in \mathcal{H}_{+}$, only the outer face is frozen. For $H \in \mathcal{H}_{0}$, only the root-vertex is frozen. For $H \in \mathcal{H}_{-}$, the outer face, all the outer edges and all the outer vertices are frozen. With this terminology, for $* \in\{+,-, 0\}$, for $H \in \mathcal{H}_{*}$ and $T=\Phi_{*}(X)$, we have

- each non-frozen light (resp. dark) face of $H$ corresponds to a light (resp. dark) square vertex of the same degree and same weight in $T$;

- each non-frozen edge of $H$ corresponds to a (non-bud) edge of the same weight in $T$;

- each non-frozen vertex of $H$ of weight $w$ and indegree $\delta$ corresponds to a round vertex of $T$ of weight $w$ and degree $\delta$.

2.3. Inverse bijections $\Psi_{*}$. We will now describe the inverses $\Psi_{+}, \Psi_{-}$, and $\Psi_{0}$ of the bijections $\Phi_{+}, \Phi_{-}$, and $\Phi_{0}$. Let $T$ be a hypermobile. We associate with $T$ an outerplanar map $\widehat{T}$ (a plane map such that every vertex is incident to the outer face) as follows:

- for each dark (resp. light) vertex of degree $d$ in $T$ we create a dark (resp. light) polygon of degree $d$ following the rules illustrated in Figure 9

- for each edge $e$ of $T$ between a dark square and a light square vertex, we glue together the two face sides of the corresponding polygons at $e$;

- for each round vertex $v$ of $T$ of degree $d$ we merge the $d$ neighboring polygon corners with the vertex $v$.
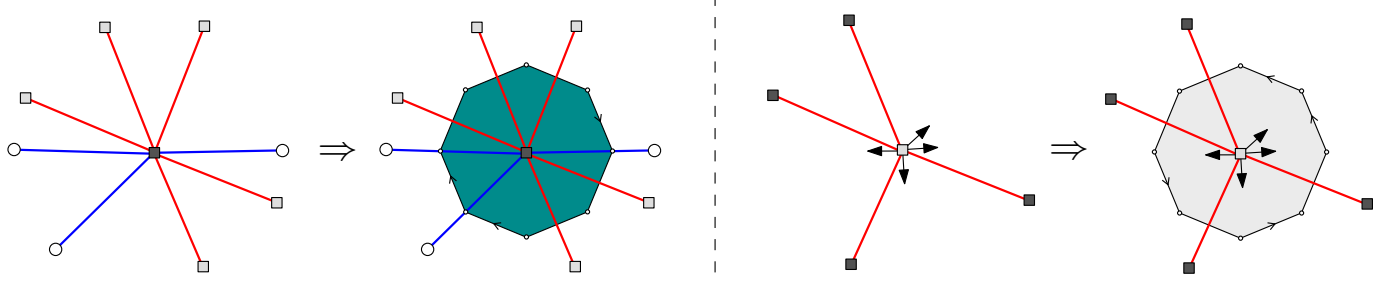

FiguRE 9. Left: growing a dark polygon at a dark square vertex of a hypermobile. Right: growing a light polygon at a light square vertex of a hypermobile. 
See Figure 10 for an example. Note that the inner edges of the outerplanar map $\widehat{T}$ are 0 -way and the outer edges are 1-way: cw-outer edges of $\widehat{T}$ correspond to edges between a round and a dark square vertex in $T$, ccw-outer edges of $\widehat{T}$ correspond to buds of $T$.

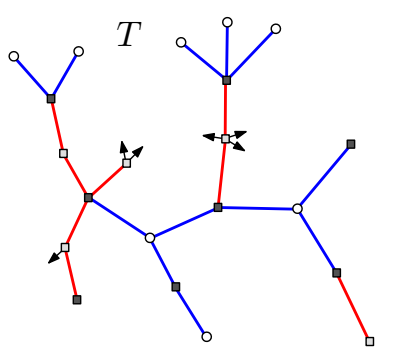

(a)

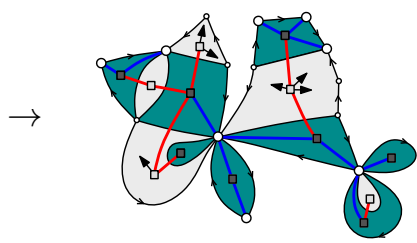

(c)

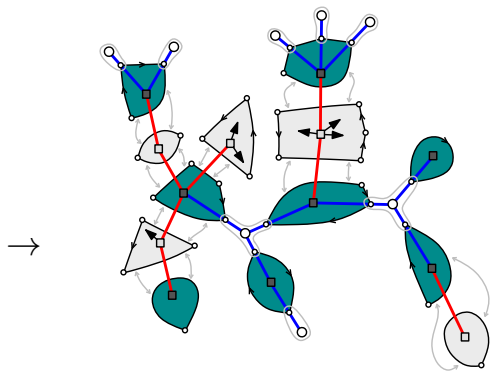

(b)

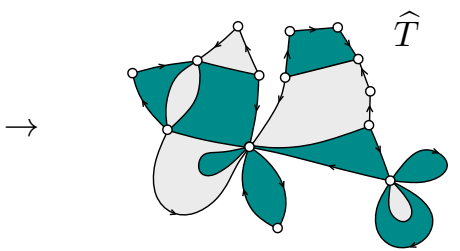

(d)

FIGURE 10. From a hypermobile $T$ to the associated outerplanar map $\widehat{T}$. (a) The hypermobile $T$. (b) Creating the polygons around square vertices. (c) Gluing polygon-sides corresponding to each edge of $T$ between a dark square and a light square vertex, and merging polygon-corners neighboring each round vertex of $T$. (d) The outerplanar map $\widehat{T}$.

The mappings $\Psi_{+}, \Psi_{-}, \Psi_{0}$ (which will be proved to be the inverse bijections of $\Phi_{+}, \Phi_{-}$, $\Phi_{0}$ respectively) are defined as follows. Let $T$ be a hypermobile, and let $\widehat{T}$ be the associated outerplanar map. We will now define a canonical way of gluing together the cw-outer and ccw-outer edges of $\widehat{T}$; see Figure 11

A word $w$ (i.e. sequence of letters) on the alphabet $\{a, \bar{a}\}$ is a parenthesis word if $w$ has as many letters $a$ as letters $\bar{a}$, and for any prefix of $w$ the number of letters $a$ is at least equal to the number of letters $\bar{a}$. A cyclic word is a word considered up to cyclic shift of the letters. Given a cyclic word $w$ on the alphabet $\{a, \bar{a}\}$, we say that a letter $a$ and a letter $\bar{a}$ are $c w$-matching, if the subword of $w$ starting after the letter $a$ and ending before the letter $\bar{a}$ is a (possibly empty) parenthesis word. An example is given in Figure 11(a). It is easy to see that for any letter $a$ there is at most one cw-matching letter $\bar{a}$ and vice-versa. Moreover if a cyclic word $w$ has $n_{a}$ letters and $n_{\bar{a}}$ letters $\bar{a}$ with $n_{a} \geq n_{\bar{a}}\left(\right.$ resp. $n_{a} \leq n_{\bar{a}}$ ), then all the letters are cw-matching except for $n_{a}-n_{\bar{a}}$ letters $a$ (resp. $n_{\bar{a}}-n_{a}$ letters $\bar{a}$ ).

We are now ready to define a canonical way of gluing the cw-outer and ccw-outer edges of $\widehat{T}$. We associate a cyclic word $w_{T}$ with the sequence of outer edges appearing in clockwise order around the outer face of $\widehat{T}$ by encoding the cw-outer and ccw-outer edges by the letters $a$ and $\bar{a}$ respectively. We say that a cw-outer edge and a ccw-outer edge of $\widehat{T}$ are $c w$-matching if the corresponding letters $a$ and $\bar{a}$ are cw-matching in $w_{T}$. It is easy to see that all the pairs of cw-matching edges can be glued together into 1-way edges (that is, there is no breach of planarity in doing so for every pair of cw-matching edges). An example is given in Figure 11(b). If the excess $\epsilon$ of $T$ is positive, then $\widehat{T}$ has $\epsilon$ more cw-outer edges than ccw-outer edges. Thus the map obtained after gluing the cw-matching edges of $\widehat{T}$ has 
an outer face of degree $\epsilon$ which is incident only to cw-outer edges. Hence coloring the outer face as light gives an oriented light-rooted hypermap, that we denote by $\Psi_{+}(T)$. Similarly, if the excess $\epsilon$ of $T$ is negative, then the map obtained after gluing the cw-matching edges of $\widehat{T}$ has an outer face of degree $-\epsilon$ which is incident only to ccw-outer edges. Hence coloring the outer face as dark gives an oriented dark-rooted hypermap, that we denote $\Psi_{-}(T)$. Lastly, if the excess of $T$ is 0 , then all the outer edges of $\widehat{T}$ are glued. Moreover, there is a unique vertex $v_{0}$ of the glued map without incident ingoing edges. In this case, taking $v_{0}$ as the root-vertex gives an oriented vertex-rooted hypermap that we denote by $\Psi_{0}(T)$. Examples for $\Phi_{+}, \Phi_{-}, \Phi_{0}$ are given in Figure 8

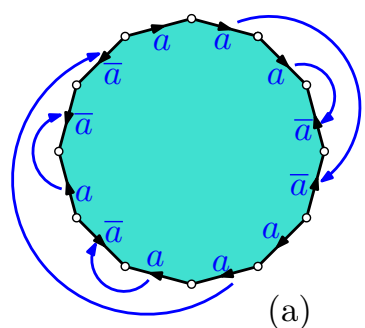

(a)

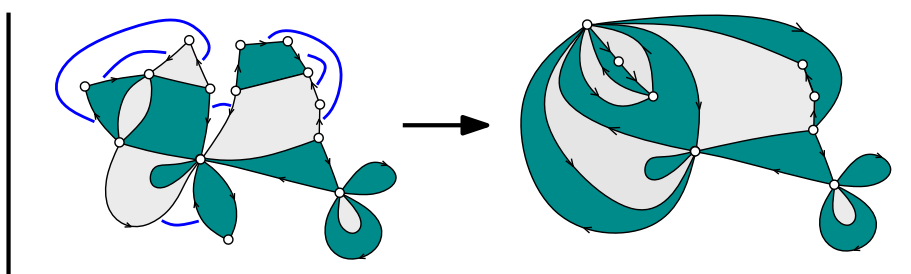

(b)

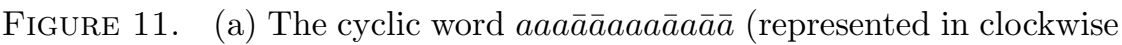
order around a polygon), and the cw-matching pairs of $a$ 's and $\bar{a}$ 's (indicated by the arrows). (b) Gluing of the cw-matching pairs of edges of the outerplanar maps $\widehat{T}$.

Theorem 6. The mappings $\Psi_{+}, \Psi_{-}$, and $\Psi_{0}$ are the inverses of the bijections $\Phi_{+}, \Phi_{-}$, and $\Phi_{0}$ respectively.

We shall prove Theorem 6 in Section 9

2.4. Alternative formulation of the inverse bijections $\Psi_{*}$. For the sake of completeness, we now give an alternative description of the mappings $\Psi_{*}$, which is closer to the description of many known bijections (in particular, the bijections in [7, 8, 11] discussed in Section 5). Let $T$ be a hypermobile, where buds are interpreted as outgoing sprouts. Add ingoing sprouts as follows: for each edge $e=\{u, v\} \in T$ connecting a round vertex $u$ to a dark square vertex $v$, insert an ingoing sprout in the corner following $e$ in counterclockwise order around $v$. See Figure 12 for an example. We associate a cyclic word $w_{T}$ with the sequence of sprouts appearing in clockwise order around the outer face of $T$ (with the outer face on the left of the walker) by encoding the ingoing and outgoing sprouts by the letters $a$ and $\bar{a}$ respectively. We join the cw-matching ingoing and outgoing sprouts to form oriented edges, and then remove from $T$ the round vertices and their incident edges. The embedded partially oriented graph $G$ thus obtained is called the partial closure of $T$. If $T$ has nonzero excess $\epsilon$, then there remain $|\epsilon|$ unmatched sprouts in $G$ (which are ingoing if $\epsilon>0$, outgoing if $\epsilon<0)$. The complete closure of $T$, denoted by $G^{\prime}$ is defined as follows: if $\epsilon=0$ then $G^{\prime}=G$, while if $\epsilon>0$ (resp. $\epsilon<0$ ) $G^{\prime}$ is obtained from $G$ by adding a new light (resp. dark) square vertex $v_{0}$ in the face containing all the sprouts and connecting these sprouts to $v_{0}$ by new edges directed away from $v_{0}$ (resp. toward $v_{0}$ ). Observe that $G^{\prime}$ is a bipartite map since every edge is incident to one dark square vertex. Finally, we call $\Psi_{*}(T)$ (for $* \in\{+,-, 0\}$ depending on whether the excess $\epsilon$ is positive, negative, or zero) the dual of $G^{\prime}$ which is a hypermap (the dual of dark squares are taken to be dark faces). The edges of $\Psi_{*}(T)$ are oriented as follows: an edge $e^{\prime}$ of $\Psi_{*}(T)$ which is dual to an oriented edge $e$ of $G^{\prime}$ (made by connecting two sprouts) is oriented 1-way from the right-side of $e$ to the left-side of $e$, while an edge of $\Psi_{*}(T)$ which is dual to an original edge of $T$ is left unoriented. Lastly, if the excess $\epsilon$ is non-zero we take the dual of the vertex $v_{0}$ of $G^{\prime}$ to be the root-face of 


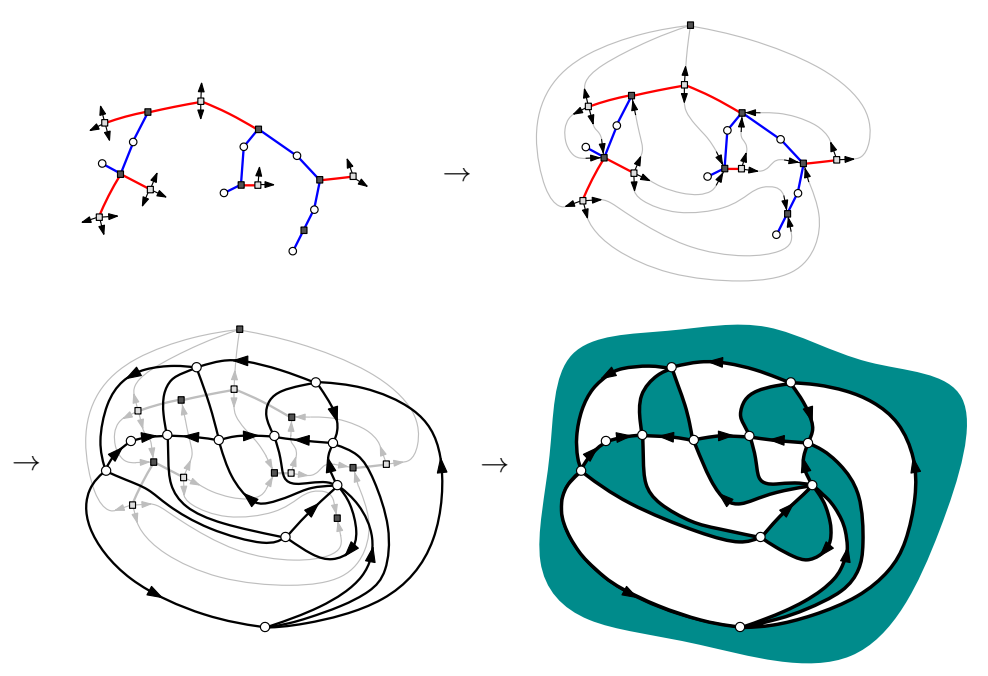

Figure 12. The closure mapping $\Psi_{-}$performed by joining pairs of $\mathrm{cw}^{-}$ matching outgoing and ingoing sprouts, and then taking the dual.

$\Psi_{*}(T)$, while if $\epsilon=0$ we take the dual of the root-face of $G^{\prime}$ to be the root-vertex of $\Psi_{0}(T)$. See Figure 12 for an example.

It is easy to see that the formulation with sprouts given here is equivalent to the formulation with outerplanar maps given above. Indeed, if we superimpose the hypermobile with the associated outerplanar map, then each cw-matching operation in one formulation is equivalent to a cw-matching operation in the other formulation.

2.5. Relation with the master bijection for maps defined in 5. In a preceding article 4] we gave master bijections for planar maps. More precisely, we considered $b i$ oriented planar maps. A bi-orientation of a map is a choice of a direction for each half-edge of the map (thus there are 4 ways of bi-orienting any edge). Seeing maps as a special case of hypermaps, we can describe a bi-orientation as a hyperorientation of the associated hypermap in the way indicated in Figure 13.

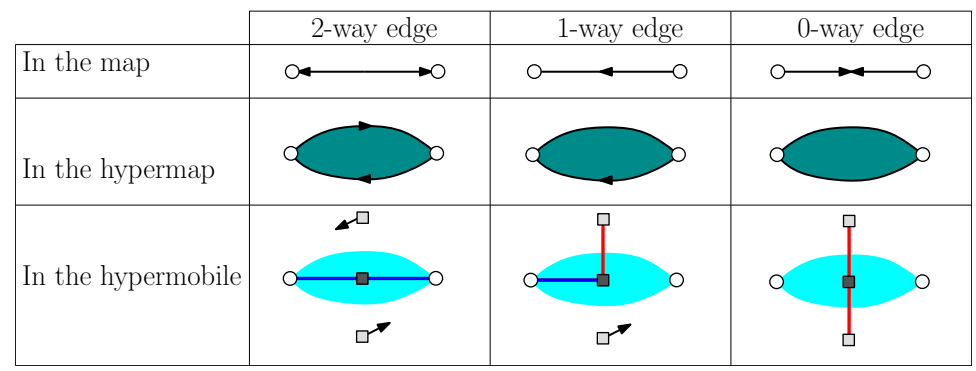

Figure 13. Maps identify to hypermaps by blowing each edge $e$ into a dark face $f$ of degree 2 , the middle-line also shows how to naturally transfer the orientation information so that indegrees are preserved. The bottom line shows that applying the local rules of Figure 7 to (the two edges $\epsilon_{1}, \epsilon_{2}$ of) $f$ is equivalent to applying the local rules given in [4] to the underlying edge $e$.

The master bijection in [4] consists of 3 constructions denoted by $\Phi_{+}, \Phi_{-}, \Phi_{0}$ operating on 3 families $\mathcal{O}_{+}, \mathcal{O}_{-}, \mathcal{O}_{0}$ of bi-orientations. It is easy to check that, under the classical identification of blowing each edge of a map into a dark face of degree 2, the families $\mathcal{O}_{+}$, 

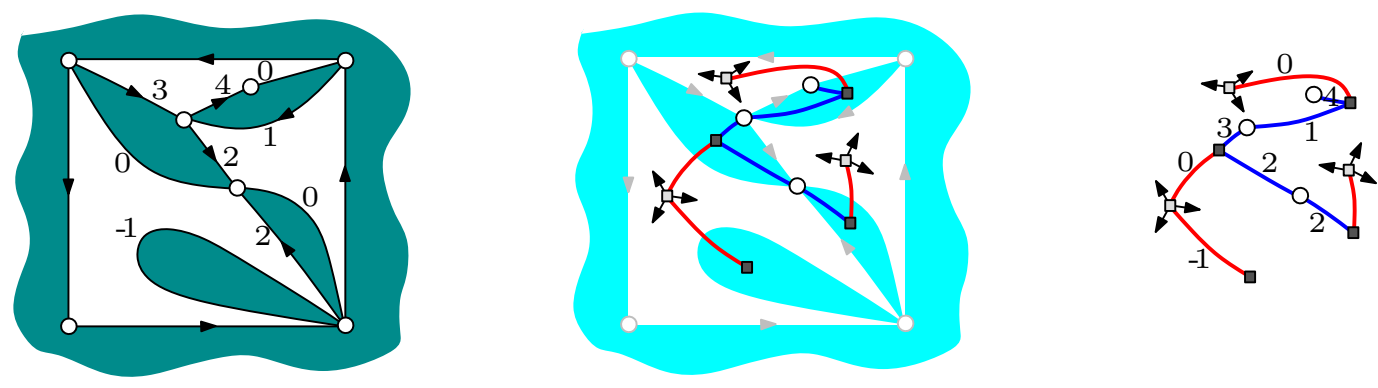

Figure 14. The bijection of Theorem 10 on an example (case $d=4$ ). Left: a dark-rooted hypermap endowed with its unique 4-weighted hyperorientation in $\mathcal{H}_{-}$. Right: the associated 4-weighted hypermobile.

$\mathcal{O}_{-}, \mathcal{O}_{0}$ of bi-orientations considered in [4] identify respectively to the subfamilies of $\mathcal{H}_{+}$, $\mathcal{H}_{-}, \mathcal{H}_{0}$ where all inner dark faces are of degree 2 . Moreover the local rules to carry out the bijections are equivalent under this identification, see Figure 13 . Hence Theorems 4 and 6 extend the results given in [4] about the master bijections for maps.

The proof of Theorems 4 and 6 could actually be obtained using a reduction to the results about the master bijection for maps established in 4 . These in turn, were obtained using results established in [2]. Instead we chose to give a simplified - self-contained - proof in Section 9

\section{BiJeCtions FOR PLANE HYPERMAPS ACCORDING TO THE INGIRTH}

We first define the ingirth of a plane hypermap $H$. A simple cycle $C$ of $H$ is called inward if all the faces incident to $C$ and inside $C$ (on the side of $C$ not containing the outer face) are light. The ingirth of $H$ is then defined as the minimal length of inward cycles. Note that the ingirth of a plane hypermap whose dark faces have degree 2 is equal to the girth of the corresponding map. In this section, we present bijections for dark-rooted hypermaps with control on the face-degrees and on the ingirth.

For $d \geq 1$ and $H$ a dark-rooted hypermap of outer degree $d$, a $d$-weighted hyperorientation of $H$ is a weighted hyperorientation of $H$ such that:

- The 1-way edges have positive weight, the 0 -way edges have non-positive weight.

- Inner vertices have weight $d$.

- Each light face $f$ has weight $d-\operatorname{deg}(f)$.

- Each dark inner face $f$ has weight $d \cdot \operatorname{deg}(f)-d-\operatorname{deg}(f)$.

- Outer vertices and outer edges have weight 1.

Theorem 7. Let d be a positive integer. A dark-rooted hypermap of outer degree $d$ can be endowed with a d-weighted hyperorientation if and only if it has ingirth $d$. In this case, it has a unique $d$-weighted hyperorientation in $\mathcal{H}_{-}$.

The proof of this theorem is postponed to Section 10. We now define the corresponding hypermobiles. For $d \geq 1$, a $d$-weighted hypermobile is a weighted hypermobile such that:

- Edges incident to a round vertex have positive weight, while edges incident to a light square vertex have non-positive weight.

- Round vertices have weight $d$.

- Each light square vertex $v$ has weight $d-\operatorname{deg}(v)$.

- Each dark square vertex $v$ has weight $d \cdot \operatorname{deg}(v)-d-\operatorname{deg}(v)$.

Claim 8. Every d-weighted hypermobile has excess $-d$.

\footnotetext{
${ }^{1}$ In this reduction we would apply the master bijection of 4 to partially oriented maps, and observe that one can characterize the mobiles which are the image of (hyperoriented) hypermaps (because bicolorability of the faces can be detected on the associated mobiles).
} 
Proof. Let $n_{R}, n_{L}, n_{D}$ be respectively the numbers of round vertices, light square vertices, and dark square vertices, let $e_{R}$ (resp. $e_{L}$ ) be the number of edges with a round (resp. light square) extremity, and denote by $e=e_{R}+e_{L}$ the total number of edges (excluding buds), and by $b$ the number of buds (the excess is $e_{R}-b$ ). The total weight at round vertices is $d n_{R}$, the total weight at light square vertices is $d n_{L}-e_{L}-b$, and the total weight at dark square vertices is $d e-d n_{D}-e$. Hence we have $d e-d n_{D}-e=d n_{R}+\left(d n_{L}-e_{L}-b\right)$. Together with $n_{R}+n_{L}+n_{D}=e+1$, this gives $e-e_{L}-b=-d$, hence $e_{R}-b=-d$.

Remark 9. The weights of edges in a $d$-weighted hypermobile are always integers. Indeed, since every vertex has integer weight, no vertex can be incident to exactly 1 edge with a non-integer weight. Hence there cannot exist a non-empty subset of edges with noninteger weights (because any such subset has a vertex of degree 1). Note also that the same argument shows that if the weights of the vertices of a hypermobile are all multiples of a number $k$, then the edge weights are also multiples of $k$.

Given Theorem 7 we can apply the master bijection $\Phi_{-}$for hypermaps. Given the parameter correspondence for $\Phi_{-}$we obtain the following result; see Figure 14 for an example.

Theorem 10. Let $d$ be a positive integer. Dark-rooted hypermaps of outer degree $d$ and ingirth $d$ are in bijection with d-weighted mobiles. Each light (resp. dark) inner face in the hypermap corresponds to a light (resp. dark) square vertex of the same degree in the associated hypermobile.

\section{Counting Plane hypermaps OF IngirTh $d$}

In this section we determine the generating function $F_{d}$ of corner-rooted hypermaps of ingirth $d$ with a dark outer face of degree $d$. Via the master bijection established in Section 3 and Lemma 11 below, this is reduced to counting rooted $d$-weighted hypermobiles (whereas counting dark-rooted hypermaps of ingirth $d$ amounts to counting unrooted $d$-weighted hypermobile which is harder). Then using the classical recursive decomposition of trees at their root we determine $F_{d}$.

Recall that a corner-rooted hypermap is a hypermap with a marked corner. For a cornerrooted hypermap, we define the root-face as the face containing the marked corner, and the ingirth is defined with respect to this face. We now want to use the bijection of Theorem 10 about dark-rooted hypermaps of ingirth $d$ in order to count corner-rooted hypermaps of ingirth $d$. Note that a given face-rooted hypermap with outer degree $d$ can correspond to less than $d$ corner-rooted hypermaps if the face-rooted hypermap has some symmetries. However the master bijection $\Phi_{-}$behaves nicely with respect to symmetries and we get the following lemma.

Lemma 11. Let $H$ be a dark-rooted hyperorientation in $\mathcal{H}_{-}$and let $T=\Phi_{-}(H)$ be the corresponding hypermobile. Let $a$ and $b$ be respectively the number of distinct marked hypermobiles obtained by marking a bud of $T$ and by marking an edge of $T$ having a round extremity. Then the number of distinct corner-rooted maps obtained from $H$ by choosing a root-corner in the root face is $c=a-b$.

Proof. Let $\delta$ be the outer-degree of $H$. By Theorem 10, T has excess $-\delta$, that is, its numbers $\alpha$ and $\beta$ of buds and edges with a round extremity are related by $\alpha-\beta=\delta$. Moreover it is clear from the definition of $\Phi_{-}$that $H$ has a symmetry of order $k$ (which has to be a rotational symmetry preserving the root face) if and only if $T$ has a symmetry of order $k$. In other words, $c=\delta / k$ if and only if $a=\alpha / k$ and $b=\beta / k$. Thus, $c=\delta / k=\alpha / k-\beta / k=$ $a-b$.

Let $d \geq 1$, and let $\mathcal{F}_{d}$ be the family of corner-rooted hypermaps of ingirth $d$ with a dark outer face of degree $d$. Let $F_{d} \equiv F_{d}\left(x_{1}, y_{1} ; x_{2}, y_{2} ; \ldots\right)$ be the generating function of $\mathcal{F}_{d}$ where $x_{k}$ marks the number of light faces of degree $k$, and $y_{k}$ marks the number of dark 
inner faces of degree $k$. Let $A_{d} \equiv A_{d}\left(x_{1}, y_{1} ; x_{2}, y_{2} ; \ldots\right)\left(\right.$ resp. $\left.B_{d} \equiv B_{d}\left(x_{1}, y_{1} ; x_{2}, y_{2} ; \ldots\right)\right)$ be the generating function of $d$-weighted hypermobiles with a marked bud (resp. with a marked edge having a round extremity), where $x_{k}$ marks the number of light square vertices of degree $k$, and $y_{k}$ marks the number of dark square vertices of degree $k$. The bijection of Theorem 10 and Lemma 11 ensure that

$$
F_{d}=A_{d}-B_{d}
$$

We now calculate $A_{d}$ and $B_{d}$, with the help of auxiliary generating functions. A planted $d$-hypermobile is a tree $T$ that can be obtained as one of the two components after cutting a $d$-weighted hypermobile at the middle of an edge $e$. The extremity of $e$ in the chosen component is called the root-vertex of $T$, the half-edge of $e$ in the chosen component is called the root-leg of $T$, and the weight of $e$ is called the root-weight of $T$. For $i \in \mathbb{Z}$, we denote by $\mathcal{W}_{i}$ (resp. $\mathcal{L}_{i}$ ) the family of planted $d$-hypermobiles with root-weight $i$ with a root-vertex which is dark-square (resp. not dark-square). Define $W_{i} \equiv W_{i}\left(x_{1}, y_{1} ; x_{2}, y_{2} ; \ldots\right.$ ) (resp. $\left.L_{i} \equiv L_{i}\left(x_{1}, y_{1} ; x_{2}, y_{2} ; \ldots\right)\right)$ as the generating function of $\mathcal{W}_{i}$ (resp. $\mathcal{L}_{i}$ ) where $x_{k}$ marks the number of light square vertices of degree $k$, and $y_{k}$ marks the number of dark square vertices of degree $k$. Note that $W_{i}=L_{i}=0$ if $i>d$. We also define

$$
W(u)=u+\sum_{k \leq 0} W_{k} u^{k+1}, \quad L(u)=u^{-d+1} \sum_{k \in \mathbb{Z}} L_{k} u^{k} .
$$

We now write equations specifying the series $W_{i}$ and $L_{i}$ using the classical recursive decomposition of trees at the root. As in [4, 5], we will need the following notation: for $k \geq 0$ and $s \geq 0$, define the multivariate polynomial $h_{k}\left(w_{1}, \ldots, w_{s}\right)$ as

$$
h_{k}\left(w_{1}, \ldots, w_{s}\right)=\left[t^{k}\right] \frac{1}{1-\sum_{m=1}^{s} t^{m} w_{m}} .
$$

In other words, $h_{k}$ is the generating function of the compositions of $k$ with weight $w_{i}$ for each part of size $i$.

For $i \geq 1$ any mobile in $\mathcal{L}_{i}$ has a root-vertex $v$ which is round. Hence the children of $v$ are dark square, and the edges incident to $v$ have positive weight. Moreover, the total weight at $v$ is $d$, with a contribution $i$ from the root leg. Hence the total weight of the edges from $v$ to its children is $d-i$. This gives

$$
L_{i}=h_{d-i}\left(W_{1}, \ldots, W_{d-1}\right) \text { for } i \geq 1 .
$$

For $i \leq 0$, a mobile in $\mathcal{L}_{i}$ has a root-vertex $v$ which is light square. Hence the children of $v$ are dark square, and the edges incident to $v$ have non-positive weight. Moreover, the total weight at $v$ is $d-\operatorname{deg}(v)$, with a contribution $i$ from the root-leg. If $v$ has $\operatorname{degree} \delta$, the $\delta-1$ other half-edges incident to $v$ are either buds or are on an edge (with non-positive weight) leading to a dark square vertex. This gives

$$
L_{i}=\sum_{\delta \geq d-i} x_{\delta}\left[u^{d-\delta-i}\right]\left(1+\sum_{k \leq 0} W_{k} u^{k}\right)^{\delta-1} \text { for } i \leq 0 .
$$

In other words,

$$
L_{i}=\left[u^{d-i-1}\right] \sum_{\delta \geq d-i} x_{\delta} W(u)^{\delta-1} \text { for } i \leq 0 .
$$

For $i \in \mathbb{Z}$, a mobile in $\mathcal{W}_{i}$ has a root-vertex $v$ that is dark square. If $v$ has degree $\delta$ then the weight of $v$ is $d \delta-d-\delta$, with a contribution $i$ from the root-leg. Hence

$$
W_{i}=\sum_{\delta \geq 1} y_{\delta}\left[u^{d \delta-d-\delta-i}\right]\left(\sum_{k \in \mathbb{Z}} L_{k} u^{k}\right)^{\delta-1} \text {. }
$$

In other words,

$$
W_{i}=\left[u^{-i-1}\right] \sum_{\delta \geq 1} y_{\delta} L(u)^{\delta-1} \text { for } i \in \mathbb{Z}
$$


Theorem 12. For $d \geq 1$ the generating function $F_{d}(\boldsymbol{x}, \boldsymbol{y})$ of the class $\mathcal{F}_{d}$ of corner-rooted hypermaps of ingirth d having a dark root-face of degree $d$ is given by

$$
F_{d}=L_{0}-\sum_{i=1}^{d} L_{i} W_{i},
$$

where the series $L_{i}$ and $W_{i}$ are specified by (3) and (4) (with $L(u)$ and $W(u)$ defined in (1)).

Moreover

$$
\frac{\partial F_{d}}{\partial x_{k}}=\frac{d}{k}\left[u^{d}\right] W(u)^{k}, \quad \frac{\partial F_{d}}{\partial y_{k}}=\frac{d}{k}\left[u^{-d}\right] L(u)^{k} .
$$

Proof. About the expression of $F_{d}$, we have seen that $F_{d}=A_{d}-B_{d}$. Note that $A_{d}=L_{0}$ (because the marked bud can be turned into a leg of weight 0 ) and $B_{d}=\sum_{i=1}^{d} L_{i} W_{i}$ (because the marked edge $e$ can have any weight $i \in\{1 . . d\}$, and cutting $e$ in its middle yields two planted $d$-hypermobiles that are respectively in $\mathcal{L}_{i}$ and in $\mathcal{W}_{i}$ ). For expressing the partial derivatives of $F_{d}$, we note that $x_{k} \frac{k}{d} \frac{\partial F_{d}}{\partial x_{k}}$ is the generating function of dark-rooted hypermaps with a dark root face of degree $d$, and an additional marked corner in an inner light face of degree $k$. By the bijection $\Phi_{-}$this is also the generating function of $d$-weighted hypermobiles with a marked corner at a light square vertex of degree $k$, which is easily seen to be $x_{k}\left[u^{d}\right] W(u)^{k}$. A similar argument gives the expression for the partial derivative according to $y_{k}$.

Remark 13. The generating function $F_{d}$ of hypermaps can be specialized into a generating function of maps. More precisely, the class $\mathcal{G}_{d}$ of corner-rooted maps of girth $d$ with a root-face of degree $d$, identifies with the set of hypermaps in $\mathcal{F}_{d}$ such that every inner dark face has degree 2. Thus the generating $G_{d}$ of $\mathcal{G}_{d}$ is obtained from $F_{d}$ by setting $y_{2}=1$ and $y_{\delta}=0$ for $\delta \neq 2$. Theorem 12 then gives the expressions of $G_{d}$ given in [5] (upon observing that (4) yields $W_{i}=L_{d-2-i}$, that is, $L_{i}=W_{d-2-i}$.

For any sets $\Delta, \Delta^{\prime}$, the generating function $F_{d, \Delta, \Delta^{\prime}}$ of corner-rooted hypermaps in $\mathcal{F}_{d}$ with inner light face having degree in $\Delta$ and inner dark face having degree in $\Delta^{\prime}$ is obtained by setting $x_{k}=0$ for $k \notin \Delta, y_{k}=0$ for $k \notin \Delta^{\prime}$. We point out that the generating function $F_{d, \Delta, \Delta^{\prime}}$ is algebraic as soon as $\Delta, \Delta^{\prime}$ are both finite (because only a finite number of auxiliary series $W_{i}, L_{i}$ are involved). For instance, for $d=4, \Delta=\{4\}$ and $\Delta^{\prime}=\{3\}$, we have

$$
F_{4,\{4\},\{3\}}=L_{0}-L_{1} W_{1}-L_{2} W_{2}-L_{3} W_{3}-L_{4} W_{4},
$$

where the series $\left\{L_{0}, L_{1}, L_{2}, L_{3}, L_{4}, W_{0}, W_{1}, W_{2}, W_{3}, W_{4}\right\}$ are specified by

$$
\begin{aligned}
& L_{0}=x_{4}\left(1+W_{0}\right)^{3}, L_{1}=W_{1}^{3}+2 W_{1} W_{2}+W_{3}, L_{2}=W_{1}^{2}+W_{2}, L_{3}=W_{1}, L_{4}=1, \\
& W_{0}=2 y_{3} L_{2} L_{3}, W_{1}=y_{3}\left(2 L_{1} L_{3}+L_{2}^{2}\right), W_{2}=2 y_{3} L_{1} L_{2}, W_{3}=y_{3} L_{1}^{2}, W_{4}=2 y_{3} L_{1} .
\end{aligned}
$$

\section{RECOVERING KNOWN BIJECTIONS AS SPECIALIZATIONS}

In this section we show that the bijections described in [7, 8] can be recovered by specializing the bijections of Theorem 10, and the bijections described in [10, 11] can be recovered by specializing the master bijection $\Phi_{0}$ (in a way which can be thought of as the case $d=0$ of Theorem 10.

5.1. The Bousquet-Mélou Schaeffer bijection for bipartite maps. Recall that a bipartite map is a map whose vertices can be colored in black and white such that each edge connects a black vertex to a white vertex. A 1-leg bipartite map is a bipartite map with a marked vertex of degree 1 ; this vertex is considered as black, hence it fixes the coloring of all vertices. Note that 1-leg bipartite maps are dual to dark-rooted hypermaps of outer degree 1. In [8], Bousquet-Mélou and Schaeffer have given a bijection between 1-leg bipartite maps and so-called well-charged blossom trees. We show here that the bijection in [8] is equivalent (up to duality) to the case $d=1$ of the bijection of Theorem 10 . 


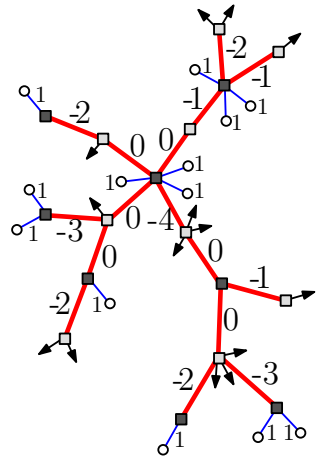

(a)

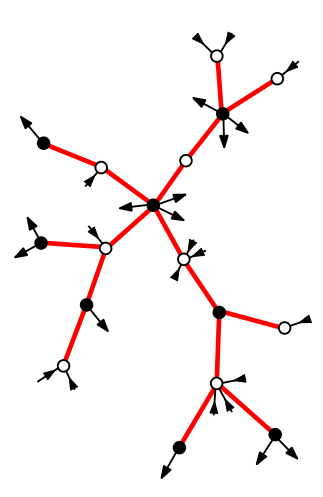

(b)

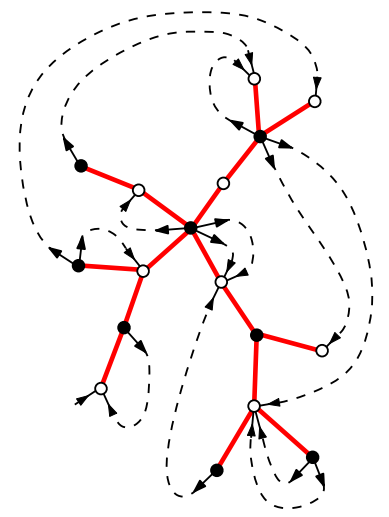

(c)

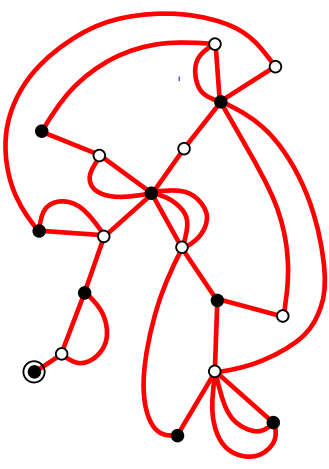

(d)

Figure 15. (a) A 1-weighted hypermobile T. (b) The corresponding wellcharged blossom tree $T^{\prime}$ (inbuds and outbuds are represented by ingoing and outgoing arrows). (c)-(d) The closure of $T^{\prime}$, which is the same as the closure of $T$.

A blossom tree is a bipartite plane tree (with black and white vertices) with dangling half-edges. The dangling half edges at black and white vertices are called outbuds and inbuds respectively (the terminology in [8] is actually buds and leaves but this is confusing in the present context). A planted subtree of a blossom tree $T$ is a subtree that can be obtained as one of the two components after cutting at the middle of an edge $e$ of $T$ (not at a bud). The extremity of $e$ in the chosen component is called the root-vertex of the planted subtree. The charge of a blossom tree or subtree is its number of inbuds minus its number of outbuds $2^{2}$ A blossom tree is well-charged if it has charge 1 and every planted subtree has charge at most 1 when its root-vertex is black, and at least 0 when its root-vertex is white. A well-charged blossom tree is represented in Figure 15(b).

We first show that well-charged blossom trees identify to 1-weighted hypermobiles, see Figure 15(a)-(b). By definition the round vertices of 1-weighted hypermobiles have weight 1 hence are leaves (i.e., vertices of degree 1). Thus, forgetting the weights, a 1-weighted hypermobile identifies to a blossom tree by interpreting dark and light square vertices as black and white vertices, round vertices as outbuds, and buds as inbuds. Hence we define the charge of a 1-weighted hypermobile or of a planted 1-hypermobile as its number of buds minus its number of round vertices. An easy induction (using the same recursive decomposition as in Section 4 ensures that a planted 1-hypermobile of root-weight $w$ such that the root-vertex is dark square (resp. light square) has charge $-w$ (resp. $w+1$ ). Thus the fact that the edge weights of 1-hypermobiles are positive for edges having a round endpoint and non-positive otherwise corresponds to the fact that the associated blossom tree is well-charged. Thus well-charged blossom trees identify to 1-weighted hypermobiles (if one starts from a well-charged blossom tree, the weights on the corresponding 1-hypermobile are determined: each edge $e$ gets a weight $c-1$, where $c$ is the charge of the planted subtree rooted on the dark square endpoint of $e$ ).

The bijection in [8] associates a 1-leg bipartite map to each well-charged blossom tree using a closure operation; see Figure 15(b)-(d). More precisely, for a well-charged blossom tree $T$ one considers the cyclic word $w_{T}$ obtained by walking clockwise around $T$ and encoding outbuds and inbuds by letters $a$ and $\bar{a}$ respectively. Then, the cw-matching outbuds and inbuds of $T$ are joined into edges. Since the charge of $T$ is 1 , there remains 1 unmatched inbud. The result of the closure operation is therefore a 1-leg bipartite map,

\footnotetext{
${ }^{2}$ This notion of charge is taken from 8 and is not related to the notion of charge (which constraints the cycle-lengths) to be introduced in Section 6
} 


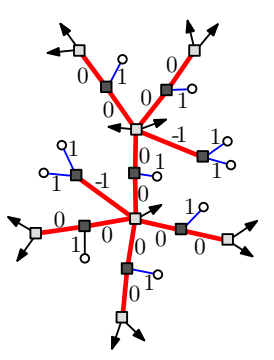

(a)

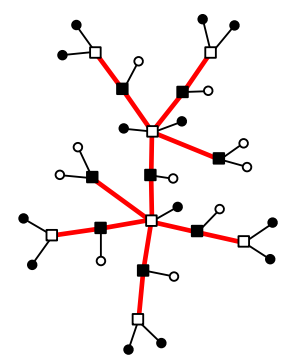

(b)

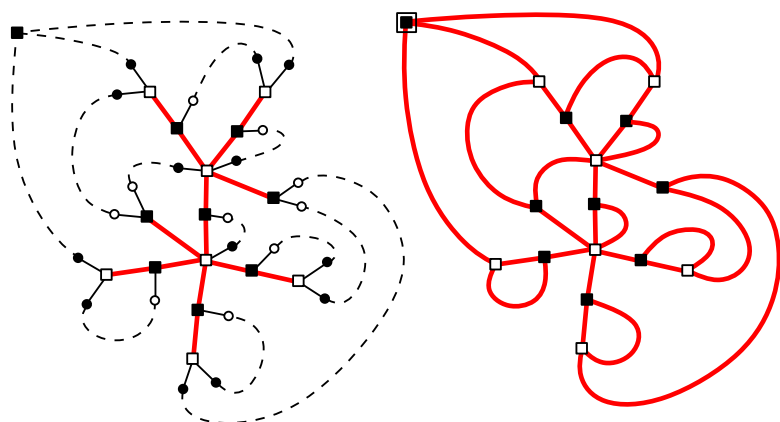

(c)

(d)

FIgURE 16. (a) A $p$-weighted hypermobile $T$ associated with a dark-rooted $p$-constellation, after all weights have been divided by $p=3$. (b) The corresponding $p$-Eulerian tree $T^{\prime}$. (c)-(d) The closure of $T^{\prime}$, which is the same as the closure of $T$.

if one interprets the unmatched inbud as the leg leading to a black vertex of degree 1 . Moreover, it is clear that this closure operation of [8] applied to a well-charged blossom tree is equivalent to the closure operation of $\Psi_{+}$(as formulated in Section 2.4) applied to the corresponding 1-weighted hypermobile. To summarize we obtain:

Proposition 14. The blossom trees of [8] identify to 1-weighted hypermobiles. Under this identification the bijection of $[8$ is the same as the case $d=1$ of the bijection of Theorem 10

5.2. The Bousquet-Mélou Schaeffer bijection for constellations. For any fixed $p \geq$ 2 , we call $p$-constellation a (planar) hypermap where the degree of each dark face is $p$ and the degree of each light face is a multiple of $p$ (these maps encode certain factorizations in the symmetric group; see 25). In 7], Bousquet-Mélou and Schaeffer have given a bijection between dark-rooted $p$-constellations and so-called $p$-Eulerian trees. We show here that the bijection in [7] is equivalent to the case $d=p$ of the bijection of Theorem 10 applied to $p$-constellations. Before discussing the equivalence, we show that $p$-constellations have ingirth $p$.

Lemma 15. A p-constellation has ingirth $p$.

Proof. Let $K$ be a $p$-constellation, and let $C$ be an inward cycle of $K$. Clearly the length of $C$ equals $A-B$, where $A$ is the total degree of all light faces inside $C$ and $B$ is the total degree of all dark faces inside $C$. Since all faces (dark or light) have degree a multiple of $p$, the length of $C$ is a multiple of $p$, hence is at least $p$.

We now explicit the equivalence of the bijection in [7] with the the case $d=p$ of Theorem 10 applied to $p$-constellations. A $p$-Eulerian tree is a bipartite plane tree (with black and white vertices) satisfying:

- Each black inner node (non-leaf vertex) has degree $p$ and has either $n=1$ or $n=2$ neighbors that are inner nodes. This black vertex is said to be of type $n \in\{1,2\}$.

- Each white inner node has degree of the form $p i$ with $i \geq 1$, and it has $i-1$ neighbors that are black inner nodes of type 1.

We first show that $p$-Eulerian trees identify with the $p$-weighted hypermobiles corresponding to $p$-constellations. A $p$-weighted hypermobile $T$ corresponds to a $p$-constellation if all dark square vertices have degree $p$ and all light square vertices have degree multiple of $p$. In this case, by Remark 9, all the edge weights of $T$ are multiple of $p$, and we denote by $T^{\prime}$ the weighted-hypermobile obtained by dividing every weight by $p$. In $T^{\prime}$ the weight of each round vertex is 1 , the weight of each dark square vertex is $p-2$, and the weight of each light square vertex of degree $p i$ is $1-i$. Since round vertices have weight 1 they are leaves. Since a dark square vertex has degree $p$ and weight $p-2$, it has either $p-1$ round neighbors 
and $n=1$ light square neighbor (and the edge to the light square neighbor has weight -1 ) or it has $p-2$ round neighbors and $n=2$ light square neighbors (and the edges to the light square neighbors have weight 0 ). This dark square vertex is said to be of type $n \in\{1,2\}$. Since a light square vertex of degree $p i$ has weight $1-i$, it has $i-1$ dark square neighbors of type 1 . Thus $p$-weighted hypermobiles corresponding to $p$-constellations identify with $p$-Eulerian trees if one interprets buds as black leaves, round vertices as white leaves, dark square vertices as black inner nodes, and light square vertices as white inner nodes. Indeed, if one starts from a $p$-Eulerian tree, the corresponding hypermobile is obtained by giving a weight $w$ to each edge $e=(u, v)$ connecting a black inner node $u$ to a white inner node $v$, where $w=-1$ if $u$ has type 1 and $w=0$ if $u$ has type 2 .

The bijection in [7] associates a dark-rooted $p$-constellation with such a tree $T$ using a closure operation (see Figure 16(b)-(d)). More precisely, a counterclockwise walk around the outer face of $T$ sees a succession of black leaves and white leaves, and we consider the cw-matching when black leaves are interpreted as letters $a$, and white leaves as letters $\bar{a}$. The pairs of cw-matching leaves are joined by edges. It can be shown that a $p$-Eulerian tree has an excess of $p$ black leaves over white leaves. Hence after the cw-matching, there remain $p$ unmatched black leaves (all in the outer face) and these are merged into a black vertex of degree $p$ taken as the root-vertex. This yields a vertex-rooted bipartite map where black vertices have degree $p$ and white vertices have degree multiple of $p$. Hence the dual of the obtained bipartite map is a dark-rooted $p$-constellation.

It is clear that the closure mapping (as formulated in Section 2.4) applied to a $p$-weighted hypermobile of a $p$-constellation is equivalent to the closing mapping of [7] applied to the corresponding $p$-Eulerian tree. To summarize we obtain:

Proposition 16. For $p \geq 2$, the $p$-Eulerian trees of [7] identify to p-weighted hypermobiles that are associated with dark-rooted p-constellations. Under this identification the bijection of [7] is the same as the case $d=p$ of the bijection of Theorem 10 applied to p-constellations.

Remark 17. Since the two bijections are the same, the inverse mappings from constellations to decorated trees also coincide. In both cases, the decorated tree is recovered as the complemented dual of a forest: in our case the forest $F$ is made of the directed edges of the canonical $p$-weighted orientation, while in [7] the forest $F^{\prime}$ is the so-called rank-forest (see 7], in particular Section 5.2 and Proposition 6.2). Our rules to obtain the $p$-weighted hypermobile from $F$ can be checked to coincide with the rules given in [7] to obtain the $p$-eulerian tree from $F^{\prime}$. So $F$ is the same as $F^{\prime}$.

5.3. The Bouttier Di Francesco Guitter bijections for Eulerian maps. In [10], Bouttier, Di Francesco and Guitter have given a bijection for vertex-rooted hypermaps. In [11] this bijection was generalized to vertex-rooted hypermaps with some "blocked edges". We show here that these bijections can be obtained as specializations of the master bijection $\Phi_{0}$ (and can be thought of as "the case $d=0$ " of Theorem 10 .

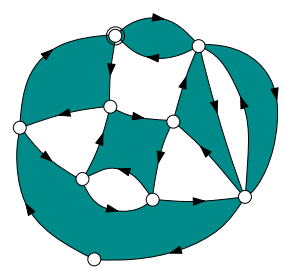

(a)

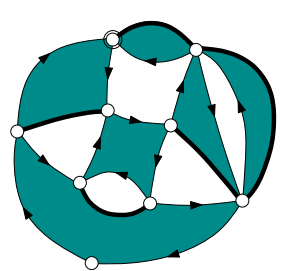

(b)

FIgURE 17. (a) A vertex-rooted hypermap endowed with its dark-light orientation. (b) The same hypermap with some blocked edges, endowed with its dark-light hyperorientation (blocked edges are 0-way, other edges are 1-way). 


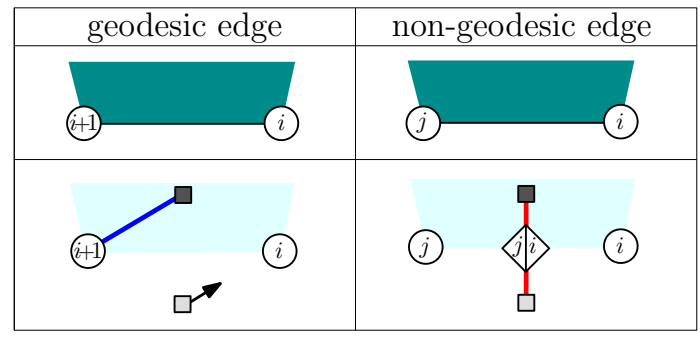

FIGURE 18. Local rules applied to the edges of a vertex-rooted hypermap, according to the distance-labelling.
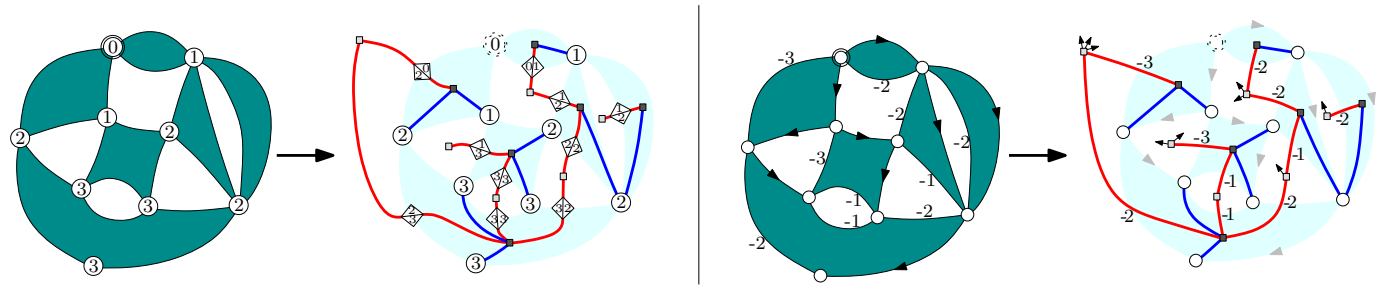

FiguRE 19. Left: the bijection of 10 between vertex-rooted hypermaps and well-labeled hypermobiles. Right: the bijection seen as a specialization of the master bijection $\Phi_{0}$.

Let $M$ be a vertex-rooted hypermap, and let $v_{0}$ be its root-vertex. The hyperorientation $\Omega$ of $M$ such that each edge has a dark face on its right is called the dark-light hyperorientation of $M$; see Figure 17(a). We give to each vertex $v$ of $M$ the label $\ell(v)$ equal to the length of a shortest directed path of $\Omega$ from $v_{0}$ to $v$. For each edge $e=(u, v)$ (oriented from $u$ to $v$ in $\Omega$ ), the labels of $u$ and $v$ clearly satisfy $\ell(v) \leq \ell(u)+1$. We call $e$ geodesic if $\ell(v)=\ell(u)+1$ and non-geodesic otherwise. One associates with $M$ a hypermobile $T$ without buds, but with labels, by applying to each edge the rule indicated in Figure 18 More precisely, $T$ has labels on the round vertices, called vertex labels, and on each side of any edge incident to a light square vertex, called edge labels. Moreover, it is easy to see that $T$ satisfies the following properties:

- Vertex labels are positive and edge labels are non-negative.

- In clockwise order around a dark square vertex, any two consecutive labels $\ell, \ell^{\prime}$ satisfy $\ell^{\prime} \leq \ell$ if $\ell, \ell^{\prime}$ are edge-labels on the same edge, $\ell^{\prime}=\ell+1$ if $\ell^{\prime}$ is a vertexlabel, and $\ell^{\prime}=\ell$ in the other cases.

- In clockwise order around a light square vertex, any two consecutive edge-labels $\ell, \ell^{\prime}$ satisfy $\ell^{\prime} \geq \ell$ if $\ell, \ell^{\prime}$ are on the same edge, and $\ell^{\prime} \leq \ell$ otherwise.

We call well-labeled mobile a labeled hypermobile satisfying these conditions; see Figure 19 for an example.

Bouttier, Di Francesco and Guitter have shown in [10] that applying the local rules of Figure 18 gives a bijection between vertex-rooted hypermaps and well-labeled mobiles. Now we explain how to reformulate the distance-labelling and the well-labeled mobiles, and the connection with the master bijection $\Phi_{0}$.

First, we show that the distance-labelling can be encoded as a weighted hyperorientation; see Figure 19 right part. We call geodesic hyperorientation of $M$ the weighted hyperorientation such that each geodesic edge is 1 -way with weight 0 , and each non-geodesic edge $e=(u, v)$ (with the dark face on its right) is 0 -way with weight $\ell(v)-\ell(u)-1$. The geodesic hyperorientation satisfies the following conditions:

- The weight of an edge $e$ is 0 if $e$ is 1 -way, and is negative if $e$ is 0 -way.

- The weight of a vertex is 0 , and the weight of a face $f$ (light or dark) is $-\operatorname{deg}(f)$. 
We call 0-weighted a hyperorientation satisfying these conditions. Note that the geodesic hyperorientation has two additional properties: it is accessible from $v_{0}$ and it is acyclic; hence it is in $\mathcal{H}_{0}$.

Lemma 18. A vertex-rooted hypermap $M$ has a unique 0 -weighted hyperorientation in $\mathcal{H}_{0}$; it is its geodesic hyperorientation.

Proof. Let $M$ be a vertex-rooted hypermap, and let $v_{0}$ be its the root-vertex. We call admissible labelling of $M$ a labelling $L$ of its vertices (each vertex $v$ has a label $L(v) \in$ $\mathbb{Z}$ ) such that $L\left(v_{0}\right)=0$ and for each edge $e=(u, v)$ (with the dark face on its right) $L(v) \leq L(u)+1$. One can associate to such a labelling a 0 -weighted hyperorientation exactly in the same way as we have done for the distance labelling. And this actually gives a bijection between admissible labellings and 0-weighted hyperorientations of $M$. We have already seen that the 0 -weighted hyperorientation associated with the distance-labelling is in $\mathcal{H}_{0}$. Note that any admissible labelling $L$ satisfies $L(v) \leq \ell(v)$ for all vertices (because the labels increase by at most 1 along each edge of a geodesic path ending at $v$ ). If $L$ is not equal to $\ell$, consider a vertex $v$ such that $L(v)<\ell(v)$ (note that $v \neq v_{0}$ ) and $L(v)$ is the smallest possible. Assume there is a neighbor $v^{\prime}$ of $v$ such that $L\left(v^{\prime}\right)<L(v)$, that is, $L\left(v^{\prime}\right)=L(v)-1$. Since $\ell\left(v^{\prime}\right) \geq \ell(v)-1$ we reach the contradiction that $L\left(v^{\prime}\right)<\ell\left(v^{\prime}\right)$. Hence $v$ is not accessible from $v_{0}$ in the 0 -weighted hyperorientation associated with $L$, so the hyperorientation is not in $\mathcal{H}_{0}$.

Second, we show that the well-labeled mobiles can be encoded as weighted (unlabeled) hypermobiles; see Figure 19 bottom part. From a well-labeled mobile $T$ we construct a weighted hypermobile $\theta(T)$ as follows. Give weight 0 to each edge incident to a round vertex, and give weight $\ell-r-1$ to each edge $e$ incident to a light square vertex $u$, where $\ell$ and $r$ are the edge labels on the left side and right side of $e$ looking from $u$. In each corner $c$ of $T$ at a light square vertex $u$ between two consecutive edges $e, e^{\prime}$ (in clockwise order), insert $r-\ell$ buds in the corner $c$ where $r$ is the edge label on the right side of $e$ (looking from $u$ ), and $\ell$ is the edge label on the left side of $e^{\prime}$. Then delete all the labels. The obtained hypermobile $\theta(T)$ satisfies the following conditions:

- Edges incident to a round vertex have weight 0 (hence round vertices have weight 0 ), while edges incident to a light square vertex have negative weight.

- Each square vertex $v$ (light or dark) has weight $-\operatorname{deg}(v)$.

We call 0-weighted a hypermobile satisfying these conditions. Clearly $\theta$ is a bijection between well-labeled mobiles and 0-weighted hypermobiles. We can now show that the bijection of [1] can be obtained as a specialization of $\Phi_{0}$; see Figure 19

Proposition 19. The master bijection $\Phi_{0}$ yields a bijection between vertex-rooted hypermaps and 0-weighted hypermobiles. This bijection coincides with the Bouttier Di Francesco Guitter bijection, up to the identification of well-labeled mobiles with 0-weighted hypermobiles.

Remark 20. The bijection [1], as reformulated in Proposition 19, can be thought of as the "case $d=0$ " of Theorem 10. Indeed, one can think of a vertex-rooted hypermap as a dark-rooted hypermap of degree 0 . Then the definition of 0 -weighted hyperorientation coincides with the case $d=0$ of $d$-weighted hyperorientations given in Section 3 , except that the weight 0 are authorized on 1 -way edges instead of on 0 -way edge. Also the definition of 0 -weighted hypermobile coincides with the case $d=0$ of $d$-weighted hypermobile given in Section 3 , except that the weight 0 is authorized on edges incident to round vertices instead of on edges incident to light square vertices.

Proof. It is easy to prove that 0-weighted hypermobiles have excess 0 . Hence the master bijection $\Phi_{0}$ clearly yields a bijection between 0 -weighted hypermobiles and 0 -weighted vertex-rooted hyperorientations in $\mathcal{H}_{0}$. By Lemma 18 , the latter family identifies to the family of vertex-rooted hypermaps. 

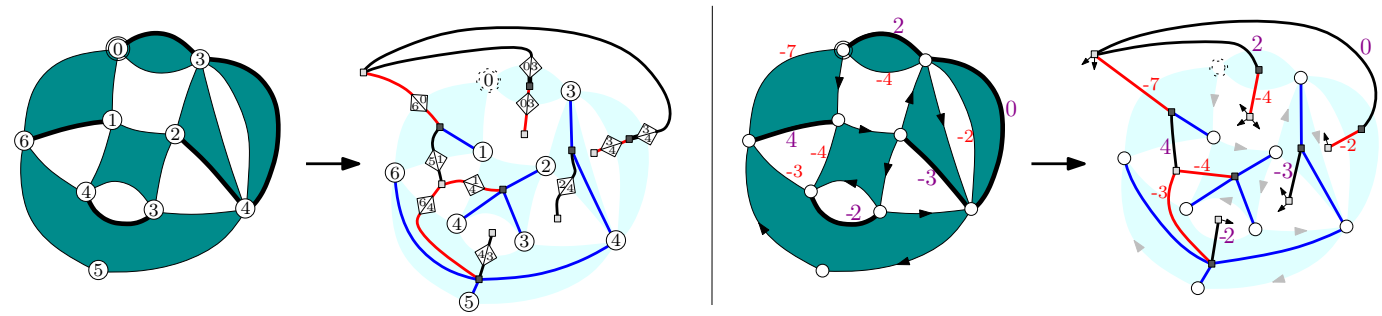

FIGURE 20. Left: The bijection of [1] between vertex-rooted hypermaps with blocked edges and generalized well-labeled mobiles. Right: the same bijection seen as a specialization of $\Phi_{0}$.

Now one easily verifies from Figure 18 that, if $M$ is a vertex-rooted hypermap and $T$ is the associated well-labeled mobile, then $\theta(T)$ is obtained from the geodesic hyperorientation by applying the local rules of Figure 7 that is, by applying $\Phi_{0}$.

We now discuss the bijection given in [11, which is an extension of the bijection in [10 for vertex-rooted hypermaps with blocked edges. Let $M$ be a vertex-rooted hypermap, with $v_{0}$ the root-vertex, and let $X$ be a subset of the edges of $M$ called blocked edges. Let $\Omega_{X}$ be the hyperorientation of $M$ where the edges in $X$ are 0 -way and the edges not in $X$ are 1-way. The subset $X$ is called admissible if $\Omega_{X}$ is accessible from $v_{0}$. A pair $(M, X)$, with $M$ a vertex-rooted hypermap and $X$ an admissible subset of edges of $M$, is shortly called a (vertex-rooted) hypermap with blocked edges.

The bijection of [11] proceeds similarly as the one above (which corresponds to the case $X=\emptyset)$. Namely, we give to each vertex $v$ a label equal to the minimal length of the directed paths in $\Omega_{X}$ from $v_{0}$ to $v$. We call $e=(u, v)$ (with the dark face on the right of e) geodesic if it is not blocked and $\ell(v)=\ell(u)+1$, and non-geodesic otherwise. Then a labeled mobile is associated with $(M, X)$ by applying the rule of Figure 18, and marking as blocked the edges of the mobile corresponding to blocked edges of $M$. The associated labeled mobiles, called generalized well-labelled mobiles satisfy the same conditions as welllabelled mobiles, with the only difference that there can be some blocked edges incident to light square vertices and that the difference between the edge-labels on the two sides of a blocked edge is arbitrary.

As above we can encode the distance-labelling by a weighted hyperorientation. More precisely, we define the geodesic hyperorientation as follows: each geodesic edge is oriented 1-way and given weight 0 , each non-geodesic edge $e=(u, v)$ (with the dark face on the right of $e$ ) is oriented 0 -way and given weight $\ell(v)-\ell(u)-1$. The geodesic hyperorientation satisfies:

- The weight of an edge $e$ is 0 if $e$ is directed, and is negative if $e$ is non-blocked and 0 -way.

- The weight of a vertex is 0 , and the weight of a face $f$ (light or dark) is $-\operatorname{deg}(f)$. A hyperorientation satisfying these conditions is called a generalized 0-weighted hyperorientation. The geodesic hyperorientation has two additional properties: it is accessible from $v_{0}$ and it is acyclic; hence it is in $\mathcal{H}_{0}$.

Lemma 21. A vertex-rooted hypermap with blocked edges $(M, X)$ has a unique generalized 0 -weighted hyperorientation in $\mathcal{H}_{0}$; it is the geodesic hyperorientation.

Proof. The proof is similar to the proof of Lemma 18 Let $(M, X)$ be a vertex-rooted hypermap with blocked edges, and let $v_{0}$ be its root-vertex. We call admissible labelling of $M$ a labelling $L$ of its vertices such that $L\left(v_{0}\right)=0$ and for each non-blocked edge $e=(u, v)$ (with the dark face on the right of $e$ ) $L(v) \leq L(u)+1$. As before there is a bijection between the admissible labellings and the generalized 0 -weighted hyperorientations of $M$. 
Moreover any admissible labelling $L$ which is not the distance-labelling $\ell$ is associated with a hyperorientation which is not accessible, hence not in $\mathcal{H}_{0}$.

We call generalized 0-weighted hypermobile a hypermobile with some marked edges incident to light-square vertices, such that the following conditions hold:

- Edges incident to a round vertex have weight 0 (hence each round vertex has weight 0 ), and non-marked edges incident to a light square have negative weight.

- Each square vertex $v$ (light or dark) has weight $-\operatorname{deg}(v)$.

Similarly as in the case without blocked edges, generalized well-labeled mobiles can be identified to generalized 0-weighted hypermobiles. We now state how the bijection of [11] can be obtained as a specialization of $\Phi_{0}$; see Figure 20 .

Proposition 22. The master bijection $\Phi_{0}$ yields a bijection between vertex-rooted hypermaps with blocked edges and generalized 0-weighted hypermobiles. This bijection coincides with the bijection of [11, up to the identification of generalized well-labeled mobiles with generalized 0-weighted hypermobiles.

Proof. The proof is very similar to the one of Proposition 19 and is left to the reader.

\section{BIJECTIONS FOR HYPERMAPS WITH GENERAL CYCLE-LENGTH CONSTRAINTS}

In this section we consider a far-reaching generalization of the girth constraints considered in Section 3, and obtain bijections for hypermaps satisfying these constraints.

We call charge function $\sigma$ of a hypermap $H$ the assignment of a real number $\sigma(a)$, called charge, to each vertex and face $a$ of $H$. The pair $(H, \sigma)$ is called a charged hypermap. We call total charge, and denote it by $\sigma_{\text {total }}$, the sum of all the charges of the hypermap. We will now define some cycle-length constraints on charged hypermaps. A light region of $H$ is a proper subset $R$ of the faces of $H$ such that any face in $R$ sharing an edge with a face not in $R$ is light. We say that an edge or a vertex is strictly inside a light region $R$ if all its incident faces are in $R$. We denote

$$
\sigma(R)=\sum_{f \text { face inside } R} \sigma(f)+\sum_{v \text { vertex strictly inside } R} \sigma(v) .
$$

The boundary of a light region $R$ is the set of edges incident both to a face in $R$ and to a face not in $R$. We denote by $\partial R$ the boundary of $R$ and by $|\partial R|$ its cardinality.

Definition 23. Let $H$ be a hypermap, and let $\sigma$ be a charge function. If the hypermap $H$ is dark-rooted (resp. light-rooted, vertex-rooted), we say that $H$ satisfies the $\sigma$-girth condition if every light region $R$ satisfies $|\partial R| \geq \sigma(R)$ with strict inequality if all the outer vertices are strictly inside $R$ (resp. if one of the outer edges is strictly inside $R$, if the root-vertex is strictly inside $R$ ).

Various girth constraints can be realized as a $\sigma$-girth condition by choosing an appropriate charge function $\sigma$; examples are given in Section 7 .

We will now characterize the $\sigma$-girth condition by the existence of certain hyperorientations.

Definition 24. Let $(H, \sigma)$ be a charged hypermap. If $H$ is light-rooted or vertex-rooted, we call $\sigma$-weighted hyperorientation of $H$ a weighted hyperorientation such that:

(i) the weight of 1-way edges is positive, and the weight of 0-way edges is non-positive,

(ii) the weight of every light face $f$ is $\sigma(f)-\operatorname{deg}(f)$,

(iii) the weight of every inner dark face $f$ is $-\sigma(f)-\operatorname{deg}(f)$,

(iv) the weight of every vertex $v$ is $\sigma(v)$.

If $H$ is dark-rooted, we call $\sigma$-weighted hyperorientation of $H$, a weighted hyperorientation satisfying (i), (ii), (iii) and 
(iv') the weight of every inner vertex $v$ is $\sigma(v)$, the weight of every outer vertex $v$ is $\sigma(v)+1$, the weight of every outer edge is 1, and the weight of the dark outer face $f_{0}$ is $-\sigma\left(f_{0}\right)$.

We now state the key result for dark-rooted hypermaps. We say that a charge function $\sigma$ fits a dark-rooted hypermap $H$ if $H$ satisfies the $\sigma$-girth condition, the charge of every inner vertex is positive, the charge of every outer vertex is 0 , the charge of the dark outer face $f_{0}$ is $-\operatorname{deg}\left(f_{0}\right)$, and $\sigma_{\text {total }}=0$.

Theorem 25. Let $H$ be a dark-rooted hypermap, and let $\sigma$ be a charge function. The hypermap $H$ admits a $\sigma$-weighted hyperorientation in $\mathcal{H}_{-}$if and only if $\sigma$ fits $H$ and the outer face of $H$ is simple. Moreover, in this case $H$ admits a unique $\sigma$-weighted hyperorientation in $\mathcal{H}_{-}$.

It will be shown in Section 7 that Theorem 7 is a special case of Theorem 25 corresponding to a particular choice of charge function. We now state the analogous result for light-rooted and vertex-rooted hypermaps. We say that a charge function $\sigma$ fits a light-rooted hypermap $H$ if $H$ satisfies the $\sigma$-girth condition, the charge of every vertex is positive, the charge of the light outer face $f_{0}$ is $\operatorname{deg}\left(f_{0}\right)$, and $\sigma_{\text {total }}=0$.

Theorem 26. Let $H$ be a light-rooted hypermap, and let $\sigma$ be a charge function. The hypermap $H$ admits a $\sigma$-weighted hyperorientation in $\mathcal{H}_{+}$if and only if $\sigma$ fits $H$. Moreover, in this case $H$ admits a unique $\sigma$-weighted hyperorientation in $\mathcal{H}_{+}$.

We say that a charge function $\sigma$ fits a vertex-rooted hypermap if $H$ satisfies the $\sigma$-girth condition, the charge of every non-root vertex is positive, the charge of the root-vertex is 0 , and $\sigma_{\text {total }}=0$.

Theorem 27. Let $H$ be a vertex-rooted hypermap, and let $\sigma$ be a charge function. The hypermap $H$ admits a $\sigma$-weighted hyperorientation in $\mathcal{H}_{0}$ if and only if $\sigma$ fits $H$. Moreover, in this case $H$ admits a unique $\sigma$-weighted hyperorientation in $\mathcal{H}_{0}$.

The proof of Theorems 25, 26 and 27 are postponed to Section 10

We will now obtain bijections for charged hypermaps using the master bijections $\Phi_{-}$, $\Phi_{+}$and $\Phi_{0}$. We call fittingly charged hypermap a charged hypermap such that $\sigma$ fits $H$. We call consistently-weighted a hypermobile with weights in $\mathbb{R}$ such that the weights of edges incident to round vertices are positive, while the weights of edges incident to light square vertices are non-positive. We will now show that fittingly charged hypermaps are in bijection with consistently-weighted hypermobiles.

We call charge of a vertex $u$ of a hypermobile the quantity

- $w(u)$ if $u$ is a round vertex,

- $w(u)+\operatorname{deg}(u)$ if $u$ is a light square vertex,

- $-w(u)-\operatorname{deg}(u)$ if $u$ is a dark square vertex.

We now relate the excess of a hypermobile to the charges.

Lemma 28. The excess of a hypermobile of vertex-set $V$ is $-\sum_{v \in V} \sigma(v)$, where $\sigma(v)$ denotes the charge of vertex $v$.

Proof. Let $T$ be a hypermobile. Let $R, D$, and $L$ be respectively the sets of round vertices, dark square vertices, and light square vertices of $T$. Let $b, e_{R}$, and $e_{L}$ be respectively the number of buds, edges incident to a round vertex, and edges incident to a light square vertex. By definition, the excess of $T$ is $e_{R}-b$. If we denote by $w(u)$ and $\sigma(u)$ the weight 
and charge of a vertex $u$, we get

$$
\begin{aligned}
\sum_{u \in R} w(u) & =\sum_{u \in R} \sigma(u), \\
\sum_{u \in L} w(u) & =-e_{L}-b+\sum_{u \in L} \sigma(u), \\
\sum_{u \in D} w(u) & =-e_{R}-e_{L}-\sum_{u \in D} \sigma(u) .
\end{aligned}
$$

Plugging these relations in $\sum_{u \in R} w(u)+\sum_{u \in L} w(u)=\sum_{u \in D} w(u)$ gives $e_{R}-b=-\sum_{v \in R \cup L \cup D} \sigma(v)$, as wanted.

A hyperorientation is called consistently-weighted if the weight of every 1-way edge is a positive real number, and the weight of every 0 -way edge is a non-positive real number. By Theorem 25, the set of fittingly charged dark-rooted hypermaps such that the outer face is simple identifies with the set of consistently-weighted hyperorientations in $\mathcal{H}_{-}$such that the weight of every outer edge is 1 . Moreover,

- the charge of an inner vertex $v$ of $H$ is $\sigma(v)=w(v)$,

- the charge of an inner light face $f$ of $H$ is $\sigma(f)=w(f)+\operatorname{deg}(f)$,

- the charge of an inner dark face $f$ of $H$ is $\sigma(f)=-w(f)-\operatorname{deg}(f)$.

Hence by applying the master bijection $\Phi_{-}$and keeping track of the parameter-correspondences we obtain:

Theorem 29. The mapping $\Phi_{-}$gives a bijection between the set of fittingly charged darkrooted hypermaps such that the outer face is simple, and the set of consistently-weighted hypermobiles with negative excess. Moreover, each light (resp. dark) inner face of degree $\delta$ and charge $x$ of the hypermap corresponds to a light (resp. dark) square vertex of degree $\delta$ and charge $x$ of the associated charged hypermobile. Also each inner vertex of charge $x$ in the hypermap corresponds to a round vertex of charge $x$ of the associated charged hypermobile. Lastly, the outer degree of the hypermap corresponds to minus the excess of the associated hypermobile.

We will see below (see Lemma 37) that Theorem 10 corresponds to a special case of Theorem 29. We now consider light-rooted hypermaps. Similarly as above, using Theorem 26 and applying the master master bijection $\Phi_{+}$we obtain:

Theorem 30. The mapping $\Phi_{+}$gives a bijection between the set of fittingly charged lightrooted hypermaps and the set of consistently-weighted hypermobiles with positive excess. Moreover, each light (resp. dark) inner face of degree $\delta$ and charge $x$ of the hypermap corresponds to a light (resp. dark) square vertex of degree $\delta$ and charge $x$ of the associated hypermobile. Also each vertex of charge $x$ in the hypermap corresponds to a round vertex of charge $x$ of the associated hypermobile. Lastly, the outer degree of the hypermap corresponds to the excess of the associated hypermobile.

Similarly, using Theorem 27 and applying the master master bijection $\Phi_{0}$ we obtain:

Theorem 31. The mapping $\Phi_{0}$ gives a bijection between the set of fittingly charged vertexrooted hypermaps and the set of consistently-weighted hypermobiles with excess zero. Moreover, each light (resp. dark) face of degree $\delta$ and charge $x$ of the hypermap corresponds to a light (resp. dark) square vertex of degree $\delta$ and charge $x$ of the associated hypermobile. Also each non-root vertex of charge $x$ in the hypermap corresponds to a round vertex of charge $x$ of the associated hypermobile.

We will use Theorem 29 and 30 in the next section to count annular hypermaps. In the remaining part of this section we give a general lemma about $\sigma$-girth conditions, and then explain how to derive Theorem 1 stated in the introduction from Theorem 31 
A light region $R$ is said to be connected (resp. simply connected) if the union of the faces in $R$, and the edges and vertices strictly inside $R$ is a connected (resp. simply connected) subset of the sphere. For instance, the light region in Figure 21 is simply connected. When we consider the simple connectedness of a light region containing the outer face, we think of the outer face simply as a marked face of a hypermap on the sphere, so that this face is finite and simply connected.

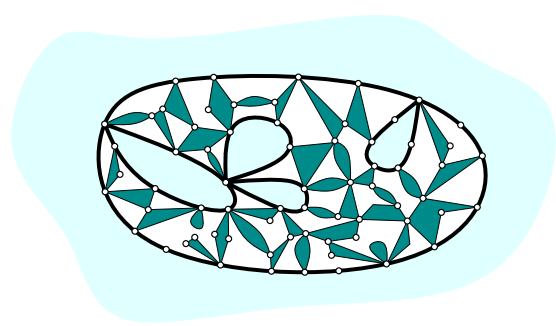

Figure 21. A simply connected light region.

The following lemma shows that the $\sigma$-girth condition can be stated as a condition on simply connected light regions whenever $\sigma_{\text {total }}=0$ (hence in particular when $\sigma$ is fitting).

Lemma 32. Let $H$ be a dark-rooted, light-rooted or vertex-rooted hypermap, and let $\sigma$ be a charge function such that $\sigma_{\text {total }}=0$. The hypermap satisfies the $\sigma$-girth condition if and only if the inequalities and strict inequalities stated in Definition 23 hold for every simply connected light region $R$.

Remark 33. We point out that, in general (even if $\sigma_{\text {total }}=0$ ), the $\sigma$-girth condition might not be satisfied even if the inequalities and strict inequalities stated in Definition 23 hold for every light region $R$ whose boundary is a simple cycle.

Proof. We treat the case in which $H$ is dark-rooted (the cases of light-rooted and vertexrooted hypermaps are proved similarly). We suppose that any simply connected light region $R$ satisfies $|\partial R| \geq \sigma(R)$ with strict inequality if all of the outer vertices are strictly inside $R$. We want to prove that the same property holds for any light region $R$. Suppose this does not hold, and take $R_{0}$ a light region such that the property does not hold and $\left|\partial R_{0}\right|$ is minimal. If $R_{0}$ is not connected, then $R_{0}$ is the disjoint union of two light regions $R_{1}$ and $R_{2}$ (with $\left|\partial R_{1}\right| \geq 1$ and $\partial R_{2} \mid \geq 1$ ). We have $\left|\partial R_{0}\right|=\left|\partial R_{1}\right|+\left|\partial R_{2}\right|$ and $\sigma\left(R_{0}\right)=\sigma\left(R_{1}\right)+\sigma\left(R_{2}\right)$ which contradicts the minimality of $\left|\partial R_{0}\right|$ (note that if the outer vertices are strictly inside $R_{0}$, then they are strictly inside either $R_{1}$ or $R_{2}$ ). Now if $R_{0}$ is connected but not simply connected, then $R_{0}$ is the intersection of two light regions $R_{1}$ and $R_{2}$ such that every face of $H$ is inside $R_{1}$ or $R_{2}$ and every vertex of $H$ is strictly inside $R_{1}$ or $R_{2}$. Hence, $\sigma\left(R_{0}\right)=\sigma\left(R_{1}\right)+\sigma\left(R_{2}\right)-\sigma_{\text {total }}=\sigma\left(R_{1}\right)+\sigma\left(R_{2}\right)$. Moreover, $\partial R_{0}$ is the disjoint union of $\partial R_{1}$ and $\partial R_{2}$. Thus $\left|\partial R_{0}\right|=\left|\partial R_{1}\right|+\left|\partial R_{2}\right|$ and this again contradicts the minimality of $\left|\partial R_{0}\right|$ (note that if the outer vertices are strictly inside $R_{0}$, then they are strictly inside both $R_{1}$ and $R_{2}$ ). Thus $R_{0}$ must be simply connected, which is a contradiction.

We now explain how to get Theorem 1 from Theorem 31 . Recall that vertex-rooted maps identify with vertex-rooted hypermaps such that every dark face has degree 2 (see Figure 1). We can therefore translate the setting of 1 in terms of hypermaps. Let $\mathcal{C}$ be the set of pairs $(H, \sigma)$ such that $H$ is a vertex-rooted hypermap where every dark face has degree 2 , and $\sigma$ is a fitting charge function with $\sigma(e)=-2$ for every dark face $e$ and $\sigma(f)=2$ for every light face $f$. We need to prove the two following claims:

Claim 34. The set $\mathcal{C}$ identifies to the set of partially charged maps considered in Theorem 1 . 
Claim 35. The weighted hypermobiles associated with the set $\mathcal{C}$ by the bijection of Theorem 31 identify with the suitably weighted mobiles considered in Theorem 1.

We first prove Claim 34. If $M$ is a vertex-rooted map endowed with a partial charge function $\sigma$, we let $H$ be the vertex-rooted hypermap identified to $M$, keeping the same $\sigma$-values at vertices, and setting $\sigma(e)=-2$ for every dark face $e$ (of degree 2, corresponding to an edge of $M$ ) and $\sigma(f)=2$ for every light face $f$ (corresponding to a face of $M$ ). Note that Condition (b) for a partial charge function $\sigma$ gives $\sigma_{\text {total }}=0$ (by the Euler relation). Thus, proving Claim 34 amounts to proving that Condition (a) holds for $\sigma$ if and only if $M$ satisfies the $\sigma$-girth condition. If $R$ is a set of faces of the map $M$, we consider the set $E(R)$ of edges of $M$ having both incident faces in $R$. Thus $\bar{R}=R \cup E(R)$ identifies to a light region of $H$, and it is easily seen that if $\bar{R}$ is simply connected then the Euler relation gives

$$
\sigma(\bar{R})=2|R|-2|E(R)|+\sum_{v \text { inside } R} \sigma(v)=2+\sum_{v \text { inside } R}(\sigma(v)-2)
$$

Therefore Condition (a) for a partial charge function $\sigma$ can be reformulated as: "for any simply connected light region of $M$ of the form $\bar{R}=R \cup E(R),|\partial \bar{R}| \leq \sigma(\bar{R})$ with strict inequality if the root-vertex $v_{0}$ is inside $\bar{R}$ ". Moreover it is easy to check that if $\bar{R}^{\prime}=R \cup E^{\prime}$ with $E^{\prime} \subseteq E(R)$, then $\left|\partial \bar{R}^{\prime}\right|-\sigma\left(\bar{R}^{\prime}\right) \leq|\partial \bar{R}|-\sigma(\bar{R})$. Thus Condition (a) is equivalent to $|\partial \bar{R}| \leq \sigma(\bar{R})$ (with strict inequality if $v_{0}$ inside $\bar{R}$ ) for any light simply connected region $\bar{R}$ of $M$. This together with Lemma 32 proves Claim 34.

It only remains to prove Claim 35. First note that the hypermobiles associated with maps have all the dark square vertices of degree 2, hence (upon removing the dark square vertices) these hypermobiles identify with the mobiles as defined in the introduction. Hence by Theorem 31 the weighted hypermobiles associated with the set $\mathcal{C}$ are mobiles having excess 0 , with weights on half-edges such that

- every half-edge has a positive weight if it is incident to a round vertex and has a non-positive weight otherwise,

- for every edge $e$, the weights of the two half-edges of $e$ add up to 0 ,

- every light square vertex $v$ has weight $2-\operatorname{deg}(v)$.

These mobiles clearly identify with the suitably weighted mobiles considered in Theorem 1 upon replacing the weights on half-edges (summing to 0) by non-negative weights on edges. This proves Claim 35 and Theorem 1 .

\section{ApPliCATIONS OF THE BIJECTION FOR CHARGED HYPERMAPS TO ANNULAR HYPERMAPS}

In this section we characterize the $\sigma$-girth condition for particular choices of the charge function $\sigma$, and derive from it bijections for annular hypermaps.

Given a real number $d$, we define the charge function $\sigma_{d}$ by

- $\sigma_{d}(v)=d$ for every vertex $v$,

- $\sigma_{d}(f)=d$ for every light face $f$,

- $\sigma_{d}(f)=d-d \cdot \operatorname{deg}(f)$ for every dark face.

Lemma 36. For any simply-connected light region $R, \sigma_{d}(R)=d$. Moreover $\sigma_{d \text { total }}=2 d$.

Proof. Let $V, E, F, K$ be respectively the set of vertices strictly inside $R$, edges strictly inside $R$, light faces inside $R$, and dark faces inside $R$. By the Euler relation we get,

$$
\sigma_{d}(R)=d(|V|+|F|+|K|-|E|)=d,
$$

because $R$ is simply connected. Similarly, the Euler relation gives $\sigma_{d \text { total }}=2 d$.

We now define a charge function, which will make clear that Theorem 7 is a special case of Theorem 25. Observe that an inward cycle (as defined in Section 3) is the boundary $C=\partial R$ of a simply connected light region $R$ not containing the outer face, such that $C$ is a simple cycle. 
Lemma 37. Let $d$ be a positive integer and let $H$ be a dark-rooted hypermap of outer degree d. Let $\sigma$ be the charge function defined by

- $\sigma(v)=d$ for every inner vertex $v$, and $\sigma(v)=0$ for every outer vertex $v$,

- $\sigma(f)=d$ for every light face $f$,

- $\sigma(f)=d-d \cdot \operatorname{deg}(f)$ for every inner dark face, and $\sigma_{d}\left(f_{0}\right)=-d$ for the dark outer face $f_{0}$.

The hypermap $H$ satisfies the $\sigma$-girth condition if and only if $H$ has ingirth $d$ (i.e., every inward cycle $C$ of $H$ has length at least d). Moreover in this case, the outer face is simple, and $\sigma_{\text {total }}=0$.

Let $\sigma$ be the charge function of Lemma 37. It is clear that the definition of $d$-weighted hyperorientations coincide with the definition of $\sigma$-weighted hyperorientations. Moreover Lemma 37 together with Theorem 25 implies that $H$ admits a (unique) $\sigma$-weighted hyperorientation in $\mathcal{H}_{-}$if and only if it has ingirth at least $d$. Thus Theorem 7 is a special case of Theorem 25

Instead of proving Lemma 37, we will prove a slight extension which will be used for counting hypermaps with given ingirth in Section 8. We define an annular hypermap as a face-rooted hypermap with a marked inner face (hence $H$ has two distinct marked faces). Let $H$ be an annular hypermap, let $f_{0}$ be its outer face $f_{0}$, and let $f_{1}$ be its marked inner face. The separating ingirth of $H$ is the minimal length of the boundary of a light region containing $f_{1}$ but not $f_{0}$. Observe that this minimal length is necessarily achieved for a boundary $C$ which is a simple cycle, that is, an inward cycle containing $f_{1}$. We call separating outgirth of $H$ the minimal length of the boundary of a light region containing $f_{0}$ but not $f_{1}$. This minimal length is necessarily achieved for a boundary $C$ which is a simple cycle. We call separating outward cycle a simple cycle which is the boundary of a light region containing $f_{0}$ but not $f_{1}$ (so that the separating outgirth is the minimal length of separating outward cycles). We call non-separating ingirth of $H$ the minimal length of the boundary of a simply connected light region containing neither $f_{0}$ nor $f_{1}$. Observe that this minimal length is not necessarily achieved for a boundary $C$ which is simple (it could be that $C$ is the union of two simple cycles).

Lemma 38. Let d,e be positive integers. Let $H$ be an annular hypermap with a dark outer face $f_{0}$ of degree $e$ and a marked inner face $f_{1}$. We consider the charge function $\sigma$ defined by

- $\sigma(v)=d$ for every inner vertex $v$, and $\sigma(v)=0$ for every outer vertex $v$,

- $\sigma(f)=d$ for every non-marked light face $f$,

- $\sigma(f)=d-d \cdot \operatorname{deg}(f)$ for every non-marked inner dark face, and $\sigma\left(f_{0}\right)=-e$ for the outer face $f_{0}$,

- $\sigma\left(f_{1}\right)=e$ if the marked inner face $f_{1}$ is light, and $\sigma\left(f_{1}\right)=e-d \cdot \operatorname{deg}\left(f_{1}\right)$ if $f_{1}$ is dark.

The hypermap $H$ satisfies the $\sigma$-girth condition if and only if $H$ has non-separating ingirth at least $d$, and separating ingirth $e$. In this case $\sigma_{\text {total }}=0$ and the outer face is simple.

Observe that Lemma 37 corresponds to the special case $e=d$ of Lemma 38 (up to forgetting the marked face which plays no particular role).

Proof. Let $\sigma^{\prime}$ be the charge function defined by $\sigma^{\prime}\left(f_{0}\right)=d e-d-e, \sigma^{\prime}\left(f_{1}\right)=-d+e$, $\sigma^{\prime}(v)=-d$ if $v$ is an outer vertex, and $\sigma^{\prime}(a)=0$ for any inner vertex and any non-marked non-root face $a$. We have $\sigma=\sigma_{d}+\sigma^{\prime}$. Hence by Lemma 36, we get $\sigma(R)=d+\sigma^{\prime}(R)$ for any simply connected light region $R$. Note also that if $H$ has separating ingirth $e$, then it implies that the outer face is simple. In this case, there are $e$ outer vertices hence $\sigma_{\text {total }}^{\prime}=-2 d$, and since by Lemma $36 \sigma_{d \text { total }}=2 d$ we get $\sigma_{\text {total }}=0$.

Now assume that $H$ satisfies the $\sigma$-girth condition. For any separating inward cycle $C$, we consider the corresponding light region $R$ and get $|C| \geq \sigma(R)=d+\sigma^{\prime}(R)=d+\sigma^{\prime}\left(f_{1}\right)=e$. 
Similarly and for any non-separating inward cycle $C$ we get $|C| \geq \sigma(R)=d+\sigma^{\prime}(R)=d$. Thus $H$ has non-separating ingirth at least $d$, and separating ingirth $e$.

Conversely assume that $H$ has non-separating ingirth at least $d$, and separating ingirth $e$. We want to prove that $H$ satisfies the $\sigma$-girth condition. Since $\sigma_{\text {total }}=0$, Lemma 32 implies that we can focus on simply connected light regions of $H$. For a simply connected light region $R$, we know $\sigma(R)=d+\sigma^{\prime}(R)$, and need to prove $|\partial R|>\sigma(R)$. First suppose that $R$ does not contain $f_{0}$. We get $\sigma(R)=e$ if $f_{1} \in R$ and $\sigma(R)=d$ otherwise. Moreover $\partial R$ contains an inward cycle $C$, thus by hypothesis, $|\partial R| \geq|C| \geq \sigma(R)$. Suppose now that $R$ contains $f_{0}$. Let $b$ be the number of outer vertices incident to $\partial R$ (the other outer vertices are all strictly inside $R$ ). We get $\sigma(R)=b d-d$ if $f_{1} \in R$ and $\sigma(R)=b d-e$ otherwise. If $b=0$ then $\sigma(R)<0$, hence $|\partial R|>\sigma(R)$ holds trivially. Suppose now that $b>0$. In this case the light region $R^{\prime}=R \backslash\left\{f_{0}\right\}$ is the disjoint union of $b$ simply connected light regions $R_{1}, \ldots, R_{b}$, and $\partial R^{\prime}$ is the disjoint union of their boundaries $\partial R_{1}, \ldots, \partial R_{b}$. Each boundary $\partial R_{i}$ contains an inward cycle, so $\left|\partial R_{i}\right| \geq e$ if $R_{i}$ contains $f_{1}$ and $\left|\partial R_{i}\right| \geq d$ otherwise. Since $|\partial R|=\left|\partial R^{\prime}\right|-e=\sum_{i=1}^{b}\left|\partial R_{i}\right|-e$ we get $|\partial R| \geq b d-d=\sigma(R)$ if $R$ contains $f_{1}$ and $|\partial R| \geq b d-e=\sigma(R)$ otherwise. This completes the proof that $H$ satisfies the $\sigma$-girth condition.

We now give a similar result for light-rooted hypermaps.

Lemma 39. Let $d, e$ be positive integers. Let $H$ be an annular hypermap with a light outer face face $f_{0}$ of degree $e$ and a marked inner face $f_{1}$. We consider the charge function $\sigma$ defined by

- $\sigma(v)=d$ for every vertex $v$,

- $\sigma(f)=d$ for every non-marked inner light face $f$, and $\sigma\left(f_{0}\right)=e$ for the outer face $f_{0}$,

- $\sigma(f)=d-d \cdot \operatorname{deg}(f)$ for every non-marked inner dark face,

- $\sigma\left(f_{1}\right)=-e$ if the marked face $f_{1}$ is light, and $\sigma\left(f_{1}\right)=-e-d \cdot \operatorname{deg}\left(f_{1}\right)$ if $f_{1}$ is dark.

Then $\sigma_{\text {total }}=0$, and the hypermap $H$ satisfies the $\sigma$-girth condition if and only if $H$ has non-separating ingirth at least $d$, and separating outgirth e and such that the only outward cycle of length $e$ is the contour of the outer face.

Proof. We have $\sigma=\sigma_{d}+\sigma^{\prime}$, where $\sigma^{\prime}\left(f_{0}\right)=-d+e, \sigma^{\prime}\left(f_{1}\right)=-d-e$, and $\sigma^{\prime}(a)=0$ for any vertex and any non-marked non-root face $a$. Lemma 36 gives $\sigma_{\text {total }}=\sigma_{d \text { total }}+\sigma^{\prime}\left(f_{0}\right)+$ $\sigma^{\prime}\left(f_{1}\right)=0$. Using Lemmas 32 and 36 , we easily see that the $\sigma$-girth condition translates into the following condition for any simply connected region $R$ :

(i) $|\partial R| \geq d$ if $R$ contains neither $f_{0}$ nor $f_{1}$,

(ii) $|\partial R| \geq e$ if $R$ contains $f_{0}$ but not $f_{1}$, with strict inequality if $R \neq\left\{f_{0}\right\}$,

(iii) $|\partial R| \geq-e$ if $R$ contains $f_{1}$ but not $f_{0}$,

(iv) $|\partial R|>-d$ if $R$ contains both $f_{0}$ and $f_{1}$,

The conditions (iii) and (iv) are void, while the conditions (i) and (ii) are clearly equivalent to the fact that $H$ has non-separating ingirth at least $d$, separating outgirth $e$, and the contour of the outer face is the only separating outward cycle of length $e$.

We will now use Lemmas 38 and 39 in conjunction with Theorems 29 and 30 to get bijections with classes of hypermobiles. For any integers $d, e$ (where $e$ is allowed to be negative), we call $(d, e)$-weighted hypermobile a consistently weighted hypermobile (that is, a weighted hypermobile such that edges incident to a round vertex have positive weight, while edges incident to a light square vertex have non-positive weight), with a marked square vertex, such that

- every round vertex has weight $d$.

- every unmarked light square vertex $v$ has weight $d-\operatorname{deg}(v)$,

- every unmarked dark square vertex $v$ has weight $d \cdot \operatorname{deg}(v)-d-\operatorname{deg}(v)$, 

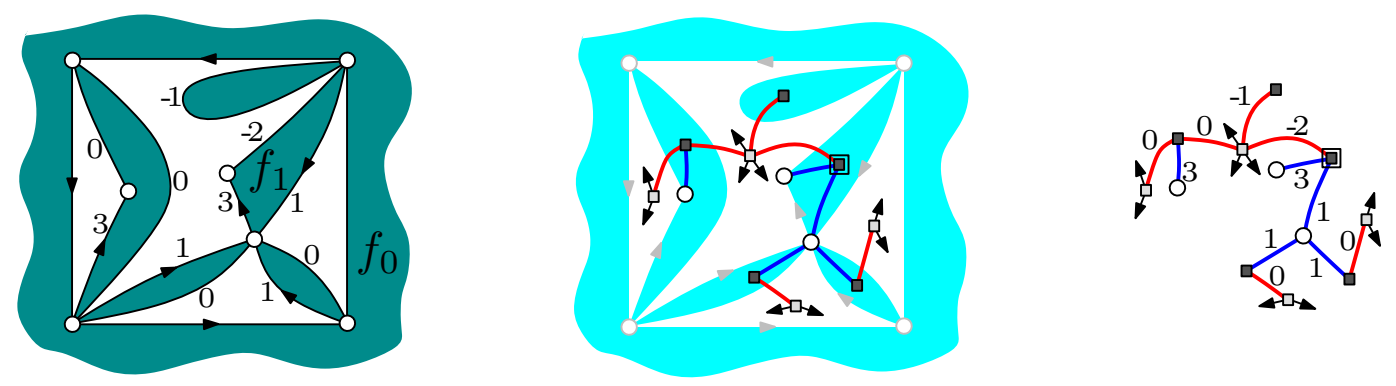

Figure 22. The bijection of Theorem 40 on an example (case $d=3, e=4$, with a dark marked inner face $f_{1}$ ).

- the marked square vertex $v$ has weight $e-\operatorname{deg}(v)$ if $v$ is light and $d \cdot \operatorname{deg}(v)-e-\operatorname{deg}(v)$ if $v$ is dark.

By similar arguments as in Claim 8 one can check that a $(d, e)$-weighted hypermobile always has excess $-e$.

Let $\mathcal{B}^{(d, e)}$ be the family of annular hypermaps with a dark outer face of degree $e$, nonseparating ingirth at least $d$ and separating ingirth $e$. Let $\mathcal{B}^{\prime(d, e)}$ be the set of fittingly charged annular hypermaps $(H, \sigma)$ with a dark outer face of degree $e$, where the charge $\sigma$ is defined as in Lemma 38. By Lemma 38 we can identify the sets $\mathcal{B}^{(d, e)}$ and $\mathcal{B}^{\prime(d, e)}$. Moreover, by Theorem 29 the mapping $\Phi_{-}$gives a bijection between $\mathcal{B}^{\prime(d, e)}$ and the family of $(d, e)$-weighted hypermobiles. Thus we obtain the following result (see Figure 22 for an example).

Theorem 40. For $d$ and e positive integers, the family $\mathcal{B}^{(d, e)}$ is in bijection with the family of $(d, e)$-weighted hypermobiles. Each light (resp. dark) inner face in the hypermap corresponds to a light (resp. dark) square vertex of the same degree in the associated hypermobile. Moreover, the marked inner face corresponds to the marked square vertex.

Remark 41. Theorem 40 generalizes Theorem 7 which corresponds to the case $e=d$ (by forgetting the marked inner face). Indeed the ingirth of a hypermap with a marked inner face is the minimum of its separating and non-separating ingirths. This theorem also generalize the bijection established for so-called annular maps (planar maps with a root-face and an additional marked face) in [5].

Remark 42. It would be possible to extend Theorem 40 to the case where there is a marked inner vertex $v_{1}$ instead of a marked inner face $f_{1}$. In that case the separating ingirth is the minimal boundary-length of a light region not containing $f_{0}$ but containing $v_{1}$ in its strict interior. In the associated hypermobiles, the marked vertex would be round instead of square, and its weight would be $e$ instead of $d$. It would also be possible to extend Theorem 40 to the case where there is a root-vertex $v_{0}$ instead of a root-face $f_{0}$ : this would correspond to the case $e=0$ (no constraint on the separating ingirth) and we would apply $\Phi_{0}$ instead of $\Phi_{-}$. These extensions can be obtained from the charged-map setup in a way similar to the results proved in this section.

Similarly let $\mathcal{C}^{(d, e)}$ be the set of annular hypermaps with a light outer face of degree $e$, non-separating ingirth at least $d$, separating outgirth $e$, and such that the only outward cycle of length $e$ is the contour of the outer face. Let $\mathcal{C}^{\prime(d, e)}$ be the set of fittingly charged annular hypermaps $(H, \sigma)$ with a light outer face of degree $e$, where the charge $\sigma$ is defined as in Lemma 39. By Lemma 39 we can identify the sets $\mathcal{C}^{(d, e)}$ and $\mathcal{C}^{\prime(d, e)}$, and by Theorem 30 , the mapping $\Phi_{+}$gives a bijection between $\mathcal{C}^{\prime(d, e)}$ and the family of $(d,-e)$-weighted hypermobiles. In conclusion we obtain (see Figure 23 for an example): 

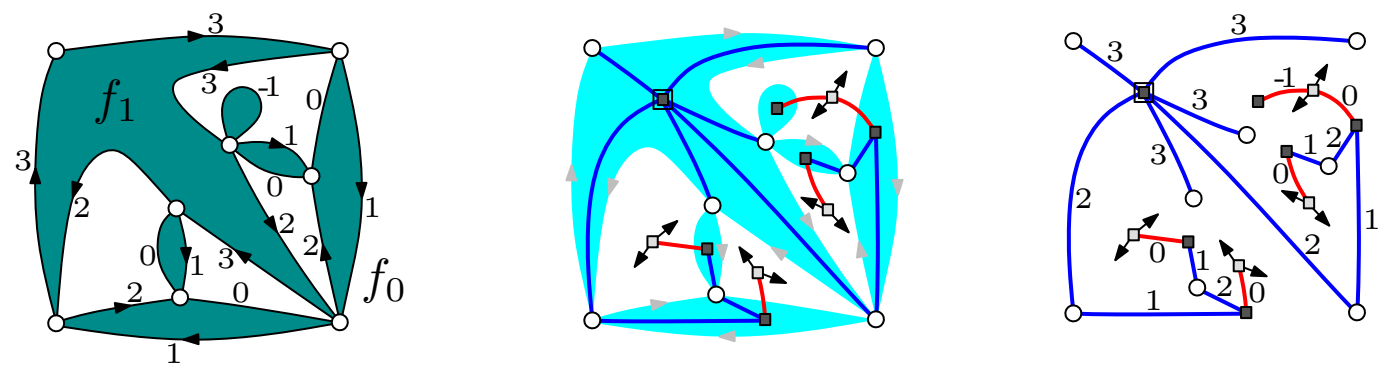

Figure 23. The bijection of Theorem 43 on an example (case $d=3, e=4$, with a dark marked inner face $f_{1}$ ).

Theorem 43. For $d$ and e positive integers, the family $\mathcal{C}^{(d, e)}$ is in bijection with the family of $(d,-e)$-weighted hypermobiles. Each light (resp. dark) inner face in the hypermap corresponds to a light (resp. dark) square vertex of the same degree in the associated hypermobile. Moreover, the marked inner face corresponds to the marked square vertex.

\section{Counting annular hypermaps ACCORDing to GiRTh PARAmeters}

In Section 4 we have counted plane hypermaps with control on the ingirth and the face degrees, but with the restriction that the outer degree is equal to the ingirth. Here we will drop this restriction. Our strategy (as in our previous article [5] dealing with maps) is to consider annular hypermaps instead of plane hypermaps, and use a canonical decomposition of annular hypermaps into two hypermaps that can be counted bijectively.

Recall that an annular hypermap is a hypermap with two marked faces: one called the outer face and the other called the marked inner face. An annular hypermap is corner-rooted by marking a corner in the outer face and a corner in the marked inner face. Let $\overrightarrow{\mathcal{A}}_{d, e}$ be the family of corner-rooted annular hypermaps of separating girth $e$ and non-separating ingirth at least $d$. Let $\overrightarrow{\mathcal{B}}_{d, e}$ (resp. $\overrightarrow{\mathcal{C}}_{d, e}$ ) be the family of corner-rooted annular hypermaps such that the underlying (unrooted) annular hypermap is in the family $\mathcal{B}_{d, e}$ (resp. $\mathcal{C}_{d, e}$ ) defined in Section 7 .

Lemma 44. There is an e-to-1 correspondence between $\overrightarrow{\mathcal{A}}_{d, e}$ and the Cartesian product $\overrightarrow{\mathcal{C}}_{d, e} \times \overrightarrow{\mathcal{B}}_{d, e}$.

Proof. We will first define the canonical cycle of an annular hypermap $H \in \overrightarrow{\mathcal{A}}_{d, e}$. For any cycles $C_{1}, C_{2}$ that are contours of some light regions $R_{1}, R_{2}$ of a hypermap $H$, we denote by $\cap\left(C_{1}, C_{2}\right)$ (resp. $\left.\cup\left(C_{1}, C_{2}\right)\right)$ the contour of the light region $R_{1} \cap R_{2}$ (resp. $R_{1} \cup R_{2}$ ). It is easy to see that

$$
\left|\cup\left(C_{1}, C_{2}\right)\right|+\left|\cap\left(C_{1}, C_{2}\right)\right|=\left|C_{1}\right|+\left|C_{2}\right| .
$$

Thus, if $C_{1}$ and $C_{2}$ are separating inward cycles of length $e$, then $\cup\left(C_{1}, C_{2}\right)$ and $\cap\left(C_{1}, C_{2}\right)$ are both separating inward cycles of length $e$ (since $H$ has separating girth $e$ ). Thus $H$ has a separating inward cycle $C$ of length $e$ which is the outermost (that is, its light region contains the light region of any separating inward cycle of length $e$ ), and we call it the canonical cycle of $H$.

We now define the $e$-to-1 correspondence between $\overrightarrow{\mathcal{A}}_{d, e}$ and $\overrightarrow{\mathcal{C}}_{d, e} \times \overrightarrow{\mathcal{B}}_{d, e}$. Let $\overrightarrow{\mathcal{A}}_{d, e}^{\bullet}$ be the set of pairs $(H, v)$ where $H \in \overrightarrow{\mathcal{A}}_{d, e}$ and $v$ is a vertex on the canonical cycle. For $(H, v) \in \overrightarrow{\mathcal{A}}_{d, e}$, with $f_{0}$ the outer face and $f_{1}$ the marked inner face of $H$, we denote by $\phi(H, v)$ the pair $(I, J)$ of corner-rooted annular hypermaps obtained by cutting along $C$ : the marked inner face of $I$ is $f_{0}$ and the outer face of $I$ is delimited by $C$, while the marked inner face of $J$ is $f_{1}$ and the outer face of $J$ is delimited by $C$ (the marked corners in the faces delimited by $C$ are at $v$ ). It is immediate to check that $I \in \overrightarrow{\mathcal{C}}_{d, e}$, and $J \in \overrightarrow{\mathcal{B}}_{d, e}$. Hence $\phi$ is a mapping from $\overrightarrow{\mathcal{A}_{d, e}^{\bullet}}$ to $\overrightarrow{\mathcal{C}}_{d, e} \times \overrightarrow{\mathcal{B}}_{d, e}$. 
It remains to prove that $\phi$ is a bijection, which we do by exhibiting the inverse mapping. For $(I, J) \in \overrightarrow{\mathcal{C}}_{d, e} \times \overrightarrow{\mathcal{B}}_{d, e}$, we let $\psi(I, J)$ be the pair $(H, v)$, where $H$ is the corner-rooted annular hypermap obtained by patching the outer face of $I$ with the outer face of $J$ so that their marked outer corners coincide, defining $v$ as their common incident vertex after patching, and defining the outer face of $H$ as the marked inner face of $I$. It is clear that $\psi \circ \phi=\mathrm{Id}$ and we need to prove $\phi \circ \psi=\mathrm{Id}$. Hence, we need to prove that if $(H, v)=\psi(I, J)$ then $H \in \overrightarrow{\mathcal{A}}_{d, e}$ and the cycle $C^{\prime}$ of $H$ resulting from merging the outer face of $I$ with the outer face of $J$ is the canonical cycle $C$ of $H$. Note that $\left|C^{\prime}\right|=e$ and $|C| \leq e$. Moreover, since $\cap\left(C, C^{\prime}\right)$ is a separating inward cycle of $J$, we get $\left|\cap\left(C, C^{\prime}\right)\right| \geq e$. And since $\cup\left(C, C^{\prime}\right)$ is a separating outward cycle of $I$, we get $\left|\cup\left(C, C^{\prime}\right)\right| \geq e$ with equality if and only if $\cup\left(C, C^{\prime}\right)=C^{\prime}$. Thus $|C|+\left|C^{\prime}\right|=\left|\cup\left(C, C^{\prime}\right)\right|+\left|\cup\left(C, C^{\prime}\right)\right| \geq 2 e$, and finally $|C|=\left|C^{\prime}\right|=e$. This implies that the separating ingirth of $H$ is $e$, and moreover $\cup\left(C, C^{\prime}\right)=C^{\prime}$ which implies that $C^{\prime}=C$ (since $C$ is the outermost separating inward cycle of length $e$ ). It only remains to show that $H$ has non-separating girth at least $d$. Let $\hat{R}$ be a light region of $H$ not containing the inner marked face $f_{1}$ and let $\hat{C}$ be the contour of $\hat{R}$. If $\cap(C, \hat{C})$ encloses no face, then $\hat{C}$ completely belongs to $I$, so that $|\hat{C}| \geq d$. Otherwise, $\cap(C, \hat{C})$ completely belongs to $J$ and is the contour of a non-empty light region not containing $f_{1}$, hence $|\cap(C, \hat{C})| \geq d$. Moreover $\cup(C, \hat{C})$ is a separating outward cycle of $I$, hence $|\cup(C, \hat{C})| \geq e$. Thus $|\hat{C}|=|\cup(C, \hat{C})|+|\cap(C, \hat{C})|-|C| \geq d$. Thus $H$ has non-separating ingirth $d$ and $H \in \overrightarrow{\mathcal{A}}_{d, e}$, which completes the proof that $\phi$ is a bijection.

For $k, \ell \geq 1$, we define $\overrightarrow{\mathcal{A}}_{d, e}^{k, \diamond \ell}$ as the family of corner-rooted annular hypermaps of separating ingirth $e$, non-separating ingirth at least $d$, where the outer face is dark of degree $k$ and the marked inner face is light of degree $\ell$. Let $A_{d, e}^{\diamond, \diamond \ell} \equiv A_{d, e}^{\diamond, \diamond \ell}\left(x_{1}, x_{2}, \ldots ; y_{1}, y_{2}, \ldots\right)$ be the generating function of $\overrightarrow{\mathcal{A}}_{d, e} k, \diamond \ell$ where $x_{i}$ and $y_{i}$ mark respectively the number of unmarked inner light and dark faces of degree $i$. We define the families $\overrightarrow{\mathcal{A}}_{d, e}^{k, \ell}, \overrightarrow{\mathcal{A}}_{d, e}^{\diamond k, \ell}$, $\overrightarrow{\mathcal{A}}_{d, e}^{\diamond k, \diamond \ell}$ (depending on the types, light or dark, of the outer face and of the marked inner face) and their associated generating functions similarly. Let $\overrightarrow{\mathcal{B}} d, k$ (resp. $\overrightarrow{\mathcal{C}} \overrightarrow{d, e}$ ) be the subfamily of $\overrightarrow{\mathcal{B}}_{d, e}$ (resp. $\overrightarrow{\mathcal{C}}_{d, e}$ ) for which the marked inner face is dark of degree $k$. Let $B_{d, e}^{k} \equiv B_{d, e}^{k}\left(x_{1}, x_{2}, \ldots ; y_{1}, y_{2}, \ldots\right)\left(\right.$ resp. $\left.C_{d, e}^{k} \equiv C_{d, e}^{\star k}\left(x_{1}, x_{2}, \ldots ; y_{1}, y_{2}, \ldots\right)\right)$ be the generating function of $\overrightarrow{\mathcal{B}}_{d, e}^{k}$ (resp. $\overrightarrow{\mathcal{C}}_{d, e}^{k}$ ) where $x_{i}$ and $y_{i}$ mark respectively the number of light and dark unmarked inner faces of degree $i$. We define the families $\overrightarrow{\mathcal{B}}_{d, e}^{\diamond k}, \overrightarrow{\mathcal{C}}_{d, e}^{\diamond k}$ and their generating functions similarly. Lemma 44 gives

$$
A_{d, e}^{* k, \star \ell}=\frac{1}{e} C_{d, e}^{* k} B_{d, e}^{\star \ell} .
$$

for $* \in\{\diamond, \diamond\}$ and $\star \in\{\bullet, \diamond\}$.

We now use Theorems 40 and 43 to determine $B_{d, e}^{\star \ell}$ and $C_{d, e}^{* k}$. Theorem 40 gives a bijection between $\mathcal{B}_{d, e}$ and the family of $(d, e)$-weighted hypermobiles. It is easily seen that marking a corner in the marked inner face of an annular hypermap in $\mathcal{B}_{d, e}$ corresponds to marking a corner at the marked square vertex of the associated $(d, e)$-weighted hypermobile. Thus, there is an $e$-to- 1 correspondence between $\overrightarrow{\mathcal{B}}_{d, e}$ and the family $\mathcal{T}_{d, e}$ of $(d, e)$-weighted hypermobiles with a marked corner at the marked square vertex (the factor $e$ correspond to choosing the marked corner in the outer face of the annular hypermap). Moreover the degree and color of the marked inner face of the hypermap corresponds to the degree and color of the marked vertex of the hypermobile. Thus by decomposing hypermobiles in $\mathcal{T}_{d, e}$ at their root-vertex (which yields a sequence of planted $d$-hypermobiles) we get

$$
B_{d, e}^{\diamond k}=e\left[u^{e}\right] W(u)^{k}, \quad B_{d, e}^{k}=e\left[u^{-e}\right] L(u)^{k},
$$

where $W(u)$ and $L(u)$ are defined by (1). Similarly, Theorem 43 leads to

$$
C_{d, e}^{\diamond k}=e\left[u^{-e}\right] W(u)^{k}, \quad C_{d, e}^{k}=e\left[u^{e}\right] L(u)^{k} .
$$


We therefore obtain the following result.

Theorem 45. For $e, d, k, \ell \geq 1$, the generating functions of corner-rooted annular maps have the following expressions:

$$
\begin{aligned}
A_{d, e}^{\diamond k, \diamond \ell} & =e\left[u^{e}\right] L(u)^{k}\left[v^{e}\right] W(v)^{\ell}, & A_{d, e}^{\diamond k, \bullet} & =e\left[u^{-e}\right] W(u)^{k}\left[v^{-e}\right] L(v)^{\ell}, \\
A_{d, e}^{\diamond k, \ell} & =e\left[u^{e}\right] L(u)^{k}\left[v^{-e}\right] L(v)^{\ell}, & A_{d, e}^{\diamond k, \diamond \ell} & =e\left[u^{-e}\right] W(u)^{k}\left[v^{e}\right] W(v)^{\ell},
\end{aligned}
$$

where $L(u)$ and $W(u)$ are specified by (1), (3) and (4).

Remark 46. Under the specialization $y_{2}=1, y_{i}=0$ for $i \neq 2$, the generating function $A_{d, e}^{\diamond k, \diamond \ell}$ counts corner-rooted annular maps with control on the separating girth, the nonseparating girth, and the face degrees. Hence Theorem 45 gives an extension to annular hypermaps of the counting results obtained in 5 for annular maps.

Moreover, it is easy to see that the generating function $F_{d}$ defined in Section 4 is related to $A_{d, d}^{\diamond d, \star \ell}$ by $\ell \frac{\partial F_{d}}{x_{\ell}}=A_{d, d}^{\diamond d, \diamond \ell}$ and $A_{d, d}^{\diamond}=\ell \frac{\partial F_{d}}{y_{\ell}}$. Hence the expressions for the derivatives of $F_{d}$ given in Theorem 12 are a special case of Theorem 45.

For any sets $\Delta, \Delta^{\prime}$, the generating function $A_{d, e, \Delta, \Delta^{\prime}}^{* k, \star \ell}$ of hypermaps in $\overrightarrow{\mathcal{A}}_{d, e, \Delta, \Delta^{\prime}}^{* k, \star \ell}$ with inner light faces having degrees in $\Delta$ and inner dark faces having degrees in $\Delta^{\prime}$ is obtained by setting $x_{i}=0$ for $i \notin \Delta, y_{i}=0$ for $i \notin \Delta^{\prime}$. This is an algebraic series as soon as $\Delta, \Delta^{\prime}$ are both finite. For instance, for $d=4, e=2, \Delta=\{4\}, \Delta^{\prime}=\{3\}$, we have

$$
A_{4,2}^{\diamond, \diamond 2}=2\left(4 L_{2}+6 L_{3}^{2}\right)\left(1+W_{0}\right)^{2},
$$

where the series $\left\{L_{0}, L_{1}, L_{2}, L_{3}, L_{4}, W_{0}, W_{1}, W_{2}, W_{3}, W_{4}\right\}$ (already considered in the example of Section 4) are specified by

$$
\begin{aligned}
& L_{0}=x_{4}\left(1+W_{0}\right)^{3}, L_{1}=W_{1}^{3}+2 W_{1} W_{2}+W_{3}, L_{2}=W_{1}^{2}+W_{2}, L_{3}=W_{1}, L_{4}=1, \\
& W_{0}=2 y_{3} L_{2} L_{3}, W_{1}=y_{3}\left(2 L_{1} L_{3}+L_{2}^{2}\right), W_{2}=2 y_{3} L_{1} L_{2}, W_{3}=y_{3} L_{1}^{2}, W_{4}=2 y_{3} L_{1} .
\end{aligned}
$$

\section{Proof of Theorems 4 and 6 about the master bijection}

In this section we prove Theorems 4 and 6 about the three master bijections $\Phi_{+}, \Phi_{-}$ and $\Phi_{0}$. The proofs for the three bijections are similar. We give a detailed proof for $\Phi_{+}$in Section 9.1 and a more succinct proof for $\Phi_{-}$and $\Phi_{0}$ in Sections 9.2 and 9.3

9.1. Proof for $\Phi_{+}$. Let $\mathcal{J}_{+}$be the family of light-rooted hyperorientations such that all outer edges are 1-way. Note that $\mathcal{H}_{+}$is a subset of $\mathcal{J}_{+}$. We now extend the definition of the mapping $\Phi_{+}$to $\mathcal{J}_{+}$. For $H \in \mathcal{J}_{+}$, we define $\Phi_{+}(H)$ as the map obtained from $H$ by placing a dark (resp. light) square vertex in each dark (resp. light) face, then applying the local rule of Figure 7 to each edge of $H$, and then deleting the edges of $H$ and the light square vertex corresponding to the outer face (see Figures 24 and 25 for examples).

Lemma 47. Let $H$ be an hyoeroriented hypermap in $\mathcal{J}_{+}$, and let $T=\Phi_{+}(H)$. Then, $T$ is a hypermobile if and only if $H \in \mathcal{H}_{+}$.

Moreover, in this case the following property holds for each inner 1-way edge e of $H$ :

$(\boldsymbol{M})$ Let $u, v$ be the square vertices in the faces incident to $e$, and let $C$ be the cycle contained in $T \cup\left\{e^{*}\right\}$, where $e^{*}$ is the edge joining $u$ and $v$ across $e$. Then $e$ is oriented from the outside of $C$ to the inside of $C$ (across $\left.e^{*}\right)$.

Proof. First observe that $T$ is a hypermobile if and only if it is a tree (since the local conditions of hypermobiles are satisfied by $T$ ). Let $N_{v}, N_{e}, N_{f}$ be the numbers of vertices, edges, and faces of $H$. The map $T$ has $E=N_{e}$ edges (because each edge of $H$ yields an edge in $T$ ), and $V=N_{v}+N_{f}-1$ vertices (the -1 accounts for the deletion of the light square vertex in the outer face of $H$ ). The Euler relation for $H$ gives $N_{v}-N_{e}+N_{f}=2$, hence $E=V-1$. Thus $T$ is a hypermobile if and only if it is acyclic. 

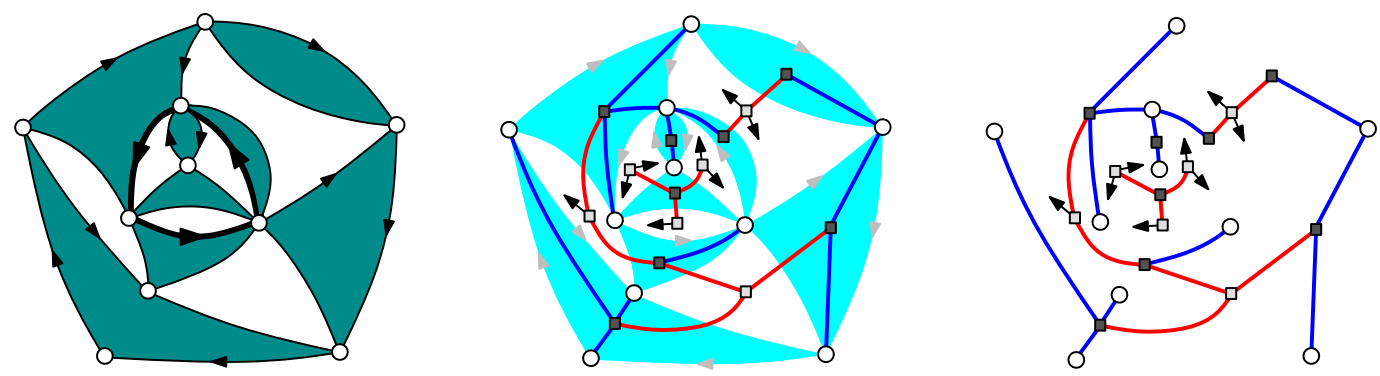

FiguRE 24. If $H$ has a counterclockwise circuit $C$ (shown in bold line on the leftmost picture), then $\Phi(H)$ has a cycle outside of $C$.
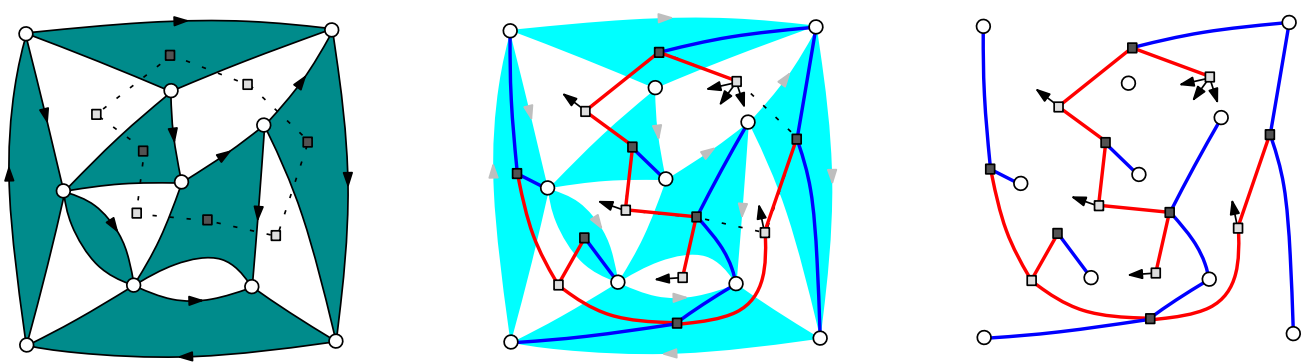

Figure 25. If $H$ is not accessible from the outer boundary, there is a cycle $C$ in the dual of $H$ such that all edges dual to edges on $C$ are either 0-way or 1-way from the inside to the outside of $C$; then $\Phi(H)$ has a cycle in the area exterior to $C$ (including $C$ ).

Now we prove that if $H \notin \mathcal{H}_{+}$, then $T$ has a cycle. For $H \notin \mathcal{H}_{+}$, either $H$ has a counterclockwise circuit or $H$ is not accessible from the outer vertices. Suppose first that $H$ has a counterclockwise circuit $C$ (see Figure 24). Let $n_{v}$ and $n_{e}$ be the numbers of vertices and edges of $H$ that are on $C$ or outside of $C$, and let $n_{f}$ be the number of faces of $H$ that are outside of $C$. Note that the Euler relation (applied to $H$ where everything strictly inside $C$ is erased) yields $n_{v}-n_{e}+n_{f}=1$. Let $K$ be the submap of $T$ made of all its vertices on $C$ or outside of $C$ and all its edges outside of $C$. Since all edges on $C$ are counterclockwise, the submap $K$ has $E=n_{e}$ edges (because each edge on $C$ yields an edge of $T$ outside of $C$ ), and $V=n_{v}+n_{f}-1$ vertices (the -1 accounts for the deletion of the light square vertex in the outer face). Hence, $E=V$, so that $K$ has a cycle, and $T$ is not a tree. Suppose now that $H$ is not accessible from the outer vertices (see Figure 25). We consider the dual map $H^{*}$ which is obtained by placing a vertex $f^{*}$ of $H^{*}$ in each face $f$ of $H$, and drawing an edge $e^{*}$ of $H^{*}$ from $f_{1}^{*}$ to $f_{2}^{*}$ across each edge $e$ of $H$ separating the faces $f_{1}$ and $f_{2}$. An outward cocycle of $H$ is a sequence $D=e_{1}, \ldots, e_{k}$ of edges such that the dual edges $D^{*}=e_{1}^{*}, \ldots, e_{k}^{*}$ form a simple cycle of $H^{*}$, and for all $i \in\{1, \ldots, k\}$ the edge $e_{i}$ is either 0 -way or 1 -way toward the outside of $D^{*}$. It is not hard to prove that because $H$ is not accessible it has an outward cocycle $D=e_{1}, \ldots, e_{k}$ (to prove the existence of $D$ start by considering the set of vertices of $H$ that are reachable from the outer vertices). Let $n_{v}^{*}, n_{e}^{*}$ be the number of vertices and edges of $H^{*}$ that are on $D^{*}$ or outside of $D^{*}$, and let $n_{f}^{*}$ be the number of faces of $H^{*}$ that are outside of $D^{*}$. By the Euler relation applied to $H^{*}$ (where everything strictly inside $D^{*}$ is erased), $n_{v}^{*}-n_{e}^{*}+n_{f}^{*}=1$. Let $K$ be the submap of $T$ made of all its vertices on $D^{*}$ or outside of $D^{*}$ and all its edges on $D^{*}$ or outside of $D^{*}$. Since all edges in $D$ are 0 -way or are 1-way from the inside to the outside of $D^{*}$, the submap $K$ has $E=n_{e}^{*}$ edges (because each edge in $D$ yields an edge of $T$ on $D^{*}$ or outside 
of $D^{*}$ ), and $V=n_{v}^{*}+n_{f}^{*}-1$ vertices (the -1 accounts for the deletion of the light square vertex in the outer face of $H$ ). Hence $E=V$, so that $K$ has a cycle, and $T$ is not a tree.

Next we prove that, if $H \in \mathcal{H}_{+}$, then $T$ is a hypermobile. We suppose by contradiction that $H \in \mathcal{H}_{+}$and $T$ has a cycle $C$. We first consider the case where all vertices on $C$ are squares. In this case, the edges dual to edges on $C$ form a cocycle of 0 -way edges, so the (non-empty) set of vertices of $H$ inside $C$ is unreachable from the outer vertices of $H$, a contradiction. We now suppose that there is a round vertex $u_{0}$ on $C$. Let $v_{0}$ be the (dark square) vertex following $u_{0}$ in clockwise order around $C$, and let $e_{0}$ be the edge of $H$ following the edge $\left\{u_{0}, v_{0}\right\}$ clockwise around $u_{0}$; see Figure 26(a) By the local rule of Figure 7, $e_{0}$ is 1-way toward $u_{0}$, and by accessibility of $H, e_{0}$ is the ending edge of some directed path $P_{0}$ starting from some outer vertex of $H$. Let $\widetilde{P_{0}}$ be the last portion of $P_{0}$ inside $C$, and let $u_{1} \in C$ be the starting vertex of $\widetilde{P_{0}}$. Note that $u_{1} \neq u_{0}$, otherwise $\widetilde{P}_{0}$ would form a counterclockwise circuit. By the same argument as for $u_{0}$, the next vertex $v_{1}$ after $u_{1}$ in clockwise order around $C$ is a dark square, and denoting by $e_{1}$ the next edge after $\left\{u_{1}, v_{1}\right\}$ in clockwise order around $u_{1}$, there is a path $\widetilde{P_{1}}$ inside $C$ that starts from a vertex $u_{2} \in C$ and ends at $e_{1}$. Note that $u_{2}$ is not on the portion of $C$ going clockwise from $u_{0}$ to $u_{1}$ (otherwise it would yield a counterclockwise cycle in $H$ ). Continuing iteratively we reach a contradiction, because at each step $i$, the vertex $u_{i}$ has to avoid a strictly growing portion of $C$; see Figure 26(a).

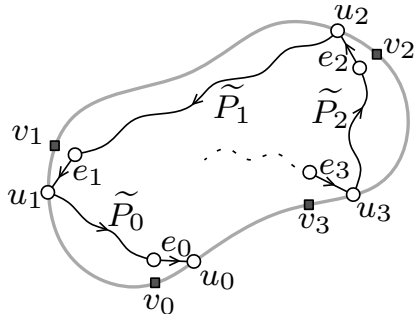

(a)

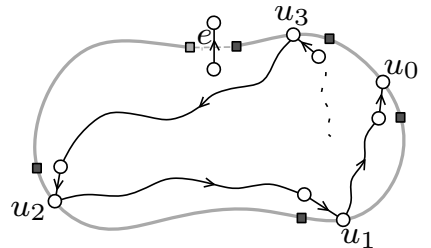

(b)

Figure 26. (a) If $H$ is accessible, then the existence of a cycle in $\Phi(H)$ implies the existence of a counterclockwise circuit in $H$. (b) Proof of the property $(\boldsymbol{A})$.

Lastly, the proof of property ( $)$ follows the exact same line of argument as above. Assuming by contradiction that $H \in \mathcal{H}_{+}$but that (\$) does not hold for an edge $e$ we consider two cases. First if all vertices of $C$ are square, then the dual of the edges of $C$ are 0way, so the inside of $C$ is unreachable from the outer vertices, giving a contradiction. Second, if there is a round vertex $u_{0} \in H$ on $C$, then one can construct a sequence $u_{0}, u_{1}, u_{2}, \ldots$ of vertices on $C$ such that $u_{i}$ has to avoid a strictly growing portion of $C$, again giving a contradiction; see Figure 26(b)

Next we prove that the mappings $\Phi_{+}$and $\Psi_{+}$are inverse bijections.

Lemma 48. Let $T$ be a hypermobile of positive excess, and let $H=\Psi_{+}(T)$ be the closure of $T$. Then $T$ is a tree covering all the vertices of $H$ and all the square vertices placed in the inner faces of $H$.

Proof. To prove the lemma, it is convenient to see the closure mapping $\Phi_{+}$as done "step by step". Let $\widehat{T}$ be the outerplanar map associated with $T$. Starting from $\widehat{T}$, define a local closure as the operation of gluing a cw-outer edge $e_{1}$ with a ccw-outer edge $e_{2}$ such that $e_{1}$ and $e_{2}$ are consecutive edges in clockwise order around the outer face; see Figure 27 Then $H$ is obtained as the result of performing local closure operations greedily until there remains no pair to glue. At each step of the closure, we call floating a vertex which is 

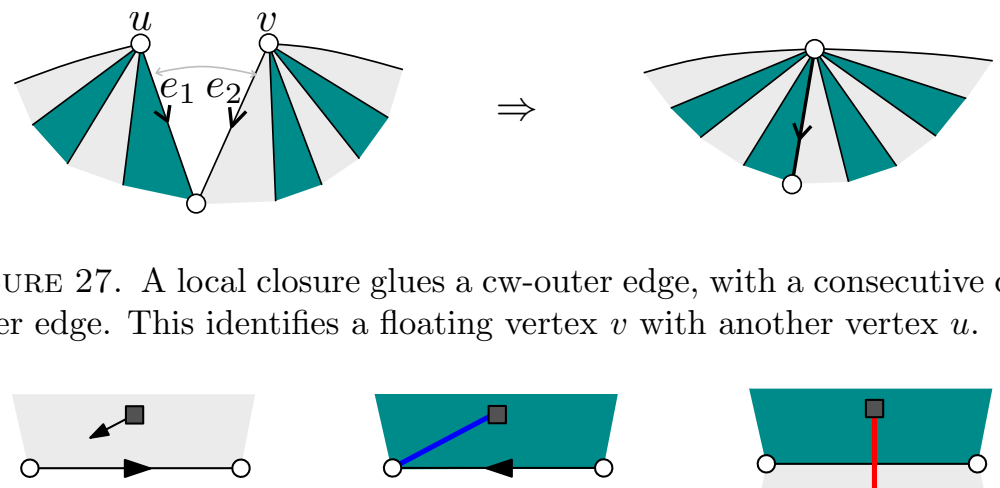

Figure 27. A local closure glues a cw-outer edge, with a consecutive ccwouter edge. This identifies a floating vertex $v$ with another vertex $u$.
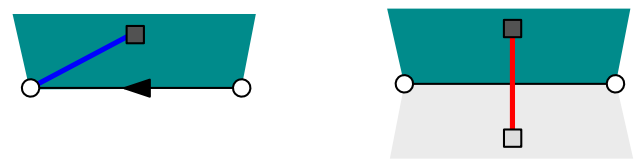

Figure 28. The local rules for the configuration of $T$ for each incidence of an inner face with an edge of $H$.

the origin of a ccw-outer edge. We now claim that at each step of the closure, $T$ is a tree covering all the vertices of the partially closed map except all the floating vertices. Indeed this property is true for $\widehat{T}$. Moreover, it remains true through local closures because each local closure identifies a floating vertex with another vertex, and the resulting vertex is floating if both vertices are floating; see Figure 27.

Corollary 49. Let $T \in \mathcal{T}_{+}$, and let $H=\Psi_{+}(T)$. Then $H$ is in $\mathcal{H}_{+}$, and $\Phi_{+}(H)=T$. Moreover, the excess of $T$ equals the outer degree of $H$.

Proof. Since the excess $\epsilon$ of $T$ is positive, after doing the closure of $\widehat{T}$ there remains $\epsilon$ cw-outer edge. Thus $H$ is in $\mathcal{J}_{+}$and has outer degree $\epsilon$. Moreover it is clear that, while superimposing $T$ and $H$, we have the local rules indicated in Figure 28 (since these rules are true for the outerplanar map $\widehat{T}$ and are preserved by the closure). Since these rules are also those of Figure 7 (disregarding the incidences with the outer face), we conclude that $T=\Phi_{+}(H)$. Moreover, by Lemma 48, $T$ is a tree, hence a hypermobile. Thus by Lemma 47, $H$ is in $\mathcal{H}_{+}$.

Lemma 50. Let $H \in \mathcal{H}_{+}$, and let $T=\Phi_{+}(H)$. Then $T$ is in $\mathcal{T}_{+}$, and $\Psi_{+}(T)=H$.

Proof. We have proved in Lemma 47 that $T$ is a hypermobile. It remains to show that $\Psi_{+}(T)=H$. First of all, we claim that there exists a "planar matching" of the outer edges of the outerplanar map $\widehat{T}$ of $T$ such that gluing the outer edges of $\widehat{T}$ according to this matching yields $H$. Indeed to obtain the outerplanar map $\widehat{T}$ from $H$, one can apply the following operations illustrated on Figure 29

(i) Replace each 1-way inner edge of $H$ by a pair of parallel 1-way edges, thereby creating a new face of degree 2 .

(ii) For each 1-way edge $e$ with a new face on its right, detach from the origin $v$ of $e$ the sector between $e$ and the next 1-way edge $e^{\prime}$ incident to $v$ in counterclockwise order around $v$; note that $e^{\prime}$ has on its left either a new face or the outer face (see Figure 29).

In order to prove that $\Psi_{+}(T)=H$ it remains to prove that the "planar matching" of the outer edges of $\widehat{T}$ giving $H$ corresponds to the cw-matching of these edges. This is essentially what property $(\boldsymbol{A})$ in Lemma 47 ensures. Indeed, consider a cw-outer edge $e^{\prime}$ and a ccw-outer edge $e^{\prime \prime}$ of $\widehat{T}$ glued into an edge $e$ of $H$, and the sequence $e_{1}, e_{2}, \ldots, e_{n}$ of outer edges of $\widehat{T}$ appearing between $e^{\prime}$ and $e^{\prime \prime}$ in clockwise order around the outer face of $\widehat{T}$. We need to prove that the sequence $e_{1}, e_{2}, \ldots, e_{n}$ is a parenthesis word (when cw-edges are interpreted as $a$ 's and ccw-outer edges are interpreted as $\bar{a}$ 's). By the property 


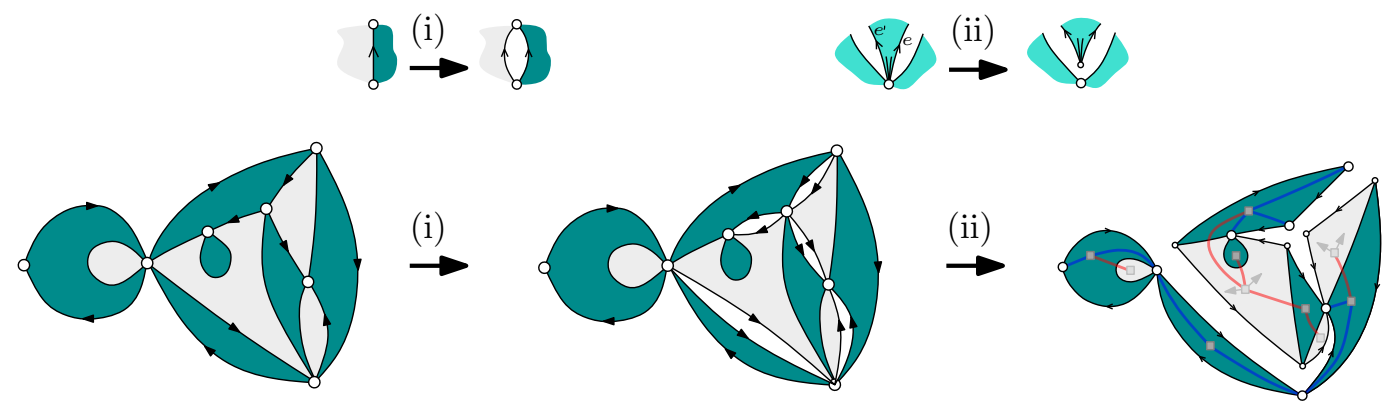

Figure 29. Going from an hyperoriented hypermap $H$ (in $\mathcal{H}_{+}$) to the outerplanar map $\widehat{T}$ of the hypermobile $T=\Phi_{+}(H)$.

applied to $e$, all the outer edges $e_{1}, e_{2}, e_{3}, \ldots, e_{n}$ are glued into edges of $H$ which are inside the cycle $C$ contained in $T \cup\left\{e^{*}\right\}$, hence they are all matched. Moreover, if $e_{i}$ and $e_{j}$ are matched into an edge $\tilde{e}$ of $H$, the property ( $)$ applied to $\tilde{e}$ ensures that $i<j$ (since the cycle $\tilde{C}$ contained in $T \cup\left\{\tilde{e}^{*}\right\}$ lies inside $C$ ). Thus the sequence $e_{1}, e_{2}, \ldots, e_{n}$ is a parenthesis word. Therefore the "planar matching" of the outer edges of $\widehat{T}$ giving $H$ corresponds to the cw-matching of these edges, that is, $H=\Psi_{+}(T)$.

Corollary 49 and Lemma 50 conclude the proof of Theorems 4 and 6 for $\Phi_{+}$.

9.2. Proof for $\Phi_{-}$. The proof for $\Phi_{-}$follows very similar lines. We highlight here the main differences. Let $\mathcal{J}_{-}$be the set of dark-rooted hyperorientations such that the root face contour is simple and every outer edge is ccw-outer, and each incidence of an inner edge $e$ with an outer vertex $v$ is such that $e$ is either 0 -way or 1-way out of $v$. Note that $\mathcal{H}_{-}$ is a subset of $\mathcal{J}_{-}$. We now extend the definition of the mapping $\Phi_{-}$to $\mathcal{J}_{-}$. For $H \in \mathcal{J}_{-}$, we define $\Phi_{-}(H)$ as the map obtained from $H$ by placing a dark (resp. light) square vertex in each dark (resp. light) face, then applying the local rule of Figure 7 to each edge of $H$, and finally deleting the edges of $H$, the dark square vertex $v_{0}$ corresponding to the outer face, the outer vertices of $H$ and the edges between these vertices and $v_{0}$.

Lemma 51. Let $H$ be an hyperoriented hypermap in $\mathcal{J}_{-}$, and let $T=\Phi_{-}(H)$. Then, $T$ is a hypermobile if and only if $H \in \mathcal{H}_{-}$. Moreover, in this case the property (\$) holds for each inner 1-way edge e of $H$.

Proof. The proof is very similar to the proof of Lemma 47. As in Lemma 47, the Euler relation implies that $\Phi_{-}(H)$ is a hypermobile if and only if it is acyclic and the outer face is simple. Next one shows that if $H \notin \mathcal{H}_{-}$then $\Phi_{-}(H)$ has a cycle. The proof is as for $\Phi_{+}$: consider either a counterclockwise cycle or outward cocycle $C$ of $H$, and can prove using the Euler relation that there is a cycle of $T$ in the region of $H$ outside of $C$. The only difference is that when applying the Euler relation, one needs to consider the subgraph $K$ of $T$ made of all its inner vertices outside of $C$ and all its edges outside of $C$. Lastly one shows that if $H \in \mathcal{H}_{-}$then $\Phi_{-}(H)$ is acyclic, and property $(\mathbf{\uparrow})$ holds exactly as for $\Phi_{+}$.

Next we prove that $\Phi_{-}$and $\Psi_{-}$are inverse of each other.

Lemma 52. Let $T$ be a hypermobile of negative excess, and let $H=\Psi_{-}(T)$ be the closure of $T$. Then $T$ is a tree covering all the inner vertices of $H$ (but none of the outer vertices) and all the square vertices placed in the inner faces of $H$.

Proof. The proof of Lemma 52 is the same as the proof of Lemma 48.

Corollary 53. Let $T \in \mathcal{T}_{-}$, and let $H=\Psi_{-}(T)$. Then $H$ is in $\mathcal{H}_{-}$, and $\Phi_{-}(H)=T$. Moreover, the excess of $T$ equals minus the outer degree of $H$. 
Proof. Since the excess $\epsilon$ of $T$ is negative, after doing the closure operations on $T$ there remain $-\epsilon$ ccw-outer edge. Moreover since $T$ covers none of the outer vertices of $H$, each incidence of an inner edge $e$ of $H$ with an outer vertex $v$ is such that $e$ is either 0 -way or 1-way out of $v$. Thus $H$ is in $\mathcal{J}_{-}$and has outer degree $-\epsilon$. Moreover it is clear that superimposing $T$ and $H$ we have the local rules indicated in Figure 28 (since these rules are true for the outerplanar map $\widehat{T}$ and are preserved by the closure), hence $T=\Phi_{-}(H)$. Lastly, by Lemma $48, T$ is a tree, hence a hypermobile. Thus by Lemma $51, H$ is in $\mathcal{H}_{-}$.

Lemma 54. Let $H \in \mathcal{H}_{-}$, and let $T=\Phi_{-}(H)$. Then $T$ is in $\mathcal{T}_{-}$, and $\Psi_{-}(T)=H$.

Proof. The proof of Lemma 54 is the same as the proof of Lemma 50.

Corollary 53 and Lemma 54 conclude the proof of Theorems 4 and 6 for $\Phi_{-}$.

9.3. Proof for $\Phi_{0}$. The proof for $\Phi_{0}$ is again very similar. We define $\mathcal{J}_{0}$ as the family of vertex-rooted hyperorientations such that for each incidence of an edge $e$ with the rootvertex $v_{0}, e$ is either 0 -way or 1 -way out of $v_{0}$. We extend the definition of the mapping $\Phi_{0}$ to $\mathcal{J}_{0}$ : for $H \in \mathcal{J}_{0}$, we define $\Phi_{0}(H)$ as the map obtained from $H$ by placing a dark (resp. light) square vertex in each dark (resp. light) face, then applying the local rule of Figure 7 to each edge of $H$, and finally deleting the edges of $H$, and the root vertex $v_{0}$. In a similar way as for $\Phi_{-}$, one proves:

Lemma 55. Let $H$ be an hyperoriented hypermap in $\mathcal{J}_{0}$, and let $T=\Phi_{0}(H)$. Then, $T$ is a hypermobile if and only if $H \in \mathcal{H}_{0}$. Moreover, in this case the following property holds for each inner 1-way edge e of $H$ :

(\$) Let $u, v$ be the square vertices in the faces incident to e, and let $C$ be the (unique) cycle contained in $T \cup\left\{e^{*}\right\}$, where $e^{*}$ is the edge joining $u$ and $v$ across $e$. Then $e$ is oriented from the region delimited by $C$ containing the root-vertex, to the other region delimited by $C$ (across $\left.e^{*}\right)$.

Then the proof that $\Phi_{0}$ and $\Psi_{0}$ are inverse mappings is similar to the case $\Phi_{-}$. It implies Theorems 4 and 6 for $\Phi_{0}$.

\section{Proofs of Theorems 7, 25, 26, and 27 about CANONICAL ORIENTAtions}

Theorems 25, 26, and 27 state that a hypermap $H$ admits a (unique) $\sigma$-weighted orientation in $\mathcal{H}_{-}, \mathcal{H}_{+}$, or $\mathcal{H}_{0}$ if and only if the charge function $\sigma$ fits $H$. Recall that Theorem 25 actually generalizes Theorem 7 about plane hypermaps (see Lemma 37). In this section, we prove Theorems 25, 26, and 27. The proof is organized as follows.

- In Section 10.1. we prove the necessity of the fitting condition in Theorems 25, 26 and 27.

- In Section 10.2 we develop some tools useful for proving the existence of constrained hyperorientations.

- In Section 10.3 , we prove Theorem 26 in the case where every light face has charge equal to its degree.

- In Section 10.4, we complete the proof of Theorem 26 by reduction to the case treated in Section 10.3

- In Section 10.5. we complete the proof of Theorems 25 and 27 by reduction to Theorem 26

10.1. Necessity of the fitting condition in Theorems 25, 26, and 27, In this subsection we prove the following lemma.

Lemma 56. If a dark-rooted (resp. light-rooted, vertex-rooted) hypermap $H$ admits a $\sigma$ weighted orientation in $\mathcal{H}_{-}$(resp. $\left.\mathcal{H}_{+}, \mathcal{H}_{0}\right)$, then $\sigma$ fits $H$. Moreover if $H$ is dark-rooted, then the contour of the outer face is simple. 
Proof. Let $H$ be a dark-rooted, light-rooted, or vertex-rooted hypermap, and let $\sigma$ be a charge function such that $H$ admits a $\sigma$-weighted hyperorientation $\Omega$ in $\mathcal{H}_{-}, \mathcal{H}_{+}$, or $\mathcal{H}_{0}$. We denote by $w(a)$ the weight of a vertex, edge, or face $a$ of $H$ in $\Omega$.

We first suppose that $H$ is dark-rooted and prove that the contour of the outer face $f_{0}$ of $H$ is simple, the charge of every inner vertex is positive, the charge of every outer vertex is 0 , the charge of the dark outer face $f_{0}$ is $-\operatorname{deg}\left(f_{0}\right)$, and $\sigma_{\text {total }}=0$. By definition of $\mathcal{H}_{-}$the contour of $f_{0}$ is a simple cycle, and since the weight of each outer edge is 1 in $\Omega$, the weight of the outer face is $w\left(f_{0}\right)=\operatorname{deg}\left(f_{0}\right)$. Since, by definition, $w\left(f_{0}\right)=-\sigma\left(f_{0}\right)$, we get $\sigma\left(f_{0}\right)=-\operatorname{deg}\left(f_{0}\right)$. Moreover, by definition, the weight of any outer vertex $v$ is $w(v)=1=\sigma(v)+1$, hence $\sigma(v)=0$. Consider now an inner vertex $v$. Since the orientation $\Omega \in \mathcal{H}_{-}$is accessible from the outer vertices there is a 1-way edge $e$ directed toward $v$, hence $w(v) \geq w(e)>0$. It only remains to prove that $\sigma_{\text {total }}=0$. Let $V, F$, and $K$ be respectively the set of vertices, light faces, and dark faces of $H$. By definition,

$$
\sum_{v \in V} w(v)+\sum_{f \in F} w(f)=\sum_{k \in K} w(k)
$$

and since $\Omega$ is $\sigma$-weighted we get

$$
\left(\sum_{v \in V} \sigma(v)\right)+\operatorname{deg}\left(f_{0}\right)+\left(\sum_{f \in F} \sigma(f)-\operatorname{deg}(f)\right)=\left(\sum_{k \in K}-\sigma(k)-\operatorname{deg}(k)\right)+\operatorname{deg}\left(f_{0}\right) .
$$

Hence, $\sigma_{\text {total }}=\sum_{v \in V} \sigma(v)+\sum_{f \in F} \sigma(f)+\sum_{k \in K} \sigma(k)=0$.

With similar arguments, one proves that if $H$ is light-rooted then the charge of every vertex is positive, the charge of the light outer face $f_{0}$ is $\operatorname{deg}\left(f_{0}\right)$, and $\sigma_{\text {total }}=0$, and if $H$ is vertex-rooted then the charge of every non-root vertex is positive, the charge of the root-vertex is 0 , and $\sigma_{\text {total }}=0$.

It only remains to prove that $H$ satisfies the $\sigma$-girth condition. We first suppose that $H$ is dark-rooted. Let $R$ be a light region. Let $V, E, F$ and $K$ be respectively the set of vertices strictly inside $R$, edges strictly inside $R$, light faces inside $R$, and dark faces inside $R$. We want to prove

$$
|\partial R| \geq \sigma(R):=\sum_{v \in V} \sigma(v)+\sum_{f \in F} \sigma(f)+\sum_{k \in K} \sigma(k),
$$

with strict inequality if every outer vertex is strictly in $R$.

Because $\Omega$ is $\sigma$-weighted we get

$$
\begin{aligned}
\sum_{v \in V} \sigma(v) & =-b+\sum_{v \in V} w(v) \\
\sum_{f \in F} \sigma(f) & =|E|+|\partial R|+\sum_{f \in F} w(f), \\
\sum_{k \in K} \sigma(k) & =-|E|+\mathbf{1}_{f_{0} \in R} \cdot \operatorname{deg}\left(f_{0}\right)-\sum_{k \in K} w(k),
\end{aligned}
$$

where $b$ is the number of outer vertices in $V$, and $f_{0}$ is the dark outer face. Hence

$$
\sigma(R)=|\partial R|-b+\mathbf{1}_{f_{0} \in R} \cdot \operatorname{deg}\left(f_{0}\right)+\sum_{v \in V} w(v)+\sum_{f \in F} w(f)-\sum_{k \in K} w(k),
$$

and the requirement (5) becomes

$$
\sum_{k \in K} w(k)-\sum_{v \in V} w(v)-\sum_{f \in F} w(f) \geq \mathbf{1}_{f_{0} \in R} \cdot \operatorname{deg}\left(f_{0}\right)-b,
$$

Moreover we have

$$
\sum_{k \in K} w(k)-\sum_{v \in V} w(v)-\sum_{f \in F} w(f)=x-y \geq x
$$


where $x$ is the sum of the (positive) weights of the 1-way edges in $E$ oriented toward vertices incident to edges in $\partial R$, and $y$ is the sum of the (non-positive) weights of the 0 -way edges in $\partial R$. If $f_{0} \notin R$, then $b=0$ and the inequality (6) holds because $x \geq 0$. If $f_{0} \in R$ and $b=\operatorname{deg}\left(f_{0}\right)$ (i.e. every outer vertex is strictly inside $R$ ), then inequality $(6)$ is strict because $x>0$ (indeed, since $\Omega$ is accessible from the outer vertices of $H$, there exists a 1-way edge in $E$ oriented toward vertices of $\partial R)$. Lastly suppose that $f_{0} \in R$ and $b<\operatorname{deg}\left(f_{0}\right)$. Because $f_{0}$ is a dark face, all the edges incident to $f_{0}$ are in $E$, and because $\Omega \in \mathcal{H}_{-}$these edges are 1-way and have weight 1 . Thus for each outer vertex $v$ on $\partial R$ there is an edge in $E$ of weight 1 oriented toward $v$. Hence $x \geq \operatorname{deg}\left(f_{0}\right)-b$ which is the number of outer vertices on $\partial R$. This proves (6) and completes that proof that $H$ satisfies the $\sigma$-girth condition when $H$ is dark-rooted.

The case where $H$ is light-rooted (resp. vertex-rooted) is similar. Indeed, by the same arguments, we see that the $\sigma$-girth condition a light region $R$ becomes the following requirement:

$$
\sum_{k \in K} w(k)-\sum_{v \in V} w(v)-\sum_{f \in F} w(f) \geq 0,
$$

with strict inequality if one of the outer edges is strictly inside $R$ (resp. if the root vertex is strictly inside $R$ ). This is easily seen to hold with arguments similar to the ones above. The only point that requires a special argument is that the equality is strict if $H$ is light-rooted and one of the outer edges is strictly inside $R$. For this particular case, we need to prove that the sum $x$ of weights of the 1-way edges in $E$ oriented toward vertices incident to edges of $\partial R$ is positive. This holds, because if one of the outer vertices $v$ is strictly inside $R$ then $x>0$ because the vertices on $\partial R$ are accessible from $v$, while if none of the outer vertices is strictly in $R$, then the outer edge $e$ strictly inside $R$ is a 1-way edge in $E$ oriented toward a vertex of $\partial R$ (indeed, $e$ is 1 -way because $\Omega \in \mathcal{H}_{+}$).

10.2. A preliminary result about $\alpha$-hyperflows. In this subsection we prove a result akin to the mincut-maxflow theorem for the hyperflows of bipartite graphs. This result will then be used in Section 10.3

Throughout this subsection, we fix a (finite, undirected) bipartite graph $G=(X \sqcup Y, E)$ where every edge $e \in E$ joins a vertex in $X$ to a vertex in $Y$. We call hyperflow of $G$, a function $\varphi$ from the edge set $E$ to the set $\mathbb{R}^{+}$of non-negative real numbers. Let $P$ be a directed path, or cycle, of $G$ and let $P_{X}$ be the subset of edges of $P$ oriented toward a vertex in $X$. Given a hyperflow $\varphi$ of $G$, we say that $P$ is $\varphi$-positive if $\varphi(e)>0$ for every edge $e \in P_{X}$. A $\varphi$-positive path is represented in Figure 30 (a). For a vertex $x_{0} \in X$, we say that a hyperflow $\varphi$ is accessible from $x_{0}$ if for all $x \in X$ there is a $\varphi$-positive path from $x_{0}$ to $x$. For instance, The hyperflow represented in Figure 30 (a) is accessible from $x_{0}$.

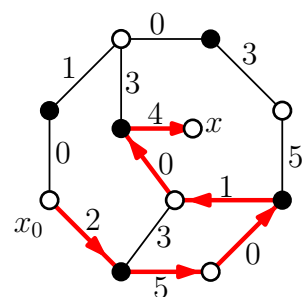

(a)

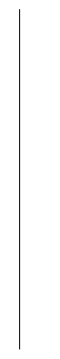

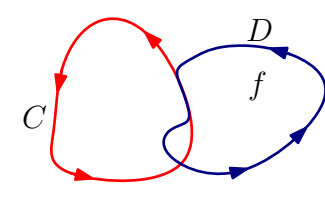

(b)

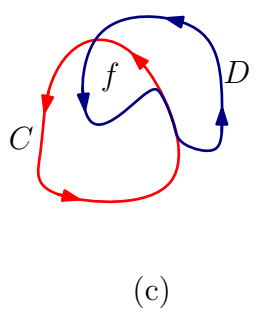

(c)

Figure 30. (a): A bipartite graph endowed with a hyperflow $\varphi$, and a $\varphi$-positive directed path from $x_{0}$ to $x$. The vertices in $X$ and $Y$ are represented in light and dark respectively and the value of $\varphi$ is indicated on each edge. (b),(c): The cycles $C$ and $D$ in the proof of Lemma 59 . 
Let $\varphi$ be a hyperflow of $G=(X \sqcup Y, E)$. We call $\varphi$-flow at a vertex $v \in X \sqcup Y$ the sum

$$
\phi(v):=\sum_{e \in E \text { incident to } v} \varphi(e) .
$$

Given a function $\alpha$ from $X \sqcup Y$ to $\mathbb{R}^{+}$, we say that $\varphi$ is an $\alpha$-hyperflow if the $\varphi$-flow at every vertex $v \in X \sqcup Y$ is equal to $\alpha(v)$. We now establish a criterion for the existence of an accessible $\alpha$-hyperflow:

Lemma 57. Let $\alpha$ be a function from $X \sqcup Y$ to $\mathbb{R}^{+}$. For a subset $A \subseteq X$, let us denote

$$
\alpha(A):=\sum_{x \in X} \alpha(x)-\sum_{y \in Y_{A}} \alpha(y),
$$

where $Y_{A}$ denotes the set of vertices in $Y$ having all their neighbors in $A$. Then there exists an $\alpha$-hyperflow of $G$ if and only if

$$
\forall A \subseteq X, \alpha(A) \geq 0,
$$

with equality for $A=X$. Moreover for any vertex $x_{0} \in X$ and any $\alpha$-hyperflow $\varphi$, the hyperflow $\varphi$ is accessible from $x_{0}$ if and only if $\alpha(A)>0$ for all non-empty subset $A \subset X$ not containing $x_{0}$.

Proof. First suppose that there exists an $\alpha$-hyperflow $\varphi$ of $G$. In this case, for all $A \subseteq X$,

$$
\sum_{x \in A} \alpha(x)=\sum_{e \text { incident to } A} \varphi(e) \geq \sum_{y \in Y_{A}} \alpha(y),
$$

with equality if $A=X$. Hence $\alpha(A) \geq 0$, with equality for $A=X$.

We will now prove that an $\alpha$-hyperflow exists whenever $\alpha(A) \geq 0$ for all $A \subseteq X$, with equality for $A=X$. We make an induction on $|X \cup Y \cup E|$. The property is trivial when $E=\emptyset$, hence for the induction step we can assume $E \neq \emptyset$. We consider an edge $e_{0} \in E$ with endpoints $x_{0} \in X$ and $y_{0} \in Y$. For $\epsilon \geq 0$ we denote by $\alpha_{\epsilon}$ the function from $X \sqcup Y$ to $\mathbb{R}^{+}$defined by: $\alpha_{\epsilon}\left(x_{0}\right)=\alpha\left(x_{0}\right)-\epsilon, \alpha_{\epsilon}\left(y_{0}\right)=\alpha\left(y_{0}\right)-\epsilon$ and $\alpha_{\epsilon}(z)=\alpha(z)$ for all $z \neq x_{0}, y_{0}$. Observe that if $\varphi$ is an $\alpha_{\epsilon}$-hyperflow of $G$, then $\varphi^{\prime}$ defined by $\varphi^{\prime}\left(e_{0}\right)=\varphi\left(e_{0}\right)+\epsilon$ and $\varphi^{\prime}(e)=\varphi(e)$ for all $e \neq e_{0}$ is an $\alpha$-hyperflow of $G$. Hence it suffices to prove that there exists an $\alpha_{\epsilon}$-hyperflow of $G$ for some $\epsilon \geq 0$. We choose $\epsilon$ maximal such that $\alpha_{\epsilon}\left(x_{0}\right) \geq 0$, $\alpha_{\epsilon}\left(y_{0}\right) \geq 0$, and $\alpha_{\epsilon}(A) \geq 0$ for all $A \subseteq X$. Clearly, $\alpha_{\epsilon}(X)=\alpha(X)=0$, and $\alpha_{\epsilon}(A) \geq 0$ for all $A \subseteq X$. Moreover, we have either $\alpha_{\epsilon}\left(x_{0}\right)=0$, or $\alpha_{\epsilon}\left(y_{0}\right)=0$ or $\alpha_{\epsilon}(A)=0$ for some $A \neq \emptyset, X$. Suppose first $\alpha_{\epsilon}\left(x_{0}\right)=0$. In this case we consider the subgraph $G^{\prime}$ obtained from $G$ by deleting $x_{0}$ and the incident edges, and we denote by $\alpha^{\prime}$ the restriction of $\alpha_{\epsilon}$ to $G^{\prime}$. Clearly $\alpha^{\prime}(A) \geq 0$ for all $A \subseteq X \backslash\left\{x_{0}\right\}$, with equality for $A=X \backslash\left\{x_{0}\right\}$. Hence by the induction hypothesis, there exists an $\alpha^{\prime}$-hyperflow of $G^{\prime}$ and this gives an $\alpha_{\epsilon}$-hyperflow of $G$ (by setting the flow on edges incident to $x_{0}$ to be 0 ), and hence an $\alpha$-hyperflow of $G$. The case $\alpha_{\epsilon}\left(y_{0}\right)=0$ is similar. Suppose lastly that $\alpha_{\epsilon}(A)=0$ for some subset $A \neq \emptyset, X$. Let $\bar{A}=X \backslash A$ and $\overline{Y_{A}}=Y \backslash Y_{A}$. Let $G_{1}$ (resp. $G_{2}$ ) be the graph with vertex set $A \cup Y_{A}$ (resp. $\bar{A} \cup \overline{Y_{A}}$ ) and edge set $E_{1}$ (resp. $E_{2}$ ) made of all the edges with both endpoints in $A \cup Y_{A}$ (resp. $\bar{A} \cup \overline{Y_{A}}$ ). Observe that the graph $G_{1} \cup G_{2}$ is simply obtained from $G$ by deleting the set $E_{0}$ of edges having both endpoints in $A \cup \overline{Y_{A}}$; see Figure 31 . We denote by $\alpha^{\prime}$ and $\alpha^{\prime \prime}$ respectively the restriction of $\alpha_{\epsilon}$ to $G_{1}$ and $G_{2}$. Observe that for all $B \subseteq A$, the set of vertices of $G_{1}$ with all their neighbors in $B$ is $Y_{B \cup \bar{A}} \cap Y_{A}=Y_{B}$. Thus

$$
\alpha^{\prime}(B)=\sum_{x \in B} \alpha_{\epsilon}(x)-\sum_{y \in Y_{B}} \alpha_{\epsilon}(y)=\alpha_{\epsilon}(B) .
$$

Hence $\alpha^{\prime}(B) \geq 0$ for all $B \subseteq A$, with equality for $B=A$. Hence, by the induction hypothesis, there exists a $\alpha^{\prime}$-hyperflow $\varphi_{1}$ of $G_{1}$. Now for $B \subseteq \bar{A}$, the set of vertices of $G_{2}$ 
with all their neighbors in $B$ is $Y_{A \cup B} \cap \overline{Y_{A}}$. Hence

$$
\begin{aligned}
\alpha^{\prime \prime}(B) & =\sum_{x \in B} \alpha_{\epsilon}(x)-\sum_{Y_{B \cup A} \cap \overline{Y_{A}}} \alpha_{\epsilon}(y) \\
& =\left(\sum_{x \in A \cup B} \alpha_{\epsilon}(x)-\sum_{x \in A} \alpha_{\epsilon}(x)\right)-\left(\sum_{y \in Y_{A \cup B}} \alpha_{\epsilon}(y)-\sum_{y \in Y_{A}} \alpha_{\epsilon}(y)\right)=\alpha_{\epsilon}(A \cup B) .
\end{aligned}
$$

Hence $\alpha^{\prime \prime}(B) \geq 0$ for all $B \subseteq \bar{A}$, with equality for $B=\bar{A}$. Hence, by the induction hypothesis, there exists an $\alpha^{\prime \prime}$-hyperflow $\varphi_{2}$ of $G_{2}$. We now consider the hyperflow $\varphi$ of $G$ defined by $\varphi(e)=0$ if $e \in E_{0}, \varphi(e)=\varphi_{1}(E)$ if $E$ in $E_{1}$, and $\varphi(e)=\varphi_{2}(e)$ if $e \in E_{2}$. It is clear that $\varphi$ is an $\alpha_{\epsilon}$-hyperflow. This completes the proof by induction.

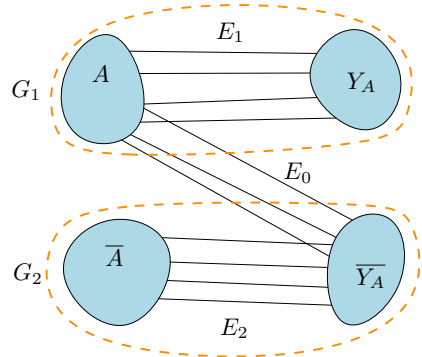

FIgURE 31. The bipartite graph $G=(X \sqcup Y, E)$, and the subgraphs $G_{1}$ and $G_{2}$. We have $X=A \cup \bar{A}, Y=Y_{A} \cup \overline{Y_{A}}$ and $E=E_{0} \cup E_{1} \cup E_{2}$.

It remains to prove that an $\alpha$-hyperflow $\varphi$ is accessible from a vertex $x_{0} \in X$ if and only if $\alpha(A)>0$ for all non-empty subset $A \subset X$ not containing $x_{0}$. Suppose first that $\varphi$ is accessible from $x_{0}$ and let $A \subset X$ be a non-empty subset not containing $x_{0}$. Let $P$ be a $\varphi$-positive path from $x_{0}$ to a vertex $x \in A$. Let $e_{0}$ be the first edge of $P$ incident to a vertex in $A$. This edge of $P$ is directed from its endpoint $y \in Y$ to its endpoint $a \in A$, hence $\varphi\left(e_{0}\right)>0$. Moreover $y \notin Y_{A}$, hence

$$
\sum_{x \in A} \alpha(x)=\sum_{e \text { incident to } A} \varphi(e) \geq \varphi\left(e_{0}\right)+\sum_{y \in Y_{A}} \alpha(y)
$$

Thus $\alpha(A) \geq \varphi\left(e_{0}\right)>0$, as wanted. Suppose now that $\varphi$ is not accessible from $x_{0}$. Consider the set $A$ of vertices $x \in X$ such that there exists no $\varphi$-positive path from $x_{0}$ to $x$. This definition implies that every edge $e$ incident to a vertex $x \in A$ and a vertex $y \in Y \backslash Y_{A}$ satisfies $\varphi(e)=0$. Thus

$$
\sum_{x \in A} \alpha(x)=\sum_{e \text { incident to } A} \varphi(e)=\sum_{e \text { incident to } Y_{A}} \varphi(e)=\sum_{y \in Y_{A}} \alpha(y) .
$$

Hence $\alpha(A)=0$ for a non-empty set $A \subset X$ not containing $x_{0}$.

Remark 58. In the literature, $\alpha$-hyperflows are also known as b-matchings [37, Chap. 21]. Our existence criterion in Lemma 57 can be checked to be equivalent to Corollary 21.1b from 37. (we have provided our own proof and terminology for completeness and convenience). About efficiently computing an $\alpha$-hyperflow of $G=(V, E)$, when $\alpha$ only has integer values the problem can easily be reduced to that of finding a perfect matching in a bipartite graph $G^{\prime}=\left(V^{\prime}, E^{\prime}\right)$ associated to $G$ (each vertex $v \in G$ is turned into $\alpha(v)$ copies in $G^{\prime}$, and for each edge $(u, v) \in G$, there is an edge in $G^{\prime}$ between every copy of $u$ and every copy of $v$ ). The algorithm of Hopcroft and Karp [23] yields a perfect matching of $G^{\prime}$ in time $O\left(\sqrt{\left|V^{\prime}\right|}\left|E^{\prime}\right|\right)$, which is $O(c \sqrt{|V|}|E|)$, with $c=\left(\sum_{v \in V} \alpha(v)\right)^{1 / 2} \sum_{(u, v) \in E} \alpha(u) \alpha(v)$. A detailed survey on complexity results (for the general case of flow values in $\mathbb{R}^{+}$) is given in [37, Chap. 21]. 
Suppose that a bipartite graph $G=(X \sqcup Y, E)$ is embedded (i.e., drawn without edge crossings) in the plane. In this case, a directed cycle $C$ of $G$ is called counterclockwise if the outer face of $G$ lies to the right of $C$. A hyperflow $\varphi$ of $G$ is called minimal if there is no $\varphi$ positive counterclockwise directed cycle of $G$. The hyperflow $\varphi$ represented in Figure 30(a) is not minimal because there is a $\varphi$-positive counterclockwise directed cycle of length 4 .

Lemma 59. Let $G=(X \sqcup Y, E)$ be a bipartite graph embedded in the plane. If $\alpha$ is a function from $X \sqcup Y$ to $\mathbb{R}^{+}$such that there exists an $\alpha$-hyperflow of $G$, then there exists a unique minimal $\alpha$-hyperflow of $G$.

Proof. We first prove the existence of a minimal $\alpha$-hyperflow. We first define the operation of pushing a cycle. Let $\varphi$ be an $\alpha$-hyperflow, and let $C$ be a $\varphi$-positive counterclockwise directed cycle. Let $C_{X}$ (resp. $C_{Y}$ ) be the subset of edges of the directed cycle $C$ oriented toward a vertex in $X$ (resp. $Y$ ). Let $m=\min \left\{\varphi(e), e \in C_{X}\right\}$ and let $\psi$ be the hyperflow defined by $\psi(e)=\varphi(e)-m$ if $e \in C_{X}, \psi(e)=\varphi(e)+m$ if $e \in C_{Y}$, and $\psi(e)=\varphi(e)$ if $e$ is not in $C$. Observe that $\psi$ is an $\alpha$-hyperflow. We say that $\psi$ is the $\alpha$-hyperflow obtained from $\varphi$ by pushing the cycle $C$. We will now prove that the minimal $\alpha$-hyperflow can be obtained from any $\alpha$-hyperflow by repeatedly pushing counterclockwise directed cycles. For an $\alpha$ hyperflow $\varphi$ we consider the total number $N(\varphi)$ of faces which are enclosed in (i.e., separated from the outer face by) a $\varphi$-positive counterclockwise directed cycle. By definition, an $\alpha$ hyperflow $\varphi$ is minimal if and only if $N(\varphi)=0$. Hence it is sufficient to show that for any non-minimal $\alpha$-hyperflow $\varphi$ there is an $\alpha$-hyperflow $\psi$ obtained from $\varphi$ by pushing a $\varphi$-positive counterclockwise directed cycle such that $N(\psi)<N(\varphi)$. Let $\varphi$ be a non minimal $\alpha$-hyperflow, and let $C$ be a $\varphi$-positive counterclockwise directed cycle $C$ enclosing a maximal number of faces. We consider the $\alpha$-hyperflow $\psi$ obtained from $\varphi$ by pushing the cycle $C$. Now consider a face $f$ not enclosed by a $\varphi$-positive counterclockwise directed cycle. If $f$ is enclosed by a $\psi$-positive counterclockwise directed cycle $D$, then $D$ must have an edge in $C_{Y}$. But this would imply the existence of a $\varphi$-positive counterclockwise directed cycle $D^{\prime} \subset C \cup D$ enclosing $f$ and all the faces inside $C$ : see Figure 30(b). This is impossible by the choice of the cycle $C$. Consider now a face $f$ inside $C$ and incident to an edge of $C$. This face cannot be inside a $\psi$-positive counterclockwise directed cycle $D$, otherwise $D$ would cross $C$, and there would be again a $\varphi$-positive counterclockwise directed cycle $D^{\prime} \subset C \cup D$ enclosing more faces than $C$ : see Figure 30(c). This is impossible by the choice of the cycle $C$. Thus $N(\psi)<N(\varphi)$ as wanted. This proves the existence to a minimal $\alpha$-hyperflow.

We now prove the uniqueness of the minimal $\alpha$-hyperflow. Suppose that $\varphi$ and $\psi$ are distinct $\alpha$-hyperflows. We want to show that they are not both minimal. Let $e_{1}$ be an edge such that $\varphi\left(e_{1}\right)<\psi\left(e_{1}\right)$. Let $x_{1} \in X$ and $y_{1} \in Y$ be the endpoints of $e$. Since

$$
\sum_{e \text { incident to } y_{1}} \varphi(e)=\alpha\left(y_{1}\right)=\sum_{e \text { incident to } y_{1}} \psi(e),
$$

there exists an edge $e_{1}^{\prime} \neq e_{1}$ incident to $y_{1}$ such that $\varphi\left(e_{1}^{\prime}\right)>\psi\left(e_{1}^{\prime}\right)$. Continuing in this way, one find a directed path made of edges $e_{1}, e_{1}^{\prime}, e_{2}, e_{2}^{\prime}, e_{3}, e_{3}^{\prime}, \ldots$ such that $\varphi\left(e_{i}\right)<\psi\left(e_{i}\right)$ and $\varphi\left(e_{i}^{\prime}\right)>\psi\left(e_{i}^{\prime}\right)$. This path will eventually intersect itself, so we get a directed simple cycle $C$ of $G$ such that $C$ is $\psi$-positive and the directed cycle $C^{\prime}$ obtained by reversing $C$ is $\varphi$-positive. Either $C$ or $C^{\prime}$ is counterclockwise, hence $\varphi$ and $\psi$ are not both minimal.

Remark 60 . When $\alpha$ has only integer values and $G$ has at least one $\alpha$-hyperflow, more can be said on the structure of the set $K$ of $\alpha$-hyperflows of $G$ such that all flow-values are integers. By a result of Felsner and Knauer [20, Sec.4.2] (extending an earlier result by Khuller et al. 24]), the set $K$ carries the structure of a distributive lattice (their result is formulated on flows of directed graphs with prescribed flow-excess at each vertex, which are equivalent to our formulation of $\alpha$-hyperflows upon orienting all the edges from black to white vertices); and naturally the minimum element in the lattice is the minimal $\alpha$ hyperflow. This is an extension of a well-known result of Propp [34] and Felsner [19] on 
$\alpha$-orientations of planar maps (an $\alpha$-orientation is an orientation where every vertex $v$ has outdegree $\alpha(v))$ : Propp and Felsner have shown that, if non-empty, the set of $\alpha$-orientations of a map embedded in the plane is a distributive lattice, the minimum element of which is the unique $\alpha$-orientation with no clockwise cycle.

About algorithmic aspects, it should be doable to compute the minimal $\alpha$-hyperflow in linear time once an $\alpha$-hyperflow is computed (which has superlinear complexity as we have seen in Remark 58, by extending the approach described in 14 for $\alpha$-orientations.

Lastly we prove an additional technical lemma about the minimal hyperflow.

Lemma 61. Let $G=(X \sqcup Y, E)$ and $\alpha$ be as in Lemma 59, and let $\varphi_{0}$ be the minimal $\alpha$-hyperflow of $G$. Let $x \in X, y \in Y$, and let $a=(x, y)$ be an edge of $G$ such that the face on the right of a (when oriented from $x$ to $y$ ) is the outer face. If there is an $\alpha$-hyperflow $\varphi$ such that $\varphi(a)>0$, then $\varphi_{0}(a)>0$.

Proof. Let $\varphi$ be a $\alpha$-hyperflow such that $\varphi(a)>0$. It was shown in the proof of Lemma 59 that the minimal $\alpha$-hyperflow $\varphi_{0}$ can be obtained from $\varphi$ by repeatedly pushing counterclockwise directed cycles. Moreover, because the face on the right of $a$ is the outer face, for any counterclockwise directed cycle $C$, the edge $a$ belongs to the subset $C_{Y}$ of edges of $C$ oriented toward a vertex in $Y$. Thus pushing cycles will only increase the value of the hyperflow on $a$, so $\varphi_{0}(a) \geq \varphi(a)>0$.

10.3. Proof of Theorem 26 when the charge of every light face is equal to its degree. This subsection is devoted to the proof of the following result.

Proposition 62. Let $H$ be a light-rooted hypermap. Let $\sigma$ be a charge function which fits $H$ and such that every light face has charge equal to its degree. Then $H$ admits a unique $\sigma$-weighted hyperorientation in $\mathcal{H}_{+}$.

Throughout this subsection $(H, \sigma)$ is a charged hypermap satisfying the hypotheses of Proposition 62. We say that a weighted hyperorientation of $H$ is $\mathbb{R}^{+}$-weighted if 0 -way edges have weight 0 , and 1 -way edges have positive real weights. In fact, the $\sigma$-weighted hyperorientations of $H$ are precisely the $\mathbb{R}^{+}$-weighted hyperorientations such that

- every vertex has weight $\sigma(v)$,

- every inner dark face $f$ has weight $-\sigma(f)-\operatorname{deg}(f)$.

We will prove Proposition 62 in two steps. First we will establish the existence of a certain $\alpha$-hyperflow in a related graph $G_{H}$ using Lemma 57, and then we will use this $\alpha$-hyperflow to define a $\sigma$-weighted hyperorientation of $H$.

We call star graph of $H$ the bipartite graph $G_{H}$ (embedded in the plane) obtained as follows: for each dark face $h$ of $H$, place a vertex $y$ of $G_{H}$ inside $h$ and draw an edge $e$ of $G_{H}$ going from $y$ to each corner of $h$. The construction is illustrated in Figure 32 . We denote by $X$ the vertex set of $H$, and by $Y$ the remaining set of vertices of $G_{H}$ (which are placed inside the dark faces of $H$ ).
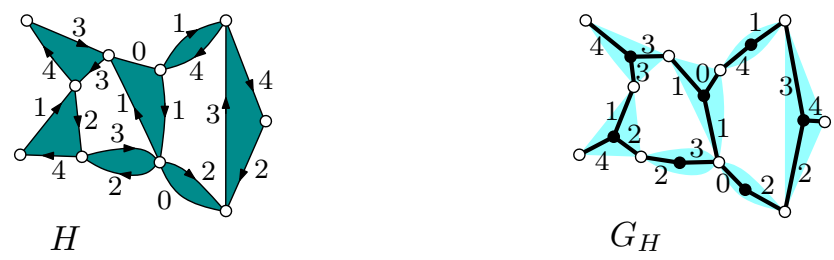

Figure 32. A hypermap $H$ and the associated star graph $G_{H}$. The bipartite map $G_{H}$ is endowed with a hyperflow $\varphi$, while $H$ is endowed with the $\mathbb{R}^{+}$-weighted hyperorientation $\Gamma(\varphi)$.

Given a hyperflow $\varphi$ of $G_{H}$, we define an $\mathbb{R}^{+}$-weighted hyperorientations $\Gamma(\varphi)$ of $H$ as follows: 
- for every edge $e$ of $G_{H}$, we give weight $\varphi(e)$ to the edge $e^{\prime}$ of $H$ preceding $e$ clockwise around the endpoint of $e$ in $X$

- we orient $e^{\prime} 1$-way if $\varphi(e)>0$ and 0 -way otherwise.

The mapping $\Gamma$ is illustrated in Figure 32. It is clear that $\Gamma$ is a bijection between the hyperflows of $G_{H}$ and the $\mathbb{R}^{+}$-weighted hyperorientations of $H$. Moreover, the $\varphi$-flow at a vertex $v$ of $G_{H}$ is equal to the weight of the corresponding vertex or dark face of $H$ in the hyperorientation $\Gamma(\varphi)$. This proves the following result.

Lemma 63. The mapping $\Gamma$ is a bijection between the $\sigma$-weighted hyperorientations of $H$ and the $\alpha$-hyperflows of $G_{H}$, where $\alpha$ is the function defined on $X \sqcup Y$ by

- for every vertex $x \in X, \alpha(x)=\sigma(x)$,

- for every vertex $y \in Y, \alpha(y)=-\sigma\left(f_{y}\right)-\operatorname{deg}\left(f_{y}\right)$, where $f_{y}$ is the dark face of $H$ containing $y$.

We will now prove the existence of a minimal $\alpha$-hyperflow for $G_{H}$ by using Lemmas 57 and 59

Lemma 64. Let $\alpha$ be the function defined in Lemma63. Then $G_{H}$ admits a unique minimal $\alpha$-hyperflow $\varphi$. Moreover this hyperflow is accessible from every outer vertex of $H$.

Proof. For $A \subseteq X$, we denote by $Y_{A}$ the set of vertices of $G_{H}$ placed in the inner dark faces of $H$ having all of their incident vertices in $A$ and we let

$$
\alpha(A):=\sum_{x \in A} \alpha(x)-\sum_{y \in Y_{A}} \alpha(y) .
$$

By Lemmas 57 and 59 , the existence and uniqueness of $\varphi$ are granted provided $\alpha(A) \geq 0$ for all $A \subseteq X$ with equality for $A=X$. We denote by $G_{A}=\left(A \cup Y_{A}, E_{A}\right)$ the subgraph of $G_{H}$ induced by $A \cup Y_{A}$ (that is, $E_{A}$ is the set of edges of $G_{H}$ with both endpoints in $\left.A \cup Y_{A}\right)$. See for instance Figure 33(a). Since $\alpha(X)$ is linear over the connected components of the subgraph $G_{A}$, it is sufficient to prove $\alpha(A) \geq 0$ when $G_{A}$ is connected (with equality for $A=X)$.

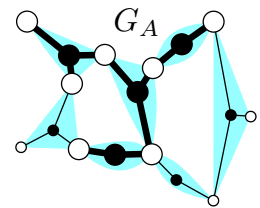

(a)

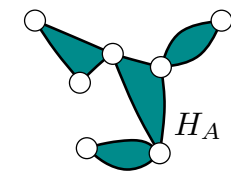

(b)

Figure 33. (a) A subgraph $G_{A}=\left(A \cup Y_{A}, E_{A}\right)$ of the star graph $G_{H}$ : the vertices in $A \cup Y_{A}$ are represented by big discs and the edges in $E_{A}$ are represented in bold lines. (b) The hypermap $H_{A}$.

Let $A \subseteq X$ be such that $A \neq \emptyset$ and $G_{A}$ is connected. Observe that $G_{A}$ is the star graph of a hypermap $H_{A}$ with a light outer face: the set of vertices of $H_{A}$ is $A$ and the set of dark faces of $H_{A}$ is the set of inner dark faces of $H$ having all their incident vertices in $A$. See for instance Figure 33(b). Let $D_{A}$ and $L_{A}$ be the set of dark and light faces of $H_{A}$. By definition of $\alpha$, we get

$$
\alpha(A)=\sum_{x \in A} \sigma(x)+\sum_{f \in D_{A}}(\sigma(f)+\operatorname{deg}(f))=\sum_{x \in A} \sigma(x)+\sum_{f \in D_{A}} \sigma(f)+\sum_{\ell \in L_{A}} \operatorname{deg}(\ell) .
$$

Now every face $\ell \in L_{A}$ corresponds to a light region of $H$, thus the $\sigma$-girth condition gives

$$
\operatorname{deg}(\ell) \geq \sum_{x \text { vertex of } H \text { strictly inside } \ell} \sigma(x)+\sum_{f \text { face of } H \text { inside } \ell} \sigma(f),
$$


with strict inequality for the outer face $\ell_{0}$ of $H_{A}$ if $\ell_{0}$ is not equal to the outer face of $H$. Thus,

$$
\sum_{\ell \in L_{A}} \operatorname{deg}(\ell) \geq \sum_{x \text { vertex of } H \text { not in } A} \sigma(x)+\sum_{f \text { face of } H \text { not in } D_{A}} \sigma(f),
$$

with strict inequality if the outer face of $H_{A}$ is not equal to the outer face of $H$. This gives

$$
\alpha(A) \geq \sigma_{\text {total }}=0 .
$$

Moreover, if one of the outer vertices is not in $A$, then one of the dark faces incident to the outer edges is not in $D_{A}$, hence the inequality is strict: $\alpha(A)>\sigma_{\text {total }}=0$. Thus, by Lemmas 57 and 59 , the graph $G_{H}$ admits a unique minimal $\alpha$-hyperflow $\varphi$, and $\varphi$ is accessible from every outer vertex.

Next we use lemma 64 to prove the following.

Lemma 65. The hypermap $H$ admits a unique $\sigma$-weighted hyperorientation $\Omega$ which is both minimal and accessible from the outer vertices.

Proof. Existence. By Lemma 64, the bipartite graph $G_{H}$ admits a unique minimal $\alpha$ hyperflow $\varphi$. By Lemma 63 , we know that $\Omega=\Gamma(\varphi)$ is a $\sigma$-weighted hyperorientation of $H$. We now want to prove that $\Omega$ is both minimal and accessible from the outer vertices.

We first prove that $\Omega$ is minimal. Suppose, by contradiction, that there is a simple counterclockwise directed cycle $C$ of $\Omega$ distinct from the outer face. We will show that in this case, there is a $\varphi$-positive counterclockwise directed cycle $D$ of $G_{H}$; see Figure 34 . Let $e_{1}, \ldots, e_{k}$ be the oriented edges of $C$. Let $f_{i}$ be the dark face of $H$ incident to $e_{i}$ (which is on the right of $e_{i}$ ). Let $x_{i}, x_{i}^{\prime} \in X$ be the origin and end of $e_{i}$, and let $y_{i} \in Y^{\prime}$ be the vertex of $G_{H}$ in the dark face $f_{i}$. Let $a_{i}, a_{i}^{\prime}$ be the oriented edges $\left(x_{i}, y_{i}\right)$ and $\left(y_{i}, x_{i}^{\prime}\right)$; see Figure 34 The edges $a_{1}, a_{1}^{\prime}, \ldots, a_{k}, a_{k}^{\prime}$ form a counterclockwise directed cycle $D$ of $G_{H}^{\prime}=\left(X \sqcup Y^{\prime}, E^{\prime}\right)$, hence it contains a simple counterclockwise directed cycle $D^{\prime}$. By definition, the hyperflow $\varphi^{\prime}\left(a_{i}^{\prime}\right)$ is equal to the weight of $e_{i}$ which is positive. Hence the counterclockwise directed cycle $D^{\prime}$ is $\varphi$-positive. This is a contraction since the hyperflow $\varphi$ is minimal.

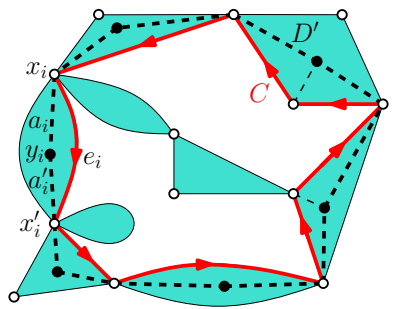

Figure 34. A counterclockwise directed cycle $C$ of $H$ (thick lines) and the corresponding $\varphi$-positive counterclockwise directed simple cycle $D^{\prime}$ of $G_{H}$ (thick dashed lines).

We now want to prove that $\Omega$ is accessible from every outer vertex of $H$. Let $u_{0}$ be an outer vertex of $H$, and let $v$ be an inner vertex. We want to exhibit a directed path of $H$ from $u_{0}$ to $v$. We know (by Lemma 64) that the hyperflow $\varphi$ of $G_{H}$ is accessible from $u_{0}$, hence for every vertex $u$ of $H$ there exists a $\varphi$-positive path of $G_{H}$ from $u_{0}$ to $u$. For a vertex $u \neq u_{0}$ of $H$, we consider the set $A_{u}$ of all the edges of $G_{H}$ incident to $u$ which are part of a $\varphi$-positive simple directed path of $G_{H}$ from $u_{0}$ to $u$. For a 1-way edge $e$ of $H$ having origin $u \neq u_{0}$, we denote by $\theta(e)$ the edge of $A_{u}$ preceding $e$ in clockwise direction around $u$, and we denote by $\pi(e)$ the edge of $H$ following $\theta(e)$ around $u$; see Figure 35 (a). By definition, $\varphi(\theta(e))>0$ hence $\pi(e)$ is a 1-way edge of $H$ directed toward $u$ in $\Omega$. Moreover, there exists no $\varphi$-positive (simple) directed path of $G_{H}$ from $u_{0}$ to $u$ ending between $e$ and $\pi(e)$ in clockwise direction around $u$. We now construct a directed path of $H$ ending at $v$ 
as follows. First we choose an edge $a \in A_{v}$ and denote by $e_{0}$ the edge of $H$ following $a$ clockwise around $v$. The edge $e_{0}$ is a 1-way edge oriented toward $v$. Then we define some edges $e_{1}, e_{2}, \ldots$ as follows: for all $i \geq 0$, if the origin of the 1-way edge $e_{i}$ is distinct from $u_{0}$ we define $e_{i+1}=\pi\left(e_{i}\right)$. We will now prove that there exists $i$ such that the origin of $e_{i}$ is $u_{0}$ (so that $e_{i}, e_{i-1}, \ldots, e_{0}$ is a directed path from $u_{0}$ to $v$ ). Suppose the contrary. In this case, there must exist integers $i<j$ such that the origin of $e_{j}$ is the end of $e_{i}$, and we consider the least such $j$. The edges $e_{i}, e_{i}+1, \ldots, e_{j}$ form a simple directed cycle $C$ of $H$, which is not the outer face of $H$. And since $\Omega$ has no counterclockwise directed cycle, except for the outer faces of $H$, the cycle $C$ is directed clockwise. The situation is represented in Figure 35(b). Let $u$ be the end of $e_{i}$ (also the origin of $e_{j}$ ). By definition of $e_{i}$, the edge of $G_{H}$ preceding $e_{i}$ around $u$ is part of a $\varphi$-positive directed path $P$ of $G_{H}$ from $u_{0}$ to $u$. Hence, the path $P$ must intersect the cycle $C$. We denote by $w$ the first vertex of $C$ on the path $P$ from $u_{0}$ to $u$, and by $e_{k}$ the edge of $C$ with origin $w$ (with $i \leq k<j$ ). Note that the directed path $P$ arrives at $w$ between $e_{k+1}=\pi\left(e_{k}\right)$ and $e_{k}$ in clockwise direction around $w$. This is impossible by definition of $\pi$. This completes the proof that there is a directed path from $u_{0}$ to $v$ in $\Omega$. Hence the hyperorientation $\Omega$ is accessible from every outer vertex of $H$.

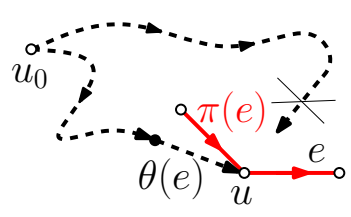

(a)

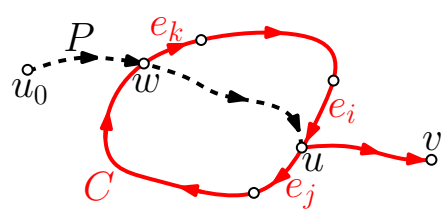

(b)

Figure 35. (a) Definition of the edge $\pi(e)$, for a 1-way edge $e$ of $H$ with origin $u \neq u_{0}$. The $\varphi$-positive paths of $G_{H}$ are represented in dashed lines. (b) The cycle $C=\left\{e_{i}, e_{i+1}, \ldots, e_{j}\right\}$ of $H$, and the $\varphi$-positive path $P$ of $G_{H}$ (represented in dashed line).

Uniqueness. Suppose that $\widetilde{\Omega}$ is a $\sigma$-weighted hyperorientation of $H$ which is minimal and accessible from every outer vertex of $H$. We want to prove that $\widetilde{\Omega}=\Omega$. It suffices to prove that the hyperflow $\widetilde{\varphi}:=\Gamma^{-1}(\widetilde{\Omega})$ of $G_{H}$ is equal to $\varphi$. By Lemma 63 , we know that $\widetilde{\varphi}$ is an $\alpha$-hyperflow of $G_{H}$. Hence by Lemma 59, it suffices to prove that $\widetilde{\varphi}$ is minimal. Suppose, by contradiction that $\widetilde{\varphi}$ is not minimal, and consider a simple $\widetilde{\varphi}$-positive counterclockwise cycle $D$ of $G_{H}^{\prime}$. We will now exhibit a counterclockwise directed cycle $C$ of $\widetilde{\Omega}$. For a vertex $x$ of $H$ on the cycle $D$ we consider the edge $a_{x}$ of $D$ oriented toward $x$, and the edge $e_{x}$ of $H$ following $a_{x}$ clockwise around $x$; see Figure 36(a). Since $\varphi\left(a_{x}\right)>0$, the edge $e_{x}$ is 1-way oriented toward $x$ in $\widetilde{\Omega}=\Gamma(\widetilde{\varphi})$. The origin $x^{\prime}$ of $e_{x}$ is either on the cycle $D$ or strictly inside $D$. If $x^{\prime}$ is strictly inside $D$, we consider a directed path of $\widetilde{\Omega}$ going from an outer vertex of $H$ to $x^{\prime}$ (we know that such a path exists since $\widetilde{\Omega} \in \mathcal{H}_{+}$). We extract from this path a directed path of $\widetilde{\Omega}$ starting at a vertex on the cycle $D$, staying strictly inside $D$ and ending at $x^{\prime}$. We denote by $Q_{x}$ the directed path of $\Omega$ made of $P_{x}$ followed by the edge $e_{x}$, with the convention that $P_{x}$ is empty if the origin $x^{\prime}$ of $e_{x}$ is on $D$. With this convention, for all $x$ of $H$ on the cycle $D$, the directed path $Q_{x}$ of $\widetilde{\Omega}$ starts at a vertex of $D$, stays strictly inside $D$ and ends at $x$. We now consider a vertex $x_{0}$ of $H$ on $D$, and for all $i \geq 0$ we denote by $x_{i+1}$ the origin of $Q_{x_{i}}$. The infinite path $\cup_{i=0}^{\infty} Q_{x_{i}}$ stays inside $D$ and must intersect itself. Let $n$ be the largest integer such that the path $Q=\cup_{i=0}^{n-1} Q_{x_{i}}$ from $x_{n}$ to $x_{0}$ is simple. Since $Q$ is simple, it cuts the interior of the cycle $D$ into two regions. Moreover,

\footnotetext{
${ }^{3}$ Our construction corresponds to the so-called leftmost path which has proved useful for other bijective results on maps.
} 
the edge $e_{x_{n}}$ is easily seen to be in the region on the left of $Q$. Therefore the path $\cup_{i=0}^{n} Q_{x_{i}}$ contains a counterclockwise cycle; see Figure 36 (b). This implies that $\widetilde{\Omega}$ is not minimal, a contradiction.

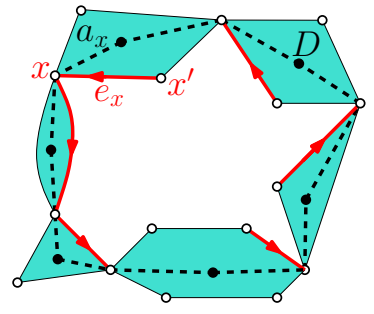

(a)

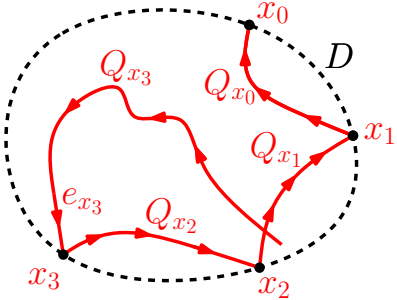

(b)

Figure 36. (a) The counterclockwise cycle $D$ of $G_{H}$ (dashed lines), a vertex $x$ of $H$ on $D$ and the 1-way edge $e_{x}$ of $H$. (b) The directed paths $Q_{x_{0}}, Q_{x_{1}}, \ldots$ of $H$ inside the cycle $D$ of $G_{H}$ and a counterclockwise cycle $C$ of $H$ contained in $\cup_{i=0}^{3} Q_{x_{i}}$.

Proof of Proposition 62. We now complete the proof of Proposition 62, By Lemma 65 there is a unique $\sigma$-weighted hyperorientation $\Omega$ which is both minimal and accessible from the outer vertices. In order to complete the proof of Proposition 62 we need to prove that $\Omega$ is in $\mathcal{H}_{+}$, that is, we need to prove that the outer face of $H$ is a clockwise directed cycle. Hence it suffices to prove that every outer edge of $H$ has positive weight (hence is 1-way).

Let $e_{0}$ be an outer edge of $H$. We denote by $w(a)$ the weight of a vertex, edge or face $a$ in $\Omega$. We want to prove $w\left(e_{0}\right)>0$. Let us first treat the case where $e_{0}$ is a loop. Let $f_{0}$ be the light outer face and let $f$ be the dark inner face incident to $e_{0}$. The light region $R=\left\{f_{0}, f\right\}$ satisfies $|\partial R|=\operatorname{deg}\left(f_{0}\right)-1$ and $\sigma(R)=\sigma\left(f_{0}\right)+\sigma(f)=\operatorname{deg}\left(f_{0}\right)+\sigma(f)$. Thus the $\sigma$-girth condition gives $-1>\sigma(f)$. Hence $w\left(e_{0}\right)=w(f)=-1-\sigma(f)>0$ as wanted.

We now suppose that $e_{0}$ is not a loop and want to prove $w\left(e_{0}\right)>0$. We consider the hypermap $H^{\prime}$ obtained from $H$ by adding two edges $e^{\prime}, e^{\prime \prime}$ with the same endpoints as $e_{0}$ in the outer light face of $H$ as indicated in Figure 37(a). In $H^{\prime}$, the edges $e_{0}$ and $e^{\prime}$ enclose an inner light face $f^{\prime}$ of degree 2 , while the edges $e^{\prime}$ and $e^{\prime \prime}$ enclose an inner dark face $f^{\prime \prime}$ of degree 2. Let

$$
\epsilon=\frac{1}{2} \min _{R}(|\partial R|-\sigma(R))
$$

where $R$ ranges over all light regions containing strictly at least one of the outer edges. Since $H$ satisfies the $\sigma$-girth condition, we have $\epsilon>0$. Let $f$ be the dark face incident to $e_{0}$ and let $\sigma^{\prime}$ be the charge function of $H^{\prime}$ defined by $\sigma^{\prime}(f)=\sigma(f)+\epsilon, \sigma^{\prime}\left(f^{\prime}\right)=2$, $\sigma^{\prime}\left(f^{\prime \prime}\right)=-2-\epsilon$, and $\sigma^{\prime}(a)=\sigma(a)$ for any vertex or face $a \notin\left\{f, f^{\prime}, f^{\prime \prime}\right\}$ of $H^{\prime}$.

Claim 66. The charge function $\sigma^{\prime}$ fits $H^{\prime}$.

Proof. It is easy to see that the charge $\sigma^{\prime}(v)=\sigma(v)$ of every vertex is positive, the charge $\sigma^{\prime}\left(f_{0}\right)=\sigma\left(f_{0}\right)$ of the light outer face $f_{0}$ is $\operatorname{deg}\left(f_{0}\right)$, and $\sigma_{\text {total }}^{\prime}=\sigma_{\text {total }}=0$. It remains to prove that $H^{\prime}$ satisfies the $\sigma^{\prime}$-girth condition. Let $R^{\prime}$ be a light region of $H^{\prime}$. We need to prove $\left|\partial R^{\prime}\right| \geq \sigma^{\prime}\left(R^{\prime}\right)$, with strict inequality if $R^{\prime}$ strictly contains an outer edge.

First suppose that $f^{\prime \prime} \in R^{\prime}$. In this case $f_{0}, f^{\prime} \in R^{\prime}$ (because $R^{\prime}$ is a light region). Let $R$ be the light region of $H$ obtained from $R^{\prime}$ by removing $f^{\prime}, f^{\prime \prime}$. If $f \in R$, then $R$ strictly contains the outer edge $e_{0}$ so that

$$
\left|\partial R^{\prime}\right|=|\partial R| \geq \sigma(R)+2 \epsilon=\sigma^{\prime}\left(R^{\prime}\right)+2 \epsilon,
$$

while if $f \notin R$, then

$$
\left|\partial R^{\prime}\right|=|\partial R| \geq \sigma(R)=\sigma^{\prime}\left(R^{\prime}\right)+\epsilon .
$$




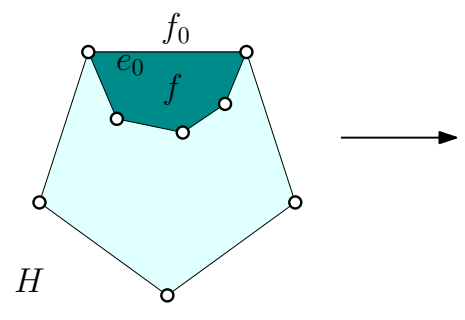

(a)

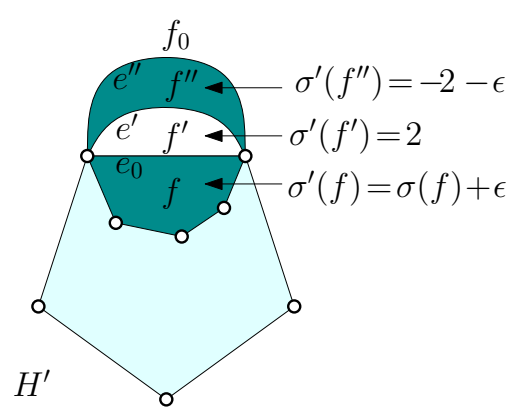

$H^{\prime}$

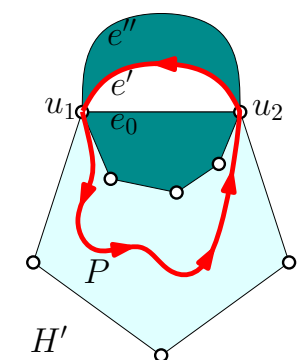

(b)

Figure 37. (a) (a) The hypermap $H^{\prime}$ obtained from $H$ by adding two edges $e^{\prime}, e^{\prime \prime}$ with the same endpoints as $e_{0}$ (and conveniently assigning charges for the new faces and the dark face incident to $e_{0}$ ). (b) If $e^{\prime}$ was 1-way, by the accessibility properties of $\mathcal{H}_{+}$, it would yield a $P$ forming with $e^{\prime}$ a counterclockwise cycle (shown in red), a contradiction. .

Next suppose that $f^{\prime \prime} \notin R^{\prime}$ and $f \notin R^{\prime}$. If $f^{\prime} \notin R^{\prime}$ then $R^{\prime}$ is a light region of $H$ and we get

$$
\left|\partial R^{\prime}\right| \geq \sigma\left(R^{\prime}\right)=\sigma^{\prime}\left(R^{\prime}\right),
$$

with strict inequality if $R^{\prime}$ strictly contains an outer edge. If $f^{\prime} \in R^{\prime}$ then we consider the light region $R$ of $H$ obtained from $R^{\prime}$ by removing $f^{\prime}$. We get

$$
\left|\partial R^{\prime}\right|=|\partial R|+2 \geq \sigma\left(R^{\prime}\right)+2=\sigma^{\prime}(R),
$$

with strict inequality if $R^{\prime}$ (hence $R$ ) strictly contains an outer edge.

Lastly suppose that $f^{\prime \prime} \notin R^{\prime}$ and $f \in R^{\prime}$. In this case $f^{\prime} \in R^{\prime}$. If $f_{0} \in R$, we consider the light region $R$ of $H$ obtained from $R^{\prime}$ by removing $f^{\prime}$. Since $R$ strictly contains the outer edge $e_{0}$ we get

$$
\left|\partial R^{\prime}\right|=|\partial R|+2 \geq \sigma(R)+2 \epsilon+2=\sigma^{\prime}\left(R^{\prime}\right)+\epsilon
$$

If $f_{0} \notin R$, then we consider the light region $R$ of $H$ obtained from $R^{\prime}$ by removing $f^{\prime}$ and adding $f_{0}$. Since $R$ strictly contains the outer edge $e_{0}$ we get

$$
\left|\partial R^{\prime}\right|=|\partial R|+2-\operatorname{deg}\left(f_{0}\right) \geq \sigma(R)+2 \epsilon+2-\operatorname{deg}\left(f_{0}\right)=\sigma^{\prime}\left(R^{\prime}\right)+\epsilon .
$$

Since $H^{\prime}$ satisfies the $\sigma^{\prime}$-girth condition, Lemma 65 implies that $H^{\prime}$ admits a $\sigma^{\prime}$-weighted hyperorientation $\Omega^{\prime}$ which is minimal and accessible from every outer vertex. Let $e_{1}$ be the edge preceding $e_{0}$ in clockwise order around $f$. We define a weighted hyperorientation $\widetilde{\Omega}$ of $H$ by setting $\widetilde{w}\left(e_{0}\right)=w^{\prime}\left(e_{0}\right)+w^{\prime}\left(e^{\prime \prime}\right), \widetilde{w}\left(e_{1}\right)=w^{\prime}\left(e_{1}\right)+w^{\prime}\left(e^{\prime}\right)$, and $\widetilde{w}(e)=w^{\prime}(e)$ for all edge $e \neq e_{0}, e_{1}$ of $H$ (as usual the edge in $\widetilde{\Omega}$ are 1-way if they have positive weight and 0 -way otherwise). We denote by $w^{\prime}(a)$ and $\widetilde{w}(a)$ respectively the weight of a vertex, edge or face $a$ in $\Omega^{\prime}$ and $\widetilde{\Omega}$. Recall that all the weights $w^{\prime}(a)$ are non-negative, hence the weights $\widetilde{\Omega}(a)$ are non-negative.

Claim 67. The hyperorientation $\widetilde{\Omega}$ is $\sigma$-weighted. Moreover $\widetilde{w}\left(e_{0}\right)>0$.

Proof. It is easily seen that the weight of every vertex is the same in $\Omega^{\prime}$ and $\widetilde{\Omega}$ (also for the endpoints of $e_{0}$ ). Moreover the weight of every face of $H$ is the same in $\Omega^{\prime}$ and $\widetilde{\Omega}$ except for the dark face $f$. For the dark face $f$ we have

$\widetilde{w}(f)=w^{\prime}(f)+w^{\prime}\left(e^{\prime}\right)+w^{\prime}\left(e^{\prime \prime}\right)=w^{\prime}(f)+w^{\prime}\left(f^{\prime \prime}\right)=-\sigma^{\prime}(f)-\operatorname{deg}(f)-\sigma^{\prime}\left(f^{\prime \prime}\right)-2=-\sigma(f)-\operatorname{deg}(f)$, as wanted. Since $\Omega^{\prime}$ is $\sigma^{\prime}$-weighted, this shows that $\widetilde{\Omega}$ is $\sigma$-weighted. 
It remains to show that $\widetilde{w}\left(e_{0}\right)>0$. It suffices to show $w^{\prime}\left(e^{\prime \prime}\right)>0$. Suppose by contradiction that $w^{\prime}\left(e^{\prime \prime}\right)=0$. In this case $w^{\prime}\left(e^{\prime}\right)=w^{\prime}\left(f^{\prime \prime}\right)=\epsilon>0$, so that $e^{\prime}$ is 1-way and $e^{\prime \prime}$ is 0 -way in the hyperorientation $\Omega^{\prime}$. Let $u_{2}$ and $u_{1}$ be the origin and end of $e^{\prime}$ as indicated in Figure $37(\mathrm{~b})$. Since $\Omega^{\prime}$ is accessible from the outer vertex $u_{1}$, there is a directed path $P$ from $u_{1}$ to $u_{2}$. This path does not use the outer edge $e^{\prime \prime}$ which is 0 -way, hence the path $P$ together with $e^{\prime}$ form a counterclockwise directed cycle; see Figure $37(\mathrm{~b})$. This is a contradiction since $\Omega^{\prime}$ is minimal.

We know $w\left(\widetilde{e_{0}}\right)>0$ and want to prove $w\left(e_{0}\right)>0$. For this we use Lemma 61. Since the hyperorientation $\widetilde{\Omega}$ of $H$ is $\sigma$-weighted, we know by Lemma 63 that the hyperflow $\widetilde{\varphi}=$ $\Gamma^{-1}(\widetilde{\Omega})$ is an $\alpha$-hyperflow of $G_{H}$. Let $a_{0}$ be the edge of $G_{H}$ preceding $e_{0}$ clockwise around the outer vertex $u_{2}$ of $H$. By definition of $\Gamma, \widetilde{\varphi}\left(a_{0}\right)=\widetilde{w}\left(e_{0}\right)>0$. Hence by Lemma 61, the minimal $\alpha$-hyperflow of $G_{H}$ satisfies $\varphi\left(a_{0}\right)>0$. Moreover the hyperorientation $\Omega$ is equal to $\Gamma(\varphi)$ (see the proof of Lemma 65). Thus $w\left(e_{0}\right)=\varphi\left(a_{0}\right)>0$. This completes the proof of Proposition 62 .

10.4. Proof of Theorem 26. In this section we complete the proof of Theorem 26. We consider a light-rooted hypermap $H$, and a charge function $\sigma$ fitting $H$. We want to prove that $H$ admits a unique $\sigma$-weighted hyperorientation in $\mathcal{H}_{+}$. Our strategy is as follows. First, we will construct a related hypermap $H^{(k)}$ and a fitting charge function $\sigma^{(k)}$ satisfying the condition of Proposition 62 . This grants the existence of a unique $\sigma^{(k)}$-weighted hyperorientation $\Omega^{(k)}$ in $\mathcal{H}_{+}$for $H^{(k)}$. We will then construct from $\Omega^{(k)}$ a hyperorientation $\Omega$ of $H$, and prove that it is the unique $\sigma$-weighted hyperorientation of $H$ in $\mathcal{H}_{+}$.

Let $k$ be an integer greater than

$$
1+\left|E_{0}\right|+\sum_{a \in A}|\sigma(a)|
$$

where $E_{0}$ is the set of edges of $H$, and $A$ is the set of all vertices and faces of $H$. Let $H_{k}$ be the hypermap obtained from $H$ by subdividing every edge into a path of length $k$. Hence, every face of degree $\delta$ of $H$ corresponds to a face of degree $k \delta$ of $H_{k}$. We now consider a hypermap $H^{(k)}$ obtained by adding a dark face of degree $k(k-1) \delta$, called a sea-star, inside each inner light face of degree $k \delta$ of $H_{k}$; see Figure 38 . More precisely, $H^{(k)}$ is obtained by adding the sea-stars inside the inner light faces of $H_{k}$ in such a way that every inner light faces of $H^{(k)}$ has degree $k$ and is incident to one edge of $H_{k}$ and $k-1$ edges of a sea-star. We call sea-edges the edges of $H^{(k)}$ incident to sea-stars. For an edge $e=(u, v)$ of $H_{k}$, we call sea-arc associated with $e$ the path of $H^{(k)}$ made of the $k-1$ sea-edges around the face of $H^{(k)}$ incident to $e$. For an edge $e=(u, v)$ of $H$, we call sea-path associated with $e$ the path of $H^{(k)}$ from $u$ to $v$ (of length $k(k-1)$ ) made of the $k$ sea-arcs associated with the edges of $H_{k}$ subdividing $e$.

We define a charge function $\sigma^{(k)}$ of $H^{(k)}$ as follows:

- $\sigma^{(k)}(v)=k \sigma\left(v^{\prime}\right)$ if $v$ is a vertex of $H^{(k)}$ corresponding to a vertex $v^{\prime}$ of $H$ and $\sigma^{(k)}(v)=k$ otherwise,

- $\sigma^{(k)}(f)=\operatorname{deg}(f)$ if $f$ is a light face (hence $\sigma^{(k)}(f)=k$ for every inner light face $f$ ),

- $\sigma^{(k)}(f)=k \sigma\left(f^{\prime}\right)-k^{2} \delta+k \delta$ if $f$ is a dark face of $H^{(k)}$ of degree $k \delta$ corresponding to a dark face $f^{\prime}$ of $H$ (of degree $\delta$ ),

- $\sigma^{(k)}(f)=k \sigma\left(f^{\prime}\right)-k^{2}(k-1) \delta$ if $f$ is a sea-star of degree $k(k-1) \delta$ corresponding to a light face $f^{\prime}$ of $H$ (of degree $\delta$ ).

Claim 68. The charge function $\sigma^{(k)}$ fits $H^{(k)}$.

Proof. First observe that the charge $\sigma^{(k)}(v)=k \sigma(v)$ is positive for every vertex $v$, and the charge of the outer face is equal to its degree. We now show that $\sigma_{\text {total }}^{(k)}=0$. Let $V_{0}, E_{0}, F_{0}$, $S_{0}$, and $K_{0}$ be respectively the set of vertices, edges, light faces, sea-stars, and non-sea-star 

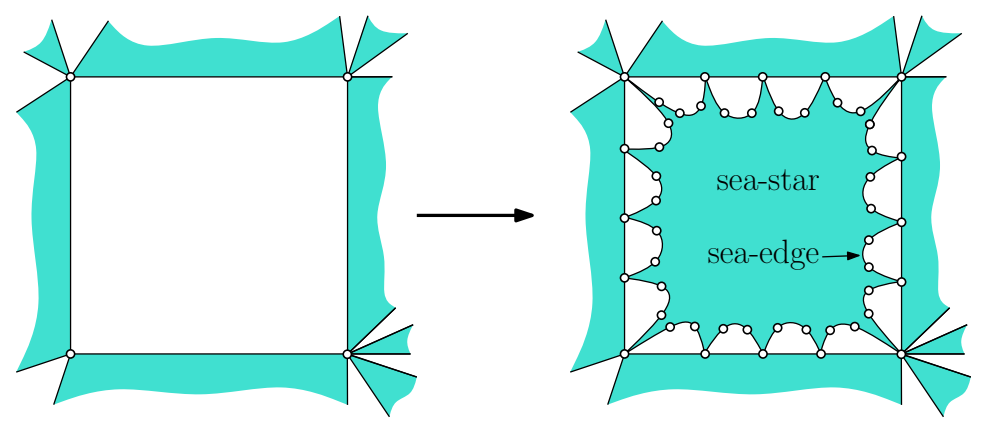

Figure 38. Left: A face $f$ of $H$. Right: the face $f$ after subdividing each edge into a path of length $k=4$, and adding the sea-star of $H^{(k)}$ inside $f$.

dark faces of $H^{(k)}$. Let $E_{1}$ and $E_{2}$ be respectively the set of edges of $H^{(k)}$ incident to sea-stars, and to non-sea-star dark faces of $H^{(k)}$. We have

$$
\begin{aligned}
\sigma_{\text {total }}^{(k)}= & \sum_{v \in V_{0}} \sigma^{(k)}(v)+\sum_{f \in F_{0}} \sigma^{(k)}(f)+\sum_{f \in S_{0}} \sigma^{(k)}(f)+\sum_{f \in K_{0}} \sigma^{(k)}(f) \\
= & k\left(\left|V_{0}\right|-\left|V_{0}^{\prime}\right|+\sum_{v^{\prime} \in V_{0}^{\prime}} \sigma\left(v^{\prime}\right)\right)+k\left(\left|F_{0}\right|+\operatorname{deg}\left(f_{0}^{\prime}\right)-1\right) \\
& +k\left(-\left|E_{1}\right|+\sum_{f^{\prime} \in S_{0}^{\prime}} \sigma\left(f^{\prime}\right)\right)+k\left(\left|E_{0}^{\prime}\right|-\left|E_{2}\right|+\sum_{f^{\prime} \in K_{0}^{\prime}} \sigma\left(f^{\prime}\right)\right) \\
= & k\left(\left|V_{0}\right|+\left|F_{0}\right|-\left|E_{0}\right|-\left|V_{0}^{\prime}\right|+\left|E_{0}^{\prime}\right|-1+\sigma_{\text {total }}\right)
\end{aligned}
$$

where $f_{0}^{\prime}$ is the outer face of $H$, and $V_{0}^{\prime}, E_{0}^{\prime}, S_{0}^{\prime}, K_{0}^{\prime}$ are respectively the set of vertices, edges, inner light faces, and dark faces of $H$ (the last identity uses $\left|E_{0}\right|=\left|E_{1}\right|+\left|E_{2}\right|$ and $\left.\sigma\left(f_{0}^{\prime}\right)=\operatorname{deg}\left(f_{0}^{\prime}\right)\right)$. The Euler relation gives

$$
\left|V_{0}\right|+\left|F_{0}\right|-\left|E_{0}\right|=-\left|S_{0}\right|-\left|K_{0}\right|+2=-\left|S_{0}^{\prime}\right|-\left|K_{0}^{\prime}\right|+2=\left|V_{0}^{\prime}\right|-\left|E_{0}^{\prime}\right|+1,
$$

because $\left|S_{0}\right|=\left|S_{0}^{\prime}\right|$ and $\left|K_{0}\right|=\left|K_{0}^{\prime}\right|$. Moreover $\sigma_{\text {total }}=0$, hence $\sigma_{\text {total }}^{(k)}=0$.

It remains to prove that $H^{(k)}$ satisfies the $\sigma^{(k)}$-girth condition. Let $R$ be a light region of $H^{(k)}$. We want to prove $|\partial R| \geq \sigma^{(k)}(R)$ with strict inequality if one of the outer edges is strictly contained in $R$. By Lemma 32 we can assume that the light region $R$ is simply connected. Let $V, E, F, S$, and $K$ be respectively the set of vertices strictly inside $R$, edges strictly inside $R$, light faces inside $R$, sea-stars inside $R$, and non-sea-star dark faces inside $R$. Similarly as in the above computation of $\sigma_{\text {total }}^{(k)}$, we have

$$
\begin{aligned}
\sigma^{(k)}(R) & =\sum_{v \in V} \sigma^{(k)}(v)+\sum_{f \in F} \sigma^{(k)}(f)+\sum_{f \in K} \sigma^{(k)}(f)+\sum_{f \in S} \sigma^{(k)}(f) \\
& =k\left(|V|+|F|-|E|-\left|V^{\prime}\right|+\left|E^{\prime}\right|+\mathbf{1}_{f_{0} \in R} \cdot\left(\operatorname{deg}\left(f_{0}^{\prime}\right)-1\right)+\sum_{a \in V^{\prime} \cup S^{\prime} \cup K^{\prime}} \sigma(a)\right),
\end{aligned}
$$

where $f_{0}$ is the outer face of $H^{(k)}, V^{\prime}$ is the set of vertices of $H$ corresponding to vertices of $H^{(k)}$ in $V, S^{\prime}$ is the set of inner light faces of $H$ corresponding to sea-stars in $S, K^{\prime}$ is the set of dark faces of $H$ corresponding to dark faces in $K$, and $E^{\prime}$ is the set of edges of $H$ incident to faces in $K^{\prime}$. Since $R$ is simply connected, the Euler relation gives $|V|+|F|-|E|=$ $-\left|S^{\prime}\right|-\left|K^{\prime}\right|+1$, hence

$$
\sigma^{(k)}(R)=k\left(\left|E^{\prime}\right|-\left|V^{\prime}\right|-\left|S^{\prime}\right|-\left|K^{\prime}\right|+1+\mathbf{1}_{f_{0} \in R}\left(\operatorname{deg}\left(f_{0}^{\prime}\right)-1\right)+\sum_{a \in V^{\prime} \cup S^{\prime} \cup K^{\prime}} \sigma(a)\right) .
$$


(In particular, by the choice of $k, \sigma^{(k)}(R)<k(k-1)$.)

We now prove $|\partial R| \geq \sigma^{(k)}(R)$ with strict inequality if one of the outer edges is strictly contained in $R$. Suppose first that $R$ contains an inner light face $f$ but contains none of the two dark faces incident to $f$. Since $R$ is connected, we have $R=\{f\}$ and $|\partial R|=k=\sigma(R)$. Next, suppose that $R$ contains an inner light face and the incident non-sea-star dark face $f$, but not the incident sea-star $s$. Since $f \in R$ all the incident light faces are in $R$ (because $R$ is a light region) hence $C$ contains an entire sea path. Thus $|\partial R| \geq k(k-1)>\sigma^{(k)}(R)$ by the choice of $k$. Lastly suppose that for every light face $f$ in $R$, the sea-star incident to $f$ is also in $R$. In this case, we consider the light region $R^{\prime}$ of $H$ defined by $R^{\prime}=K^{\prime} \cup S^{\prime}$ if $f_{0} \notin R$ and $R^{\prime}=K^{\prime} \cup S^{\prime} \cup\left\{f_{0}^{\prime}\right\}$ if $f_{0} \in R$. We have $|\partial R|=k\left|\partial R^{\prime}\right|$. Moreover $V^{\prime}, E^{\prime}, S^{\prime}$, and $K^{\prime}$ are respectively the sets of vertices strictly inside $R^{\prime}$, edges strictly inside $R^{\prime}$, inner light faces inside $R^{\prime}$, and dark faces inside $R^{\prime}$, so that the Euler relation gives

$$
\left|E^{\prime}\right|-\left|V^{\prime}\right|-\left|S^{\prime}\right|-\left|K^{\prime}\right|+1-\mathbf{1}_{f_{0} \in R^{\prime}}=0 .
$$

Hence, using $\operatorname{deg}\left(f_{0}^{\prime}\right)=\sigma\left(f_{0}^{\prime}\right)$ we get

$$
\sigma^{(k)}(R)=k\left(\mathbf{1}_{f_{0} \in R^{\prime}} \sigma\left(f_{0}^{\prime}\right)+\sum_{a \in V^{\prime} \cup S^{\prime} \cup K^{\prime}} \sigma(a)\right)=k \sigma\left(R^{\prime}\right) .
$$

Thus $|\partial R|=k\left|\partial R^{\prime}\right| \geq k \sigma\left(R^{\prime}\right)=\sigma^{(k)}(R)$ with strict inequality if one of the outer edges is strictly contained in $R$.

By Claim 68 and Proposition 62 the hypermap $H^{(k)}$ admits a unique $\sigma^{(k)}$-weighted hyperorientation $\Omega^{(k)}$ in $\mathcal{H}_{+}$. We now establish a few properties of $\Omega^{(k)}$. We denote by $w(a)$ the weight of an edge or face $a$ of $H^{(k)}$ in the hyperorientation $\Omega^{(k)}$. Note that all the weights are non-negative because every light face of $H^{(k)}$ has degree equal to its charge. Let $a$ be an inner edge of $H_{k}$ and let $P$ be the associated sea-arc. The $k-2$ first edges of $P$ are forced to have weight $k$, and we denote by $w^{\prime}(a)$ the weight of the last edge of $P$; see Figure 39. Let $f$ be an inner light face of $H$ of degree $\delta$, let $f_{k}$ be the corresponding light face of $H_{k}$ and let $s$ be the corresponding sea-star of $H^{(k)}$. For the edges $e_{1}, \ldots, e_{k \delta}$ incident to $f_{k}$ we get

$$
\begin{aligned}
w^{\prime}\left(e_{1}\right)+\ldots+w^{\prime}\left(e_{k \delta}\right) & =w(s)-k^{2}(k-2) \delta=-\sigma^{(k)}(s)-\operatorname{deg}(s)-k^{2}(k-2) \delta \\
& =-k \sigma(f)+k^{2}(k-1) \delta-k(k-1) \delta-k^{2}(k-2) \delta \\
& =-k \sigma(f)+k \delta .
\end{aligned}
$$

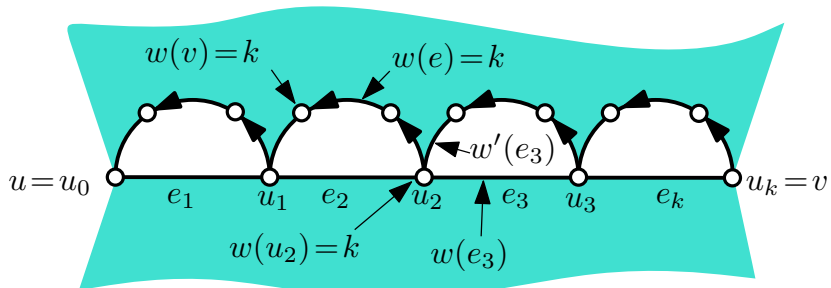

FIGURE 39. The sea-path associated with an inner edge $e=(u, v)$ of $H$ for $k=4$. The edges with weights $w\left(e_{i}\right)$ and $w^{\prime}\left(e_{i}\right)$ are indicated for $i=3$.

Claim 69. Let $e$ be an inner edge of $H$. Let $e_{1}, \ldots, e_{k}$ be the edges of $H_{k}$ subdividing $e$ in clockwise order around the incident dark face. There exists $j \in\{1, \ldots, k\}$ such that $w\left(e_{i}\right)=$ $k$ for all $i<j, w\left(e_{i}\right)=0$ for all $i>j$. Moreover $w^{\prime}\left(e_{1}\right)=0$, and $w^{\prime}\left(e_{i+1}\right)=k-w\left(e_{i}\right)$ for all $i \in\{1, \ldots, k-1\}$.

The situation described by Claim 69 is represented in Figure 40 (first line). 
Proof. We let $e_{i}=\left(u_{i-1}, u_{i}\right)$, with $u_{0}=u$, and $u_{k}=v$. Since for all $i \in\{1, \ldots, k-1\}$ the weight of the vertex $u_{i}$ is $k$, we get $w^{\prime}\left(e_{i+1}\right)=k-w\left(e_{i}\right)$. Since $\Omega$ is minimal, the weights $w\left(e_{i}\right)$ and $w^{\prime}\left(e_{i}\right)$ cannot both be positive (otherwise the incident light face would be oriented counterclockwise). Thus if $w\left(e_{i}\right) \neq k$ for $i<k$, then $w^{\prime}\left(e_{i+1}\right) \neq 0$, hence $w\left(e_{i+1}\right)=0$. This proves the existence of $j \in\{1, \ldots, k\}$ such that $w\left(e_{i}\right)=k$ for all $i<j$, and $w\left(e_{i}\right)=0$ for all $i>j$. Lastly, suppose by contradiction that $w^{\prime}\left(e_{1}\right)>0$. In this case $w\left(e_{1}\right)=0$, and $w^{\prime}\left(e_{i}\right)=k$ for all $i \in\{2, \ldots, k\}$. Thus

$$
w^{\prime}\left(e_{1}\right)+\ldots+w^{\prime}\left(e_{k}\right)=w^{\prime}\left(e_{1}\right)+k(k-1)>k(k-1) .
$$

By our choice of $k$ this contradicts (7).

We now associate with the weighted hyperorientation $\Omega^{(k)}$ of $H^{(k)}$ a weighted hyperorientation $\bar{\Omega}$ of $H$; see Figure 40 (note that, by these rules, an edge $e \in \bar{\Omega}$ is 1-way iff the associated $j$ defined in Claim 69 is equal to $k$ ). Let $e$ be an edge of $H$ and let $e_{1}, \ldots, e_{k}$ be the edges of $H_{k}$ subdividing $e$ in clockwise order around the incident dark face. We define the weight $\bar{w}(e)$ of $e$ in $\bar{\Omega}$ to be

$$
\bar{w}(e)=\frac{\sum_{i=1}^{k} w\left(e_{i}\right)}{k}-(k-1)
$$

and we orient $e$-way if the weight is positive and 0 -way otherwise. Note that for any outer edge $e$, the edges $e_{1}, \ldots, e_{k-1}$ are all 1-way of weight $k$ and $e_{k}$ is also 1-way (because $\left.\Omega^{(k)} \in \mathcal{H}_{+}\right)$, so that

$$
\bar{w}(e)=w\left(e_{k}\right) / k>0,
$$

hence $e$ is 1 -way.
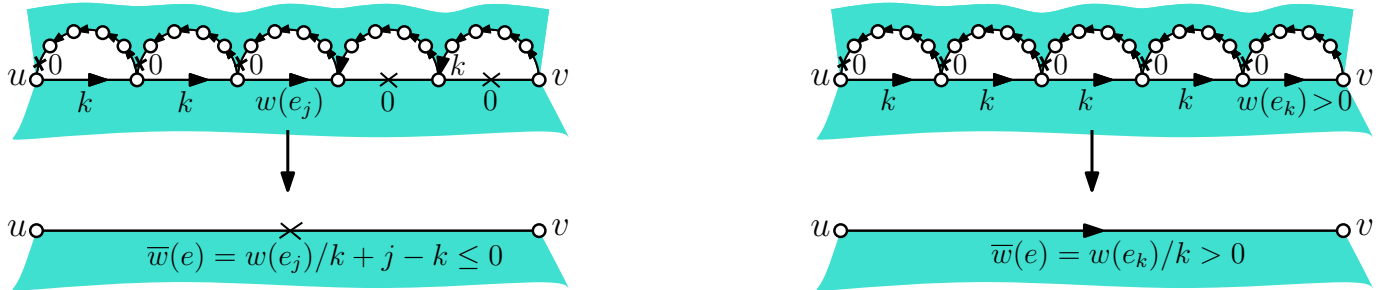

FigurE 40. Top part: the possible configurations of weights along a seapath in the hyperorientation $\Omega^{(k)}$ of $H^{(k)}$, as described by Claim 69 . Bottom part: the corresponding weight in the hyperorientation $\bar{\Omega}$ of $H$. In the case $j<k$ (left) one gets $\bar{w}(e)=\frac{\left(\sum_{i=1}^{k} w\left(e_{i}\right)\right)}{k}-(k-1)=w\left(e_{j}\right) / k-(j-1) \leq$ 0 , while in the case $j=k$ one gets $\bar{w}(e)=w\left(e_{k}\right) / k>0$.

We will now complete the proof of Theorem 26 by proving the following claim.

Claim 70. The hyperorientation $\bar{\Omega}$ is the unique $\sigma$-weighted hyperorientation of $H$ in $\mathcal{H}_{+}$.

Proof. We denote by $w(a)$ (resp. $\bar{w}(a)$ ) the weight of a vertex, edge or face $a$ in the hyperorientation $\Omega^{(k)}$ of $H^{(k)}$ (resp. $\bar{\Omega}$ of $H$ ). If $e$ is an inner edge of $H$, adopting the notation of Claim 69 gives

$$
\bar{w}(e)=\frac{1}{k}\left(w\left(e_{k}\right)-\left(\sum_{i=1}^{k} w^{\prime}\left(e_{i}\right)\right)\right) .
$$

Moreover, since $w\left(e_{k}\right)>0$ if and only if $\sum_{i=1}^{k} w^{\prime}\left(e_{i}\right)=0$, we get

$$
\max (\bar{w}(e), 0)=\frac{w\left(e_{k}\right)}{k},
$$


and

$$
\min (\bar{w}(e), 0)=-\frac{\sum_{i=1}^{k} w^{\prime}\left(e_{i}\right)}{k} .
$$

Now if $e$ is an outer edge $e$, then the edges $e_{1}, \ldots, e_{k-1}$ are all 1-way of weight $k$ and $e_{k}$ is also 1-way (because $\Omega^{(k)} \in \mathcal{H}_{+}$), so that

$$
\max (\bar{w}(e), 0)=\bar{w}(e)=w\left(e_{k}\right) / k,
$$

and $e$ is oriented 1 -way in $\Omega^{(k)}$.

We will now prove that $\bar{\Omega}$ is $\sigma$-weighted. Let $u$ be a vertex of $H$. We observe that Claim 69 (more precisely, the statement $w^{\prime}\left(e_{1}\right)=0$ in this claim) implies that no sea-edge is oriented 1-way toward $u$. Thus, the weight $w(u)$ is equal to the sum of the weights of the edges of $H_{k}$ oriented toward $u$. Hence (8) gives

$$
\bar{w}(u)=\sum_{e \text { oriented toward } u \text { in } H} \max (\bar{w}(e), 0)=\sum_{e^{\prime} \text { oriented toward } u \text { in } H_{k}} \frac{w\left(e^{\prime}\right)}{k}=\frac{w(u)}{k}=\frac{\sigma^{(k)}(u)}{k}=\sigma(u),
$$

as wanted. Now let $f$ be a light inner face of $H$ of degree $\delta$, and let $f^{\prime}$ be the corresponding face in $H_{k}$. By $(9)$, the weight of $f$ in $\bar{\Omega}$ is

$$
\bar{w}(f)=\sum_{e \text { incident to } f \text { in } H} \min (\bar{w}(e), 0)=-\sum_{e_{i} \text { incident to } f^{\prime} \text { in } H_{k}} \frac{w^{\prime}\left(e_{i}\right)}{k} .
$$

Hence, (7) gives $\bar{w}(f)=\sigma(f)-\delta$ as wanted. Now, let $f$ be a dark inner face of $H$ of degree $\delta$, and let $f^{\prime}$ be the corresponding face of $H^{(k)}$. The weight of $f$ in $\bar{\Omega}$ is

$$
\begin{aligned}
\bar{w}(f) & =\sum_{e \text { incident to } f} \bar{w}(e)=\left(\sum_{e^{\prime} \text { incident to } f^{\prime}} \frac{w\left(e^{\prime}\right)}{k}\right)-(k-1) \delta=\frac{w\left(f^{\prime}\right)}{k}-(k-1) \delta \\
& =\frac{-\sigma^{(k)}\left(f^{\prime}\right)-\operatorname{deg}\left(f^{\prime}\right)}{k}-(k-1) \delta=-\sigma(f)-\delta,
\end{aligned}
$$

as wanted. Thus $\bar{\Omega}$ is $\sigma$-weighted.

Next we prove that $\bar{\Omega}$ is in $\mathcal{H}_{+}$. As noted above, the outer edges of $H$ are 1-way in $\bar{\Omega}$ (hence they form a clockwise directed cycle), hence it remains to prove that $\bar{\Omega}$ is minimal and accessible from outer vertices. For an edge $e=(u, v)$ of $H$ we consider the subgraph $G_{e}$ of $H^{(k)}$ made of the path subdividing $e$ together with the sea-path associated with $e$. In the hyperorientation $\Omega^{(k)}$ of $G_{e}$ the sea-path cannot be used to go from $u$ to $v$ nor from $v$ to $u$ because of Claim 69 (more precisely, the statement $w^{\prime}\left(e_{1}\right)=0$ in this claim). Moreover, the path subdividing $e$ is oriented from $u$ to $v$ in $\Omega^{(k)}$ if and only if $e$ is oriented 1-way from $u$ to $v$ in the hyperorientation $\bar{\Omega}$; see Figure 40 . Thus for any vertices $v_{1}, v_{2}$ of $H$, there is a directed path from $v_{1}$ to $v_{2}$ in the hyperorientation $\Omega^{(k)}$ of $H^{(k)}$ if and only if there is a directed path from $v_{1}$ to $v_{2}$ in the hyperorientation $\bar{\Omega}$ of $H$. Since $\Omega^{(k)}$ is in $\mathcal{H}_{+}$, we conclude that in the hyperorientation $\bar{\Omega}$ of $H$ every vertex is accessible from every outer vertex. Moreover any simple directed cycle in the hyperorientation $\bar{\Omega}$ of $H$ corresponds to a directed simple cycle in the hyperorientation $\Omega^{(k)}$ of $H^{(k)}$. Hence the minimality of the hyperorientation $\Omega^{(k)}$ implies the minimality of $\bar{\Omega}$. Thus $\bar{\Omega}$ is in $\mathcal{H}_{+}$.

Lastly we prove that there does not exist a $\sigma$-weighted hyperorientation $\bar{\Omega}^{\prime} \in \mathcal{H}_{+}$of $H$ distinct from $\bar{\Omega}$. Suppose the contrary. By inverting the construction represented in Figure 40 (using the fact that $\bar{w}(e) \geq-k+1$ by our choice of $k$ ), one can associate with $\bar{\Omega}^{\prime}$ a hyperorientation $\Omega^{\prime} \neq \Omega^{(k)}$ of $H^{(k)}$ satisfying the properties described in Claim 69 . It is then easy to see using the same relations as above that $\Omega^{\prime}$ is $\sigma^{(k)}$-weighted. Moreover, by the properties highlighted in the previous paragraph, it is easily seen that $\Omega^{\prime}$ is minimal and accessible from every outer vertex. Thus we obtain a $\sigma^{(k)}$-weighted hyperorientation $\Omega^{\prime} \neq \Omega^{(k)}$ in $\mathcal{H}_{+}$. This is impossible because this contradicts the uniqueness property of Proposition 62 
Claim 70 proves that if a charge function $\sigma$ fits a light-rooted hypermap $H$, then $H$ admits a unique $\sigma$-weighted hyperorientation in $\mathcal{H}_{+}$. This, together with Lemma 56 , completes the proof of Theorem 26

10.5. Proof of Theorems 25 and 27. In this section we prove Theorems 25 and 27 by a reduction to Theorem 26 .

We start with Theorem 25. Let $H$ be a dark-rooted hypermap with a simple outer face, and let $\sigma$ be a charge function fitting $H$. We want to prove that $H$ admits a unique $\sigma$ weighted hyperorientation in $\mathcal{H}_{-}$. Let $H^{\prime}$ be the light-rooted hypermap obtained from $H$ by adding a dark face of degree 2 along each of the outer edges of $H$, and changing the outer face color into light, as indicated in Figure 41. Observe that the outer faces $f_{0}$ of $H$ and $f_{0}^{\prime}$ of $H^{\prime}$ have the same degree. We call outer digons of $H^{\prime}$ the added dark faces. We define a charge function $\sigma^{\prime}$ of $H^{\prime}$ by setting $\sigma^{\prime}\left(f_{0}^{\prime}\right)=\operatorname{deg}\left(f_{0}^{\prime}\right), \sigma^{\prime}(f)=-3$ if $f$ is an outer digon, $\sigma^{\prime}(v)=1$ if $v$ is an outer vertex, and $\sigma^{\prime}(a)=\sigma(a)$ if $a$ is any inner vertex or inner face of $H$.

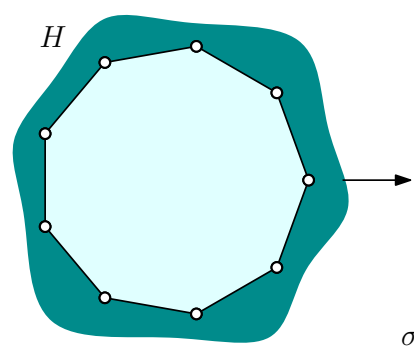

(a)

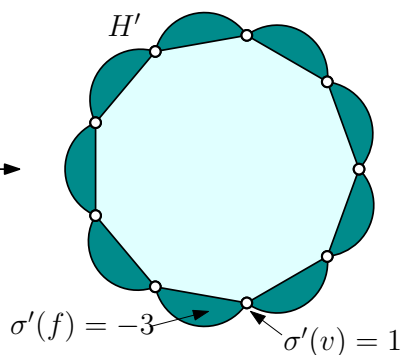

$\sigma^{\prime}(v)=1$
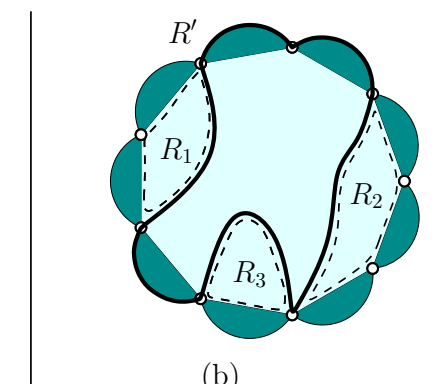

(b)

Figure 41. (a) The hypermap $H^{\prime}$ obtained from $H$ by adding a dark face of degree 2 along each outer edge. (b) The contour of a simply connected light region $R$ of $H^{\prime}$ containing the outer face $f_{0}^{\prime}$ but not all outer vertices. Here $\operatorname{deg}\left(f_{0}\right)=9, b=6, d=6$ and $k=3$

Claim 71. The charge function $\sigma^{\prime}$ fits $H^{\prime}$.

Proof. First observe that the charge $\sigma^{\prime}(v)$ of any vertex $v$ is positive, and $\sigma^{\prime}\left(f_{0}^{\prime}\right)=\operatorname{deg}\left(f_{0}^{\prime}\right)$. Moreover,

$$
\sigma_{\text {total }}^{\prime}=\sigma_{\text {total }}-\sigma\left(f_{0}\right)+\sigma^{\prime}\left(f_{0}^{\prime}\right)+\sum_{v \text { outer vertex }} \sigma^{\prime}(v)+\sum_{f \text { outer digon }} \sigma^{\prime}(f)=0
$$

because $\sigma_{\text {total }}=0,-\sigma\left(f_{0}\right)=\sigma^{\prime}\left(f_{0}^{\prime}\right)=\operatorname{deg}\left(f_{0}\right)$ and there are $\operatorname{deg}\left(f_{0}\right)$ outer vertices and outer digons.

We now prove that $H^{\prime}$ satisfies the $\sigma^{\prime}$-girth condition. Let $R$ be a light region of $H^{\prime}$. We want to prove $|\partial R| \geq \sigma^{\prime}(R)$ with strict inequality if an outer edge is strictly contained in $R$. By Lemma 32 we can assume that $R$ is simply connected. If $f_{0}^{\prime} \notin R$, then none of the outer digons is in $R$. Hence in this case $R$ is a light region of $H$, and $|\partial R| \geq \sigma(R)=\sigma^{\prime}(R)$ as wanted. We now assume that $f_{0}^{\prime} \in R$. First suppose that every outer vertex is strictly inside $R$. In this case, all the outer digons are in $R$, and we consider the light region $R$ of $H$ obtained from $R$ by replacing the outer face $f_{0}^{\prime}$ and the outer digons by $f_{0}$. We get $|\partial R|>\sigma(R)=\sigma^{\prime}(R)$ as wanted. Now assume that $f_{0}^{\prime} \in R$ but $b>0$ outer vertices are incident to $\partial R$ (so that $\operatorname{deg}\left(f_{0}\right)-b$ outer-vertices are strictly inside $R$ ). Let $d$ be the number of outer digons in $R$. By deleting from $R$ the outer face $f_{0}^{\prime}$ and the $d$ outer digons in $R$, one get a light region of $H$ which decomposes as a disjoint union of $k$ (nonempty) simply connected light regions $R_{1}, R_{2}, \ldots, R_{k}$; see Figure 41 (b). The number $k$ is determined by $k+\left(\operatorname{deg}\left(f_{0}\right)-d\right)=b$. Indeed the contour $D$ of the outer face of $f_{0}$ of $H$ decomposes into $b$ paths (joining consecutive vertices incident to $\partial R$ ) which are either 
edges of one of the $\operatorname{deg}\left(f_{0}\right)-d$ digons not in $R$, or part of the boundary of one of the light regions $R_{1}, R_{2}, \ldots, R_{k}$ (recall that $R$ is simply connected so that the light regions $R_{1}, \ldots, R_{k}$ corresponding to different paths of $D$ are distinct). We have

$$
|\partial R|=\sum_{i=1}^{k}\left|\partial R_{i}\right|+\operatorname{deg}\left(f_{0}\right)-2 d,
$$

and

$$
\sigma^{\prime}(R)=\sum_{i=1}^{k} \sigma\left(R_{i}\right)+\sigma^{\prime}\left(f_{0}^{\prime}\right)-3 d+\operatorname{deg}\left(f_{0}\right)-b=\sum_{i=1}^{k} \sigma\left(R_{i}\right)+2 \operatorname{deg}\left(f_{0}\right)-3 d-b,
$$

hence

$$
|\partial R|-\sigma^{\prime}(R)=\left(\sum_{i=1}^{k}\left|\partial R_{i}\right|-\sigma\left(R_{i}\right)\right)+b+d-\operatorname{deg}\left(f_{0}\right)=\left(\sum_{i=1}^{k}\left|\partial R_{i}\right|-\sigma\left(R_{i}\right)\right)+k \geq k .
$$

Thus, $|\partial R| \geq \sigma^{\prime}(R)$ and if one of the outer edges is strictly inside $R$, then $k \geq 1$ and $|\partial R|>\sigma^{\prime}(R)$

Since $\sigma^{\prime}$ fits $H^{\prime}$, Theorem 26 ensures that $H^{\prime}$ has a unique $\sigma^{\prime}$-weighted hyperorientation $\Omega^{\prime}$ in $\mathcal{H}_{+}$. Let $\Omega$ be the hyperorientation of $H$ such that the weights and orientations of the inner edges of $H$ are the same as in $\Omega^{\prime}$, and the outer edges of $H$ form a counterclockwise directed cycle of 1-way edges of weight 1.

Claim 72. The hyperorientation $\Omega$ is the unique $\sigma$-weighted hyperorientation of $H$ in $\mathcal{H}_{-}$.

Proof. Let $w^{\prime}(a)$ be the weight of a vertex, edge or face in $\Omega^{\prime}$. Let $D_{1}, \ldots, D_{\operatorname{deg}\left(f_{0}\right)}$ be the outer digons of $H^{\prime}$ in clockwise order, and let $e_{i}, e_{i}^{\prime}$ be the outer and inner edges incident to $D_{i}$ respectively. We will first prove that $w^{\prime}\left(e_{i}\right)=1$ and $w^{\prime}\left(e_{i}^{\prime}\right)=0$ for all $i \in\left\{1, \ldots, \operatorname{deg}\left(f_{0}\right)\right\}$. First note that for all $i \in\left\{1, \ldots, \operatorname{deg}\left(f_{0}\right)\right\}$, the weight condition on the outer digon $D_{i}$ gives $w^{\prime}\left(e_{i}\right)+w^{\prime}\left(e_{i}^{\prime}\right)=w^{\prime}\left(D_{i}\right)=-\sigma^{\prime}\left(D_{i}\right)-2=1$. Moreover, since the weight of every outer vertex $u$ is $w^{\prime}(u)=\sigma^{\prime}(u)=1$, we get $w^{\prime}\left(e_{i}\right) \leq 1$ and $w^{\prime}\left(e_{i}^{\prime}\right) \leq 1$. Hence $w^{\prime}\left(e_{i}\right) \geq 0$ and $w^{\prime}\left(e_{i}^{\prime}\right) \geq 0$, and $w^{\prime}\left(e_{i-1}\right)+w^{\prime}\left(e_{i}^{\prime}\right) \leq 1$ for all $i \in\left\{1, \ldots, \operatorname{deg}\left(f_{0}\right)\right\}$ with the convention that $e_{0}=e_{\operatorname{deg}\left(f_{0}\right)}$. Hence $w^{\prime}\left(e_{i-1}\right) \leq w^{\prime}\left(e_{i}\right)$ for all $i \in\left\{1, \ldots, \operatorname{deg}\left(f_{0}\right)\right\}$. Thus $w^{\prime}\left(e_{i-1}\right)=w^{\prime}\left(e_{i}\right)$ and $w^{\prime}\left(e_{i-1}^{\prime}\right)=w^{\prime}\left(e_{i}^{\prime}\right)$ for all $i \in\left\{1, \ldots, \operatorname{deg}\left(f_{0}\right)\right\}$. Moreover, the hyperorientation $\Omega^{\prime}$ has no counterclockwise directed cycle (since $\Omega^{\prime} \in \mathcal{H}_{+}$), hence $w^{\prime}\left(e_{i}^{\prime}\right)=0$ for all $i \in\left\{1, \ldots, \operatorname{deg}\left(f_{0}\right)\right\}$, and $w^{\prime}\left(e_{i}\right)=1$.

Since $w^{\prime}\left(e_{i}\right)=1$ and $w^{\prime}\left(e_{i}^{\prime}\right)=0$ for all $i \in\left\{1, \ldots, \operatorname{deg}\left(f_{0}\right)\right\}$, the weight of any vertex, or face of $H$ is the same in $\Omega$ as in $\Omega^{\prime}$. Moreover, the weight of every outer vertex and outer edge of $H$ in $\Omega$ is 1 . Thus $\Omega$ is $\sigma$-weighted. Moreover, because the hyperorientation $\Omega^{\prime}$ is minimal and accessible from the outer vertices, the hyperorientation $\Omega$ is also minimal and accessible from the outer vertices. Thus $\Omega$ is in $\mathcal{H}_{-}$.

Lastly, suppose there is another $\sigma$-weighted hyperorientation $\widetilde{\Omega} \neq \Omega$ of $H$ in $\mathcal{H}_{-}$. We then consider the hyperorientation $\widetilde{\Omega}^{\prime}$ of $H^{\prime}$ defined as follows: the weight of the inner edges of $H$ in $\widetilde{\Omega}^{\prime}$ are the same as in $\widetilde{\Omega}^{\prime}$, while the weight of the edges $e_{i}, e_{i}^{\prime}$ of the outer digon in $\widetilde{\Omega}^{\prime}$ are $\widetilde{w}^{\prime}\left(e_{i}\right)=1$ and $\widetilde{w}^{\prime}\left(e_{i}^{\prime}\right)=0$ for all $i \in\left\{1, \ldots, \operatorname{deg}\left(f_{0}\right)\right\}$. It is easily seen that $\widetilde{\Omega}^{\prime}$ is a $\sigma$-weighted hyperorientation of $H^{\prime}$ distinct from $\Omega^{\prime}$. Moreover $\widetilde{\Omega}^{\prime}$ is in $\mathcal{H}_{+}$(it is minimal, accessible from the outer vertices and the outer face of $H^{\prime}$ is a clockwise directed cycle). This contradicts the uniqueness of $\Omega^{\prime}$ given by Theorem 26 .

Claim 72 ensures that any dark-rooted charged hypermap $(H, \sigma)$ satisfying the conditions of Theorem 25 admits a unique $\sigma$-weighted hyperorientation in $\mathcal{H}_{-}$. This together with Lemma 56 completes the proof of Theorem 25.

We now prove Theorem 27 by a reduction to Theorem 25. Let $H$ be a vertex-rooted hypermap, and let $\sigma$ be a charge function fitting $H$. We want to prove that there exists a 
unique $\sigma$-weighted hyperorientation of $H$ in $\mathcal{H}_{0}$. Let $v_{0}$ be the root-vertex of $H$, and let $f_{0}$ be a light face incident to $v_{0}$. Let $H^{\prime}$ be the dark-rooted hypermap (with outer degree 1 ) obtained from $H$ by adding a loop edge $e_{0}$ incident to $v_{0}$ inside $f_{0}$ as indicated in Figure 42 The face of degree 1 incident to $e_{0}$ thus created (which is dark) is taken as the outer face of $H^{\prime}$, and is denoted by $f_{1}$. The light face of $H^{\prime}$ incident to $e_{0}$ is denoted by $f_{2}$. We define a charge function $\sigma^{\prime}$ of $H^{\prime}$ by setting $\sigma^{\prime}\left(f_{1}\right)=-1, \sigma^{\prime}\left(f_{2}\right)=\sigma\left(f_{0}\right)+1$ and $\sigma^{\prime}(a)=\sigma(a)$ for any other face or vertex of $H^{\prime}$.

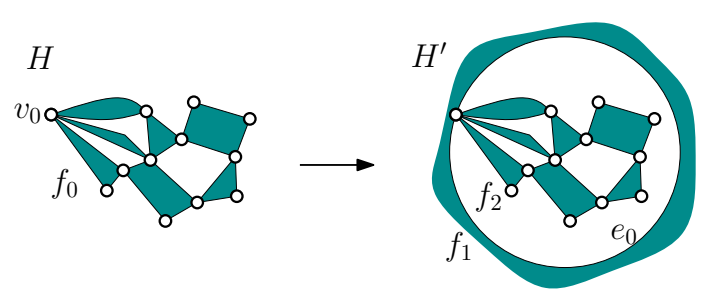

Figure 42. The dark-rooted hypermap $H^{\prime}$ obtained from $H$ by adding a loop edge $e_{0}$.

Claim 73. The charge function $\sigma^{\prime}$ fits the dark-rooted hypermap $H^{\prime}$.

Proof. Since $\sigma$ fits $H$, the charge $\sigma^{\prime}(v)$ of every inner vertex $v$ of $H^{\prime}$ is positive. Moreover the charge of the outer vertex $v_{0}$ is $\sigma^{\prime}\left(v_{0}\right)=\sigma\left(v_{0}\right)=0$, and the charge of the dark outer face $f_{1}$ is $\sigma^{\prime}\left(f_{1}\right)=-1=-\operatorname{deg}\left(f_{1}\right)$. Furthermore,

$$
\sigma_{\text {total }}^{\prime}=\sigma_{\text {total }}-\sigma\left(f_{0}\right)+\sigma^{\prime}\left(f_{1}\right)+\sigma^{\prime}\left(f_{2}\right)=0 .
$$

It remains to prove that $H^{\prime}$ satisfies the $\sigma^{\prime}$-girth condition. Let $R^{\prime}$ be a light region of $H^{\prime}$. First suppose that $f_{2} \notin R^{\prime}$. In this case $f_{1} \notin R^{\prime}$, hence $R^{\prime}$ is a light region of $H$ and $\left|\partial R^{\prime}\right| \geq \sigma\left(R^{\prime}\right)=\sigma^{\prime}\left(R^{\prime}\right)$ as wanted. Next suppose that both $f_{1}$ and $f_{2}$ are in $R^{\prime}$. In this case, we consider the light region $R$ of $H$ obtained from $R^{\prime}$ by replacing $f_{1}$ and $f_{2}$ by $f_{0}$. Since $\partial R=\partial R^{\prime}$, we get $\left|\partial R^{\prime}\right|=|\partial R| \geq \sigma(R)=\sigma^{\prime}\left(R^{\prime}\right)$ with strict inequality if $v_{0}$ is strictly inside $R^{\prime}$. Lastly, suppose that $f_{2} \in R^{\prime}$ and $f_{1} \notin R^{\prime}$. We consider the light region $R$ of $H$ obtained from $R^{\prime}$ by replacing $f_{2}$ by $f_{0}$. Note that $e_{0} \in \partial R^{\prime}$, and $\partial R=\partial R^{\prime} \backslash\left\{e_{0}\right\}$. Thus $\left|\partial R^{\prime}\right|=|\partial R|+1 \geq \sigma(R)+1=\sigma^{\prime}\left(R^{\prime}\right)$, as wanted.

Since $\sigma^{\prime}$ fits $H^{\prime}$, Theorem 25 implies that $H^{\prime}$ has a unique $\sigma^{\prime}$-weighted hyperorientation $\Omega^{\prime}$ in $\mathcal{H}_{-}$. Let $\Omega$ be the the restriction to $H$ of the hyperorientation $\Omega^{\prime}$.

Claim 74. The hyperorientation $\Omega$ is the unique $\sigma$-weighted hyperorientation of $H$ in $\mathcal{H}_{0}$.

Proof. By definition, the weight of $v_{0}$ and $e_{0}$ in $\Omega^{\prime}$ is 1 . Hence the weight of $v_{0}$ in $\Omega$ is 0 and the weight $w\left(f_{0}\right)$ of $f_{0}$ in $\Omega$ is the same as the weight $w^{\prime}\left(f_{2}\right)$ of $f_{2}$ in $\Omega^{\prime}$. Hence $w\left(f_{0}\right)=w^{\prime}\left(f_{2}\right)=\sigma^{\prime}\left(f_{2}\right)-\operatorname{deg}\left(f_{2}\right)=\sigma\left(f_{0}\right)-\operatorname{deg}\left(f_{0}\right)$. Hence the hyperorientation $\Omega$ is $\sigma$-weighted. Moreover because the hyperorientation $\Omega^{\prime}$ is minimal and accessible from $v_{0}$, the hyperorientation $\Omega$ is also minimal and accessible from $v_{0}$. Thus $\Omega$ is in $\mathcal{H}_{0}$.

Conversely, suppose that there is another $\sigma$-weighted hyperorientation $\widetilde{\Omega} \neq \Omega$ of $H$ in $\mathcal{H}_{0}$. We then consider the hyperorientation $\widetilde{\Omega}^{\prime}$ of $H^{\prime}$ defined as follows: the weight of $e_{0}$ is 1 and the weight of the other edges is as in $\widetilde{\Omega}$. It is easily seen that $\widetilde{\Omega}^{\prime}$ is a $\sigma$-weighted hyperorientation of $H^{\prime}$ distinct from $\Omega^{\prime}$. Moreover $\widetilde{\Omega}^{\prime}$ is in $\mathcal{H}_{-}$. This contradicts the uniqueness of $\Omega^{\prime}$ given by Theorem 25 .

Claim 74 shows that if a charge function $\sigma$ fits a vertex-rooted map $H$, then $H$ admits a unique $\sigma$-weighted hyperorientation in $\mathcal{H}_{0}$. This together with Lemma 56 completes the proof of Theorem 27

Acknowledgments. We thank the anonymous referees for their many useful comments and suggestions. 


\section{REFERENCES}

[1] M. Albenque and D. Poulalhon. A generic method for bijections between blossoming trees and planar maps. The Electronic Journal of Combinatorics, 22(2):P2-38, 2015.

[2] O. Bernardi. Bijective counting of tree-rooted maps and shuffles of parenthesis systems. Electron. J. Combin., 14(1):R9, 2007.

[3] O. Bernardi and G. Chapuy. A bijection for covered maps, or a shortcut between Harer-Zagier's and Jackson's formulas. J. Combin. Theory Ser. A, 118(6):1718-1748, 2011

[4] O. Bernardi and É. Fusy. A bijection for triangulations, quadrangulations, pentagulations, etc. J. Comb. Theory, Ser. A, 119(1):218-244, 2012.

[5] O. Bernardi and É. Fusy. Unified bijections for maps with prescribed degrees and girth. J. Combin. Theory Ser. A, 119(6):1351-1387, 2012.

[6] D.V. Boulatov and V. Kazakov. The Ising model on a random planar lattice: the structure of the phase transition and the exact critical exponents. Phys. Lett., B186(1):379-384, 1987.

[7] M. Bousquet-Mélou and G. Schaeffer. Enumeration of planar constellations. Adv. in Appl. Math., 24(4):337-368, 2000.

[8] M. Bousquet-Mélou and G. Schaeffer. The degree distribution of bipartite planar maps: applications to the Ising model. In K. Eriksson and S. Linusson, editors, Formal Power Series and Algebraic Combinatorics, pages 312-323, Vadstena, Sweden, 2003. ArXiv math.CO/0211070.

[9] J. Bouttier, P. Di Francesco, and E. Guitter. Census of planar maps: from the one-matrix model solution to a combinatorial proof. Nuclear Phys., B 645:477-499, 2002.

[10] J. Bouttier, P. Di Francesco, and E. Guitter. Planar maps as labeled mobiles. Electr. J. Combin., 11:R69, 2004.

[11] J. Bouttier, P. Di Francesco, and E. Guitter. Blocked edges on Eulerian maps and mobiles: Application to spanning trees, hard particles and the Ising model. J. Phys. A, 40:7411-7440, 2007.

[12] J. Bouttier and E. Guitter. Planar maps and continued fractions. Commun. Math., 309(3):623-662, 2012 .

[13] J. Bouttier and E. Guitter. On irreducible maps and slices, 2013. arXiv:1303.3728.

[14] U. Brandes and D. Wagner. A linear time algorithm for the arc disjoint Menger problem in planar directed graphs. Algorithmica, 28(1):16-36, 2000.

[15] É. Brézin, C. Itzykson, G. Parisi, and J.-B. Zuber. Planar diagrams. Comm. Math. Phys., 59:35-51, 1978.

[16] R. Cori and A. Machi. Maps, hypermaps and their automorphisms: a survey. Expo. Math., 10:403-467, 1992.

[17] R. Cori and B. Vauquelin. Planar maps are well labeled trees. Canad. J. Math., 33(5):1023-1042, 1981.

[18] E. Duchi, D. Poulalhon, and G. Schaeffer. Uniform random sampling of simple branched coverings of the sphere by itself. In Proceedings of the Twenty-Fifth Annual ACM-SIAM Symposium on Discrete Algorithms, SODA 2014, Portland, Oregon, USA, pages 294-304, 2014.

[19] S. Felsner. Lattice structures from planar graphs. Electron. J. Combin., 11(1), 2004.

[20] S. Felsner and K. Knauer. ULD-lattices and $\Delta$-bonds. Combinatorics, Probability and Computing, 18(5):707-724, 2009.

[21] É. Fusy, D. Poulalhon, and G. Schaeffer. Dissections, orientations, and trees, with applications to optimal mesh encoding and to random sampling. Transactions on Algorithms, 4(2):Art. 19, April 2008.

[22] I. Goulden and D. Jackson. Transitive factorisations into transpositions and holomorphic mappings on the sphere. Proceedings of the American Mathematical Society, 125(1):51-60, 1997.

[23] J. Hopcroft and R. Karp. An $n^{5 / 2}$ algorithm for maximum matchings in bipartite graphs. SIAM Journal on computing, 2(4):225-231, 1973.

[24] S. Khuller, J. Naor, and P. Klein. The lattice structure of flow in planar graphs. SIAM Journal on Discrete Mathematics, 6(3):477-490, 1993.

[25] S. K. Lando and A. K. Zvonkin. Graphs on Surfaces and Their Applications. Springer, 2004.

[26] J.-F. Le Gall. The topological structure of scaling limits of large planar maps. Inventiones Mathematica, 169:621-670, 2007.

[27] J.-F. Le Gall. Uniqueness and universality of the Brownian map. Ann. Probab., 41:2880-2960, 2013

[28] J.-F. Le Gall and T. Lehéricy. Separating cycles and isoperimetric inequalities in the uniform infinite planar quadrangulation. Annals of Probability, 2019. To appear.

[29] G. Miermont. On the sphericity of scaling limits of random planar quadrangulations. Elect. Comm. Probab., 13:248-257, 2008.

[30] G. Miermont. The Brownian map is the scaling limit of uniform random plane quadrangulations. Acta. Math., 210:319-401, 2013.

[31] A. Okounkov. Toda equations for Hurwitz numbers. Mathematical Research Letters, 7:447-453, 2000.

[32] D. Poulalhon and G. Schaeffer. A bijection for triangulations of a polygon with interior points and multiple edges. Theoret. Comput. Sci., 307(2):385-401, 2003. 
[33] D. Poulalhon and G. Schaeffer. Optimal coding and sampling of triangulations. Algorithmica, 46(34):505-527, 2006.

[34] J. Propp. Lattice structure for orientations of graphs. arXiv math/0209005, 2002.

[35] G. Schaeffer. Bijective census and random generation of Eulerian planar maps with prescribed vertex degrees. Electron. J. Combin., 4(1):\# 20, 14 pp., 1997.

[36] G. Schaeffer. Conjugaison d'arbres et cartes combinatoires aléatoires. PhD thesis, Université Bordeaux I, 1998.

[37] A. Schrijver. Combinatorial Optimization. Springer, 2003.

[38] W. T. Tutte. A census of planar maps. Canad. J. Math., 15:249-271, 1963. 